\title{
Micro-to-macro transition accounting for general imperfect interfaces
}

\author{
Ali Javili $^{\mathrm{a}, *}$, Paul Steinmann ${ }^{\mathrm{b}}$, Jörn Mosler ${ }^{\mathrm{c}}$ \\ ${ }^{a}$ Department of Mechanical Engineering, Bilkent University, 06800 Ankara, Turkey \\ ${ }^{\mathrm{b}}$ Chair of Applied Mechanics, University of Erlangen-Nuremberg, Egerlandstr. 5, 91058 Erlangen, Germany \\ ${ }^{\mathrm{c}}$ Institute of Mechanics, TU Dortmund, Leonhard-Euler-Str. 5, 44227-Dortmund, Germany
}

Received 11 May 2016; received in revised form 8 December 2016; accepted 15 December 2016

Available online 21 December 2016

\section{Highlights}

- To establish a computational homogenization scheme accounting for interfaces at the microscale.

- To present a thermodynamically consistent formulation and governing equations of general imperfect interfaces.

- To provide a suitable finite element framework for numerical implementation of continua with generalized interfaces.

- To elucidate the theory via a series of numerical examples.

- To capture the smaller-stronger and smaller-weaker size effect on the overall material response.

\begin{abstract}
The objective of this contribution is to establish a micro-to-macro transition framework to study the behavior of heterogeneous materials whereby the influence of interfaces at the microscale is taken into account. The term "interface" refers to a zero-thickness model that represents the finite thickness "interphase" between the constituents of the micro-structure. For geometrically equivalent samples, due to increasing area-to-volume ratio with decreasing size, interfaces demonstrate a more pronounced effect on the material response at small scales. A remarkable outcome is that including interfaces introduces a length-scale and our interfaceenhanced computational homogenization captures a size effect in the material response even if linear prolongation conditions are considered. Furthermore, the interface model in this contribution is general imperfect in the sense that it allows for both jumps of the deformation as well as for the traction across the interface. Both cohesive zone model and interface elasticity theory can be derived as two limit cases of this general model. We establish a consistent computational homogenization scheme accounting for general imperfect interfaces. Suitable boundary conditions to guarantee meaningful averages are derived. Clearly, this general framework reduces to classical computational homogenization if the effect of interfaces is ignored. Finally, the proposed theory is elucidated via a series of numerical examples.
\end{abstract}

(C) 2016 Elsevier B.V. All rights reserved.

Keywords: General imperfect interface; Cohesive zone; Interface elasticity; Computational homogenization; Size effect; Nano-materials

\footnotetext{
* Corresponding author.

E-mail addresses: ajavili@bilkent.edu.tr (A. Javili), paul.steinmann@1tm.uni-erlangen.de (P. Steinmann), joern.mosler@tu-dortmund.de (J. Mosler).
} 


\section{Introduction}

Effective macroscopic properties of a heterogeneous material can be estimated from the response of its underlying micro-structure using homogenization procedures. These mature procedures need to be extended to account for the role of interfaces between the constituents at the microscale and consequently to capture the size-effect missing in classical computational homogenization schemes. The objective of this contribution is to present a novel micro-tomacro transition (computational homogenization) framework that accounts for interfaces at the microscale. Thus, the two main ingredients of the work presented here are (i) to formulate a general imperfect interface model and (ii) to extend the homogenization theory as pioneered by Hill [1,2]. A brief review of these topics is now given in Sections 1.1 and 1.2.

\subsection{State of the art review of interface models}

The term interface essentially corresponds to a zero-thickness model representing the finite thickness interphase between different bulk phases. From this viewpoint, an interface is a two-dimensional manifold in the threedimensional embedding space and therefore, the interface energy over the bulk energy reads

$$
\frac{\text { interface energy }}{\text { bulk energy }}=\frac{\text { interface energy density } \times \text { area }}{\text { bulk energy density } \times \text { volume }}=\frac{\text { interface energy density }}{\text { bulk energy density }} \times \frac{\text { area }}{\text { volume }} .
$$

The ratio of the interface energy over the bulk energy is a decisive measure to indicate whether or not interface effects are negligible. While the ratio of the energy densities depends solely on the material, the area-to-volume ratio is proportional to the inverse of the problem dimension. Therefore, for geometrically equivalent samples, the interface effects become more important at smaller scales and this fact has been the main motivation to develop different interface models in the past. Interface models can be subdivided into different classes based on continuity of certain variables associated with the problem. Within the context of mechanical problems, the displacement and the traction play the main role to categorize interface models. Fig. 1 summarizes schematically various possible interface models in the context of mechanical problems briefly reviewed in what follows.

\section{- Perfect interface model}

The first family of interfaces are trivial interfaces categorized as perfect interface model, also referred to as free singular surfaces [3]. This interface model does not have any sophisticated characteristic and is merely defined to better understand other interface models. Both the displacement and the traction are continuous across a perfect interface.

\section{- Cohesive interface model}

If either the displacement or the traction assumes a jump across the interface, the interface model is imperfect. Cohesive interface model is one subset of imperfect interfaces and allows for displacement jumps across the interface but continuity of the traction is central to such interface models. The cohesive interface model is based on the classical cohesive zone model and dates back to the seminal works of Barenblatt [4,5] and Dugdale [6] and has been extensively studied (see [7-10], among others) with applications to debonding, decohesion and fracture from both theoretical and computational aspects. For recent advances on cohesive zone models as well as improved traction-separation laws to describe the interface behavior, see [11-18] and references therein.

\section{- Elastic interface model}

In contrast to the cohesive interface model, the displacement is continuous across an elastic interface but the traction is discontinuous, in general. The jump of the traction in the elastic interface model is associated with the tension along the interface as well as the curvature of the interface. The interface elasticity theory [19-22] roots in the surface elasticity theory of Gurtin and Murdoch [23] and has been further extended in [24-26] to account for the curvature-dependence of the surface free-energy density. Such interfaces fall into the category of thermodynamic singular surfaces [3] whose balance equation reduces to the generalized Young-Laplace equation [27]. For further details on interface elasticity see $[28,29]$ and references therein.

\section{- General interface model}

Based on the classification above, it is clear that both cohesive and elastic interface models are only two extremes of all possible responses allowing both jumps in the displacement and the traction termed altogether as general interface model. While both isotropic cohesive and elastic interface models are well-established to date, the 
perfect interface model

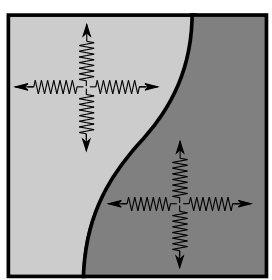

displacement jump $=0$ traction jump $=0$ cohesive interface model

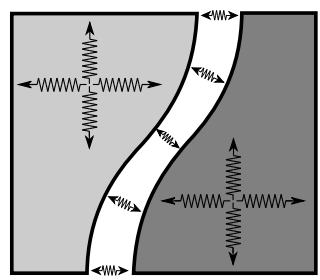

displacement jump $\neq 0$ traction jump $=0$ elastic interface model

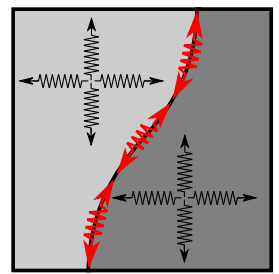

displacement jump $=0$ traction jump $\neq 0$ general interface model

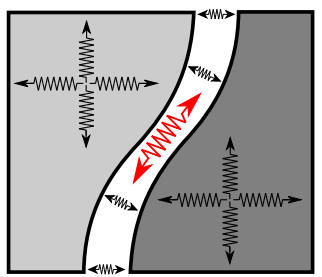

displacement jump $\neq 0$ traction jump $\neq 0$

Fig. 1. Classification of interface models. The perfect interface model does not allow for the displacement jump nor the traction jump across the interface. If either of the displacement jump or the traction jump across the interface is non-vanishing, the interface model is referred to as imperfect. The imperfect interface models are cohesive interface model, elastic interface or the combination of the both termed general imperfect interface model or simply, general interface model.

general interface model is poorly understood and barely studied with the exception of some analytical works e.g. [30-34] mainly limited to small strains and derived from asymptotic expansions with certain simplifying assumptions necessary for the analytical approach. ${ }^{1}$ Only very recently, Ottosen et al. [35] extended the work of Steinmann and Häsner [36] from the small strain to the finite deformation setting and mathematically formulated non-coherent interfaces possessing an elastic resistance along the interface. Our description of the general interface model is valid in the finite deformation setting and furthermore both theoretical and numerical aspects are thoroughly investigated. In the context of thermal problems this concept of general interfaces exists [37,38] and dates back to Hashin [39] or perhaps to Sanchez-Palencia [40] whereby the heat conduction along the interface resembles the interface elasticity in the mechanical problem. In fact, this work may be viewed as the mechanical counterpart of our recent contribution [38] where only the thermal problem was considered.

\subsection{State of the art review of computational homogenization}

The overall behavior of composite materials depends on their underlying micro-structure such as volume fraction, shape, distribution and orientation of their constituents at the microscale. Therefore, it is challenging to predict the macroscopic material response based on the information at the microscale and this task requires sophisticated techniques such as homogenization. The foundations of homogenization were laid down by Hill [1,2] and Ogden [41] which are closely related to the works of Eshelby [42] and Hashin [43,44]. For further details on analytical homogenization, see [45-51] and references therein. Homogenization is a powerful methodology to link microscopic and macroscopic scales and provides the basis for computational micro-to-macro transition [52]. Computational homogenization is a mature field and has been broadly studied in the past two decades [53-68] and thoroughly reviewed in [69-73].

A major controversy with the classical (first-order) computational homogenization is that it cannot account for the size dependent behavior of materials frequently referred to as the size effect. Kouznetsova et al. [74] have circumvented this issue by incorporating higher gradients into the material response and hence, proposed secondorder computational homogenization. ${ }^{2}$ Here, we capture a size effect via accounting for interfaces at the microscale. This approach is intuitive due to the increasing area-to-volume fraction at the microscale. Numerous analytical works e.g. [88-90] as well as numerical contributions e.g. [91-93] have studied the size effect as a result of the surface elastic response motivated by the surface elasticity theory. Comparisons with atomistic simulations have shown that such surface-driven size effects are physically meaningful [94-97]. For the importance of surface effects versus secondgradient effects in size-dependent behavior of nano-objects, see [98]. In analogy to elastic interfaces, Leuschner and Fritzen [99] recently have incorporated the cohesive interface model in the microscale, see also [100].

\footnotetext{
${ }^{1}$ In that "analytical community" of formulating the interface as the asymptotic limit of a thin interphase, the cohesive interface model and elastic interface model are often referred to as spring interface model and stress interface model, respectively, as the two limit cases of soft or stiff interphase, respectively [see for instance [33] among others].

2 For higher-gradient elasticity theories, see for instance [75-87] among others.
} 
Although both extreme limits of general interfaces namely, elastic interfaces as well as cohesive interfaces at the micro-structure have been investigated in the past, a unified framework to account for the general imperfect interface model is missing until now and this subject shapes the structure of this manuscript. We extend the computational homogenization framework in this contribution to account for general imperfect interfaces. It is shown that in contrast to a purely cohesive or purely elastic interface model that results in a uniform size dependent response, the general imperfect interfaces lead to a non-monotonic and complex size effect. In passing we mention that the general imperfect interface model here simplifies to the surface elasticity in the limit where the inclusion becomes extremely compliant to the matrix. Thus, free surfaces can be understood as the limit case of solid-solid interfaces presented here.

\subsection{Organization of this manuscript}

This manuscript is organized as follows. Notation and definitions are shortly introduced. Section 2 deals with theoretical aspects of computational homogenization at finite strains accounting for general imperfect interfaces. First, the theory of general imperfect interfaces and governing equations are derived in Section 2.1. Next, in Section 2.2 a consistent framework for the micro-to-macro transition is introduced that can account for interfaces. The balance equations at the macroscale and the microscale are briefly introduced and meaningful averaging theorems are proposed to link the two scales. Clearly, the modified averaging theorems reduce to the classical ones in the absence of interfaces. The classical Hill-Mandel condition is extended to establish an incremental energy equivalence between the scales. Suitable boundary conditions are derived such that the Hill-Mandel condition is a priori satisfied. Section 4 elucidates the developed theory by a series of numerical examples using the finite element method. Sections 5 and 6 concludes this work and discusses possible extensions and outlooks.

\section{Notation and definitions}

At the microscale, quantities defined on the interface are distinguished from those in the bulk material by a bar placed above the quantity. That is, $\{\bar{\bullet}\}$ refers to an interface variable with its bulk counterpart being $\{\bullet\}$. Following this convention throughout the manuscript, surface, interface and curve quantities are denoted as $\{\hat{\boldsymbol{\bullet}}\},\{\overline{\boldsymbol{\bullet}}\}$ and $\{\tilde{\boldsymbol{\bullet}}\}$, respectively and therefore distinguished from the bulk quantity $\{\bullet\}$ by an accent on top of the quantity. Moreover, macroscopic quantities are differentiated from microscale quantities by the left super-script " $M$ " placed next to the quantity. That is, $\mathrm{M}_{\{\bullet\}}$ refers to a macroscopic variable with its microscopic counterpart being $\{\bullet\}$. The terms "macro" and "micro" are frequently used instead of macroscopic and microscopic, respectively.

Within the classical homogenization context, the term "size" usually refers to the size of the window zoomed at the material micro-structure and when this window is large enough such that apparent macroscopic properties converge, then this window serves as a representative volume element abbreviated as RVE, see for instance [101] for further details on the definition of the RVE. Classical first-order computational homogenization is missing a length-scale or physical size of the RVE and therefore, the size defined as such does not introduce any confusion in that context. This is not the case here. In particular, we consider perfectly periodic micro-structures such that the unit cell is by definition representative and hence a RVE. In the discussions of the numerical results varying the RVE-size indicates varying the physical size or the dimension of the RVE.

Direct notation is adopted throughout. Occasional use is made of the index notation, the summation convention for repeated indices being implied. The three-dimensional Euclidean space is denoted $\mathbb{E}^{3}$. The second-order identity tensor is denoted $\boldsymbol{i}$ or $\boldsymbol{I}$ in the spatial and material configurations, respectively, and are identical. The dyadic product of two vectors $\boldsymbol{a}$ and $\boldsymbol{b}$ is a second-order tensor $\boldsymbol{D}=\boldsymbol{a} \otimes \boldsymbol{b}$ with $[\boldsymbol{D}]_{i j}=[\boldsymbol{a}]_{i}[\boldsymbol{b}]_{j}$. The scalar product of two vectors $\boldsymbol{a}$ and $\boldsymbol{b}$ is denoted $\boldsymbol{a} \cdot \boldsymbol{b}=[\boldsymbol{a}]^{i}[\boldsymbol{b}]_{i}=[\boldsymbol{a} \otimes \boldsymbol{b}]: \boldsymbol{i}$ in which the scalar product of two second-order tensors $\boldsymbol{A}$ and $\boldsymbol{B}$ is denoted $\boldsymbol{A}: \boldsymbol{B}=[\boldsymbol{A}]^{i j}[\boldsymbol{B}]_{i j}$. The composition of two second-order tensors $\boldsymbol{A}$ and $\boldsymbol{B}$, denoted $\boldsymbol{A} \cdot \boldsymbol{B}$, is a second-order tensor with components $[\boldsymbol{A} \cdot \boldsymbol{B}]_{i j}=[\boldsymbol{A}]_{i}^{m}[\boldsymbol{B}]_{m j}$. The vector product of two vectors $\boldsymbol{a}$ and $\boldsymbol{b}$ is denoted $\boldsymbol{a} \times \boldsymbol{b}$ with $[\boldsymbol{a} \times \boldsymbol{b}]_{k}=\boldsymbol{\varepsilon}:[\boldsymbol{a} \otimes \boldsymbol{b}]=[\boldsymbol{\varepsilon}]_{i j k}[\boldsymbol{a}]^{i}[\boldsymbol{b}]^{j}$ where $\varepsilon$ denotes the permutation (LeviCivita) tensor. The action of a second-order tensor $\boldsymbol{A}$ on a vector $\boldsymbol{a}$ is given by $[\boldsymbol{A} \cdot \boldsymbol{a}]_{i}=[\boldsymbol{A}]_{i}^{j}[\boldsymbol{a}]_{j}$. The nonstandard dot product of a fourth-order tensor $\mathbb{C}$ on a vector $\boldsymbol{a}$ is given by $[\boldsymbol{a}: \mathbb{C}]_{i j k}=[\mathbb{C}]_{i s j k}[\boldsymbol{a}]^{s}$. Two non-standard dyadic products of two second-order tensors $\boldsymbol{A}$ and $\boldsymbol{B}$ are the fourth-order tensors $[\boldsymbol{A} \bar{\otimes} \boldsymbol{B}]_{i j k l}=[\boldsymbol{A}]_{i k}[\boldsymbol{B}]_{j l}$ and $[\boldsymbol{A} \otimes \boldsymbol{B}]_{i j k l}=[\boldsymbol{A}]_{i l}[\boldsymbol{B}]_{j k}$. The non-standard dyadic product between a vector $\boldsymbol{a}$ and a second-order tensors $\boldsymbol{B}$ is a third-order tensor $[\boldsymbol{a} \bar{\otimes} \boldsymbol{B}]_{i j k}=[\boldsymbol{B} \bar{\otimes} \boldsymbol{a}]_{i j k}=[\boldsymbol{a}]_{j}[\boldsymbol{B}]_{i k}=\boldsymbol{a} \cdot[\boldsymbol{i} \bar{\otimes} \boldsymbol{B}]$. The average and jump of a quantity $\{\bullet\}$ over 

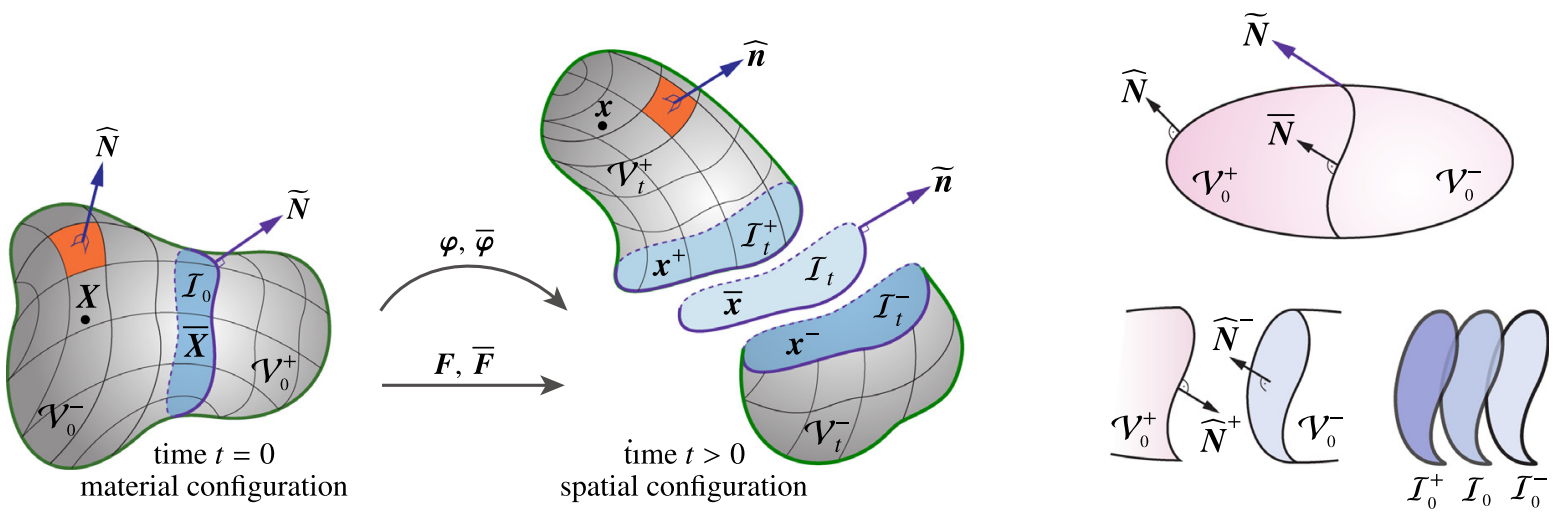

Fig. 2. The material and spatial configuration of a cutout volume of a continuum body and its associated motion and deformation gradient. The two sides of the body in the material configuration, $\mathcal{V}_{0}^{-}$and $\mathcal{V}_{0}^{+}$, are bonded via the interface $\mathcal{I}_{0}$. The interface initially has no thickness and is only a sharp surface connecting the two sides of the bulk, i.e. $\llbracket X \rrbracket=\mathbf{0}$. Through the motion $\boldsymbol{\varphi}$ the interface opens resulting in the displacement jump $\llbracket \boldsymbol{x} \rrbracket \neq \mathbf{0}$ across the interface.

the interface are defined by $\{\{\bullet \bullet\}\}=\frac{1}{2}\left[\{\bullet\}^{+}+\{\bullet\}^{-}\right]$and $\mathbb{\{}\{\bullet\} \|=\{\bullet\}^{+}-\{\bullet\}^{-}$, respectively. The average and jump operators show the property $\mathbb{L}\{\bullet\} \cdot\{0\} \rrbracket=\mathbb{L}\{\bullet\} \rrbracket \cdot\{\{0\}\}+\{\{\{\bullet\}\} \cdot \mathbb{[}\{0\} \rrbracket$.

\section{Theory}

The objective of this section is to establish the governing equations of a heterogeneous material within the framework of continuum mechanics at finite deformation. In particular, we focus on the influence of the interface between the constituents and its implications. In doing so, the problem is decomposed into two different length scales namely, the macroscopic length scale ${ }^{\mathrm{M}_{\ell}}$ and the microscopic lengths scale $\ell \ll \mathrm{M}_{\ell}$. This separation of length scales has two outcomes. First, the unknown material behavior at the macroscale can be computed via homogenizing the response of the underlying micro-structure for which the constitutive laws are assumed to be known. Second, the influence of interfaces on the overall response at the microscale can no longer be neglected due to the large area-tovolume ratio. The two main ingredients of this section are therefore: formulation of general imperfect interface models presented in Section 2.1 and to properly incorporate such interfaces into a computational homogenization framework detailed in Section 2.2. In this contribution, all relations and derivations correspond to the Lagrangian description, keeping in mind that it is straightforward to re-formulate the problem in the Eulerian framework. Recall that the primary objective of this work is to account for interfaces within a computational homogenization scheme. Therefore, we limit the discussion to major relations and definitions that are essential for the paper to be self-contained. Further details on computational homogenization and its underlying assumptions as well as the interface models available in the literature can be found in the extensive references listed in the introduction.

\subsection{General imperfect interfaces}

The purpose of this section is to summarize certain key concepts in continuum mechanics and to introduce the theory of general imperfect interfaces in the context of a mechanical problem. Detailed expositions on nonlinear continuum mechanics can be found in [102-104] among others. Basic concepts and terminologies corresponding to the fundamentals of differential geometry on surfaces are briefly reviewed in Appendix A.

\subsubsection{Problem definition}

Consider a continuum body that takes the material configuration $\mathcal{V}_{0}$ at time $t=0$ and the spatial configuration $\mathcal{V}_{t}$ at any time $t$ as shown in the Fig. 2. The configurations $\mathcal{V}_{0}$ and $\mathcal{V}_{t}$, consistent with Section 2.2, correspond to an arbitrary cutout volume of a body. Note, we restrict the analysis only to quasi-static conditions and thus, time plays the role of a history parameter to order the sequence of events.

The reference placement of material particles in the bulk and on the interface are labeled $\boldsymbol{X}$ and $\overline{\boldsymbol{X}}$, respectively. The motion from the material to the spatial configuration in the bulk and on the interface is denoted as $\varphi$ and $\bar{\varphi}$, respectively. 
The current placement of material particles in the bulk and on the interface are labeled $\boldsymbol{x}$ and $\overline{\boldsymbol{x}}$, respectively. The linear deformation maps associated with $\boldsymbol{\varphi}$ and $\overline{\boldsymbol{\varphi}}$ are denoted $\boldsymbol{F}$ and $\overline{\boldsymbol{F}}$, respectively and relate the infinitesimal line elements from the material to the spatial configuration as

$$
\boldsymbol{x}=\boldsymbol{\varphi}(\boldsymbol{X}) \quad \Rightarrow \quad \mathrm{d} \boldsymbol{x}=\boldsymbol{F} \cdot \mathrm{d} \boldsymbol{X}, \quad \overline{\boldsymbol{x}}=\overline{\boldsymbol{\varphi}}(\overline{\boldsymbol{X}}) \quad \Rightarrow \quad \mathrm{d} \overline{\boldsymbol{x}}=\overline{\boldsymbol{F}} \cdot \mathrm{d} \overline{\boldsymbol{X}},
$$

in which $\boldsymbol{F}=\operatorname{Grad} \boldsymbol{\varphi}$ and $\overline{\boldsymbol{F}}=\overline{\operatorname{Grad}} \overline{\boldsymbol{\varphi}}=\boldsymbol{F} \cdot \overline{\boldsymbol{I}}$ with $\overline{\boldsymbol{I}}$ being the interface identity tensor in the material configuration defined by $\overline{\boldsymbol{I}}=\boldsymbol{I}-\overline{\boldsymbol{N}} \otimes \overline{\boldsymbol{N}}$ with $\overline{\boldsymbol{N}}$ being the interface unit normal. Analogously, the interface identity tensor in the spatial configuration is defined by $\overline{\boldsymbol{i}}=\boldsymbol{i}-\overline{\boldsymbol{n}} \otimes \overline{\boldsymbol{n}}$ with $\overline{\boldsymbol{n}}$ being the interface unit normal in the spatial configuration. Let $\mathrm{d} V$ and $\mathrm{d} v$ denote the volume elements of the bulk in the material and spatial configurations, respectively and similarly, $\mathrm{d} A$ and $\mathrm{d} a$ denote the area elements of the interface in the material and spatial configurations, respectively. The ratios of volume elements and area elements in the spatial over the material configuration are denoted $J$ and $\bar{J}$, respectively, as

$$
J=\mathrm{d} v / \mathrm{d} V \quad \text { with } \quad J:=\operatorname{Det} \boldsymbol{F} \quad \text { and } \quad \bar{J}=\mathrm{d} a / \mathrm{d} A \quad \text { with } \quad \bar{J}:=\overline{\operatorname{Det}} \overline{\boldsymbol{F}} .
$$

The configuration $\mathcal{V}_{0}$ consists of two disjoint subdomains denoted as open sets $\mathcal{V}_{0}^{-}$and $\mathcal{V}_{0}^{+}$with the interface $\mathcal{I}_{0}$ in between and therefore, $\mathcal{V}_{0}=\mathcal{V}_{0}^{+} \cup \mathcal{I}_{0} \cup \mathcal{V}_{0}^{-}$. The intersection of the interface $\mathcal{I}_{0}$ with the boundary of each subdomain defines the two sides of the interface as $\mathcal{I}_{0}^{-}=\partial \mathcal{V}_{0}^{-} \cap \mathcal{I}_{0}$ and $\mathcal{I}_{0}^{+}=\partial \mathcal{V}_{0}^{+} \cap \mathcal{I}_{0}$. In the material configuration, the interface coincides geometrically with its sides $\mathcal{I}_{0}=\mathcal{I}_{0}^{-}=\mathcal{I}_{0}^{+}$. Let $\boldsymbol{X}^{-}$and $\boldsymbol{X}^{+}$denote the particles on $\mathcal{I}_{0}^{-}$and $\mathcal{I}_{0}^{+}$, respectively. Similarly, $\boldsymbol{x}^{-}$and $\boldsymbol{x}^{+}$denote the particles on $\mathcal{I}_{t}^{-}$and $\mathcal{I}_{t}^{+}$, respectively, in the spatial configuration. The interface motion $\bar{\varphi}$ defines the interface in the spatial configuration $\mathcal{I}_{t}$ with its particles labeled $\bar{x}$. However, the two sides of the interface map via the bulk motion $\varphi$ resulting in a geometrical jump in the spatial configuration as

$$
\bar{x}=\bar{\varphi}(\bar{X}), \quad \bar{x} \in \mathcal{I}_{t}, \quad x^{-}=\varphi\left(X^{-}\right), \quad x^{-} \in \mathcal{I}_{t}^{-}, \quad x^{+}=\varphi\left(X^{+}\right), \quad x^{+} \in \mathcal{I}_{t}^{+} .
$$

It is of particular importance to note that so far, we did not impose any dependence of the interface motion $\bar{\varphi}$ on the bulk motion $\varphi$. Also, since the two sides of the interface coincide in the material configuration, the spatial geometrical jump at the interface $\llbracket x \rrbracket$ is purely related to the bulk motion jump $\llbracket \varphi \rrbracket$.

In the material configuration, the outward unit normal vector to the boundary of the body $\partial \mathcal{V}_{0}$ is denoted by $\widehat{N}$. Recall, the quantities with a hat are surface quantities and the unit normal $\widehat{N}$ is essentially a surface normal. The normal to the interface $\mathcal{I}_{0}$ is denoted $\bar{N}$ and points from the minus side $\mathcal{I}_{0}^{-}$to the plus side $\mathcal{I}_{0}^{+}$of the interface. The normals to the minus and plus sides of the interface are denoted $\widehat{N}^{-}$and $\widehat{N}^{+}$, respectively. The normal to the boundary of the interface $\partial \mathcal{I}_{0}$ but, tangential to the interface $\mathcal{I}_{0}$ is denoted $\widetilde{N}$. Note, the interface boundary $\partial \mathcal{I}_{0}$ is a curve whose normal or bi-normal, in the sense of the Frénet-Serret formula, do not necessarily coincide with $\widetilde{N}$. Also, $\widetilde{N}$ is not necessarily normal to the surface as shown in Fig. 2. In the spatial configuration, the surface, interface and curve normals are denoted $\widehat{\boldsymbol{n}}, \overline{\boldsymbol{n}}$ and $\widetilde{\boldsymbol{n}}$, respectively.

\subsubsection{Governing equations}

The governing equations of the problem can be categorized as balance equations and constitutive laws. Balance equations refer to mechanical balance equations, i.e. the balance of linear and angular momentum. Constitutive laws are the results of thermodynamics considerations and consistencies. Here, we first derive the balance equations and then, following thermodynamics arguments, we propose thermodynamically consistent constitutive laws and evolutions for the interface. To derive the governing equations, we adopt a standard procedure [104] carefully revised to account for the general interfaces.

Balance equations. The balance equations are derived by viewing the configuration $\mathcal{V}_{0}$ as an arbitrary (canonical) subdomain or an arbitrary cutout volume of a continuum body. This view is only assumed to reduce the notations and it does not alter the derivations nor the final equations. The procedure consists of three main steps. First, we write the global mechanical power or the external working ${ }^{3} \mathcal{W}_{0}$ on the arbitrary cutout volume $\mathcal{V}_{0}$ in an integral form. Second, we impose the invariance of working $\mathcal{W}_{0}$ with respect to superposed rigid body motion. The invariance of $\mathcal{W}_{0}$ with

\footnotetext{
${ }^{3}$ Following Gurtin [105], the term working is used consistently throughout the manuscript alternative to the more intuitive expression external mechanical power and is the rate of the work due to external forces.
} 
respect to translation results in the balance of linear momentum and the invariance of $\mathcal{W}_{0}$ with respect to rotation renders the balance of angular momentum. The obtained balances of linear and angular momentum are in global (integral) form. Third and finally, we derive the local balance equations by localizing the global balances. In doing so, we utilize the arbitrariness of the cutout volume and set the integral domain to a vanishing volume in the limit.

The global working or the (external) mechanical power in the material configuration denoted as $\mathcal{W}_{0}$ reads

$$
\begin{aligned}
\mathcal{W}_{0}= & \mathcal{W}_{0}(\dot{\boldsymbol{\varphi}}, \dot{\overline{\boldsymbol{\varphi}}})=\int_{\mathcal{V}_{0}^{-}} \dot{\boldsymbol{\varphi}} \cdot \boldsymbol{b}_{0} \mathrm{~d} V+\int_{\mathcal{V}_{0}^{+}} \dot{\boldsymbol{\varphi}} \cdot \boldsymbol{b}_{0} \mathrm{~d} V+\int_{\partial \mathcal{V}_{0}^{-}} \dot{\boldsymbol{\varphi}} \cdot \widehat{\boldsymbol{b}}_{0} \mathrm{~d} A \\
& +\int_{\partial \mathcal{V}_{0}^{+}} \dot{\boldsymbol{\varphi}} \cdot \widehat{\boldsymbol{b}}_{0} \mathrm{~d} A+\int_{\mathcal{I}_{0}} \dot{\overline{\boldsymbol{\varphi}}} \cdot \overline{\boldsymbol{b}}_{0} \mathrm{~d} A+\int_{\partial \mathcal{I}_{0}} \dot{\overline{\boldsymbol{\varphi}}} \cdot \widetilde{\boldsymbol{b}}_{0} \mathrm{~d} L,
\end{aligned}
$$

in which $\dot{\varphi}$ and $\dot{\bar{\varphi}}$ denote the material time derivatives of the bulk and interface motion $\varphi$ and $\bar{\varphi}$, respectively. Note, the boundaries $\partial \mathcal{V}_{0}^{-}$and $\partial \mathcal{V}_{0}^{+}$assume the same motion as the bulk itself in the sense of kinematic slavery. In other words, the surface always remains on the boundary and neither detaches from the bulk nor penetrates the bulk and therefore, the surface is material. The same analogy holds for the boundary of the interface namely, the curve $\partial \mathcal{I}_{0}$ which assumes the same motion $\bar{\varphi}$ and the same velocity $\dot{\bar{\varphi}}$ as the interface. The force density of the bulk per unit reference volume in the material configuration is denoted $\boldsymbol{b}_{0}$. Similarly, the force density of the surface per unit reference area in the material configuration is denoted $\widehat{\boldsymbol{b}}_{0}$, often referred to as (surface) traction. In a near identical fashion, the force density of the curve per unit reference length in the material configuration is denoted $\widetilde{\boldsymbol{b}}_{0}$. For the sake of completeness, we allow a force density on the interface per unit reference area in the material configuration and denote it as $\overline{\boldsymbol{b}}_{0}$.

Following Cauchy theorem type arguments, the surface traction $\widehat{\boldsymbol{b}}_{0}$ can be related to the stresses in the material $\boldsymbol{P}$ through the surface normal $\widehat{N}$ according to $\widehat{\boldsymbol{b}}_{0}=\boldsymbol{P} \cdot \widehat{\boldsymbol{N}}$. Interface elasticity theory [19] and its sibling surface elasticity theory [23] are based on Cauchy theorem type arguments for a two-dimensional manifold. In this view, the interface is endowed with its own stress $\overline{\boldsymbol{P}}$ and the traction $\widetilde{\boldsymbol{b}}_{0}$ on the boundary of the interface $\partial \mathcal{I}_{0}$ is related to the interface stress via $\widetilde{\boldsymbol{b}}_{0}=\overline{\boldsymbol{P}} \cdot \widetilde{\boldsymbol{N}}$. The interface stress $\overline{\boldsymbol{P}}$ is superficial in the sense that it possesses the property $\overline{\boldsymbol{P}} \cdot \overline{\boldsymbol{N}}=\mathbf{0}$. The superficiality of the interface stress is a crucial assumption in this context. Javili et al. [106] have shown that the superficiality property is the consequence of a first-order continuum theory. Rewriting Eq. (4) in terms of stresses instead of tractions yields

$$
\begin{aligned}
\mathcal{W}_{0}= & \mathcal{W}_{0}(\dot{\boldsymbol{\varphi}}, \dot{\overline{\boldsymbol{\varphi}}})=\int_{\mathcal{V}_{0}^{-}} \dot{\boldsymbol{\varphi}} \cdot \boldsymbol{b}_{0} \mathrm{~d} V+\int_{\mathcal{V}_{0}^{+}} \dot{\boldsymbol{\varphi}} \cdot \boldsymbol{b}_{0} \mathrm{~d} V+\int_{\partial \mathcal{V}_{0}^{-}} \dot{\boldsymbol{\varphi}} \cdot \boldsymbol{P} \cdot \widehat{\boldsymbol{N}} \mathrm{d} A \\
& +\int_{\partial \mathcal{V}_{0}^{+}} \dot{\boldsymbol{\varphi}} \cdot \boldsymbol{P} \cdot \widehat{\boldsymbol{N}} \mathrm{d} A+\int_{\mathcal{I}_{0}} \dot{\overline{\boldsymbol{\varphi}}} \cdot \overline{\boldsymbol{b}}_{0} \mathrm{~d} A+\int_{\partial \mathcal{I}_{0}} \dot{\overline{\boldsymbol{\varphi}}} \overline{\boldsymbol{P}} \cdot \widetilde{\boldsymbol{N}} \mathrm{d} L .
\end{aligned}
$$

Next, we impose invariance with respect to superposed rigid body motion to the working $\mathcal{W}_{0}$ as

$$
\mathcal{W}_{0}=\mathcal{W}_{0}(\dot{\boldsymbol{\varphi}}, \dot{\bar{\varphi}}) \stackrel{!}{=} \mathcal{W}_{0}(\dot{\boldsymbol{\varphi}}+\boldsymbol{v}+\boldsymbol{\omega} \times \boldsymbol{x}, \dot{\overline{\boldsymbol{\varphi}}}+\boldsymbol{v}+\boldsymbol{\omega} \times \overline{\boldsymbol{x}}) \quad \forall \boldsymbol{v}, \boldsymbol{\omega},
$$

in which $v$ and $\omega$ are constant (linear) velocity and constant angular velocity, respectively. Inserting Eq. (6) into Eq. (5) renders the global equation

$$
\begin{aligned}
& \int_{\mathcal{V}_{0}^{-}}[\boldsymbol{v}+\boldsymbol{\omega} \times \boldsymbol{x}] \cdot \boldsymbol{b}_{0} \mathrm{~d} V+\int_{\mathcal{V}_{0}^{+}}[\boldsymbol{v}+\boldsymbol{\omega} \times \boldsymbol{x}] \cdot \boldsymbol{b}_{0} \mathrm{~d} V+\int_{\partial \mathcal{V}_{0}^{-}}[\boldsymbol{v}+\boldsymbol{\omega} \times \boldsymbol{x}] \cdot \boldsymbol{P} \cdot \widehat{\boldsymbol{N}} \mathrm{d} A \\
& \quad+\int_{\partial \mathcal{V}_{0}^{+}}[\boldsymbol{v}+\boldsymbol{\omega} \times \boldsymbol{x}] \cdot \boldsymbol{P} \cdot \widehat{\boldsymbol{N}} \mathrm{d} A+\int_{\mathcal{I}_{0}}[\boldsymbol{v}+\boldsymbol{\omega} \times \overline{\boldsymbol{x}}] \cdot \overline{\boldsymbol{b}}_{0} \mathrm{~d} A+\int_{\partial \mathcal{I}_{0}}[\boldsymbol{v}+\boldsymbol{\omega} \times \overline{\boldsymbol{x}}] \cdot \overline{\boldsymbol{P}} \cdot \tilde{\boldsymbol{N}} \mathrm{d} L=0 \quad \forall \boldsymbol{v}, \boldsymbol{\omega} .
\end{aligned}
$$

Since Eq. (7) must hold for all arbitrary $\boldsymbol{v}$ and $\boldsymbol{\omega}$, we firstly set $\boldsymbol{\omega}=\mathbf{0}$ and derive the global balance of linear momentum as

$$
\begin{aligned}
& \int_{\mathcal{V}_{0}^{-}} \boldsymbol{v} \cdot \boldsymbol{b}_{0} \mathrm{~d} V+\int_{\mathcal{V}_{0}^{+}} \boldsymbol{v} \cdot \boldsymbol{b}_{0} \mathrm{~d} V+\int_{\partial \mathcal{V}_{0}^{-}} \boldsymbol{v} \cdot \boldsymbol{P} \cdot \widehat{\boldsymbol{N}} \mathrm{d} A \\
& \quad+\int_{\partial \mathcal{V}_{0}^{+}} \boldsymbol{v} \cdot \boldsymbol{P} \cdot \widehat{\boldsymbol{N}} \mathrm{d} A+\int_{\mathcal{I}_{0}} \boldsymbol{v} \cdot \overline{\boldsymbol{b}}_{0} \mathrm{~d} A+\int_{\partial \mathcal{I}_{0}} \boldsymbol{v} \cdot \overline{\boldsymbol{P}} \cdot \widetilde{\boldsymbol{N}} \mathrm{d} L=0 \quad \forall \boldsymbol{v} .
\end{aligned}
$$


Secondly, we derive the global balance of angular momentum by setting $\boldsymbol{v}=\mathbf{0}$ as

$$
\begin{aligned}
& \int_{\mathcal{V}_{0}^{-}} {[\boldsymbol{\omega} \times \boldsymbol{x}] \cdot \boldsymbol{b}_{0} \mathrm{~d} V+\int_{\mathcal{V}_{0}^{+}}[\boldsymbol{\omega} \times \boldsymbol{x}] \cdot \boldsymbol{b}_{0} \mathrm{~d} V+\int_{\partial \mathcal{V}_{0}^{-}}[\boldsymbol{\omega} \times \boldsymbol{x}] \cdot \boldsymbol{P} \cdot \widehat{\boldsymbol{N}} \mathrm{d} A } \\
& \quad+\int_{\partial \mathcal{V}_{0}^{+}}[\boldsymbol{\omega} \times \boldsymbol{x}] \cdot \boldsymbol{P} \cdot \widehat{\boldsymbol{N}} \mathrm{d} A+\int_{\mathcal{I}_{0}}[\boldsymbol{\omega} \times \overline{\boldsymbol{x}}] \cdot \overline{\boldsymbol{b}}_{0} \mathrm{~d} A+\int_{\partial \mathcal{I}_{0}}[\boldsymbol{\omega} \times \overline{\boldsymbol{x}}] \cdot \overline{\boldsymbol{P}} \cdot \tilde{\boldsymbol{N}} \mathrm{d} L=0 \quad \forall \boldsymbol{\omega} .
\end{aligned}
$$

Finally, through localization of Eqs. (8) and (9) to an infinitesimal subdomain in the bulk the classical balance of linear and angular momentum in the bulk are obtained as

$$
\operatorname{Div} \boldsymbol{P}+\boldsymbol{b}_{0}=\mathbf{0} \quad \text { and } \quad \boldsymbol{\varepsilon}:\left[\boldsymbol{F} \cdot \boldsymbol{P}^{\mathrm{t}}\right]=\mathbf{0} \Leftrightarrow \boldsymbol{P} \cdot \boldsymbol{F}^{\mathrm{t}}=\boldsymbol{F} \cdot \boldsymbol{P}^{\mathrm{t}},
$$

with $\boldsymbol{\varepsilon}$ being the permutation tensor. Detailed derivation of the localization procedure in the bulk is straightforward and is omitted here. In a similar fashion to the bulk, through localization of Eqs. (8) and (9) to an infinitesimal subdomain on the interface, the balance of linear and angular momentum on the interface are obtained as

$$
\overline{\operatorname{Div}} \overline{\boldsymbol{P}}+\overline{\boldsymbol{b}}_{0}+\llbracket \boldsymbol{t} \rrbracket=\mathbf{0} \quad \text { and } \quad \boldsymbol{\varepsilon}:\left[\llbracket \varphi \rrbracket \otimes\{[\boldsymbol{t}\}\}+\overline{\boldsymbol{F}} \cdot \overline{\boldsymbol{P}}^{\mathrm{t}}\right]=\mathbf{0} \quad \text { with } \quad \boldsymbol{t}=\boldsymbol{P} \cdot \overline{\boldsymbol{N}} .
$$

Detailed derivation of the localization procedure on the interface is given in Appendix B.1. It is of crucial importance to note that the local form of the balance of angular momentum on the interface is originally

$$
\varepsilon:\left[\llbracket \varphi \rrbracket \otimes\{[\boldsymbol{t}\}]+\overline{\boldsymbol{F}} \cdot \overline{\boldsymbol{P}}^{\mathrm{t}}+\{[\boldsymbol{\varphi}\}\} \otimes \llbracket t \rrbracket-\overline{\boldsymbol{\varphi}} \otimes \llbracket t \rrbracket\right]=0,
$$

and by constraining the interface motion to the mid-plane as $\bar{\varphi}=\{\{\varphi\}\}$ the last two terms cancel out together. Hence, Eq. (11) is valid based on this assumption and consequently, the interface motion is no longer arbitrary. For further details on the importance of the balance of angular momentum on the interface, see [107,108].

Constitutive laws. So far, we employed the invariance of working under a superposed rigid body motion to derive the local balance equations. Next, we start from working again and this time we view the working (4) as the external power that enters the thermodynamic framework. The main goal of this part is to derive thermodynamically consistent constitutive laws for the bulk and the interface via a Coleman-Noll-like procedure. The working density in the bulk and on the interface, respectively, reads

$$
\mathscr{W}_{0}=\boldsymbol{P}: \dot{\boldsymbol{F}} \quad \text { and } \quad \overline{\mathscr{W}}_{0}=\overline{\boldsymbol{P}}: \dot{\overline{\boldsymbol{F}}}+\{\{\boldsymbol{t}\}] \cdot \llbracket \dot{\boldsymbol{\varphi}} \rrbracket \quad \text { with } \quad \boldsymbol{t}=\boldsymbol{P} \cdot \overline{\boldsymbol{N}} .
$$

Detailed derivation and localization procedure to re-formulate the (global) working $\mathcal{W}_{0}$ of Eq. (4) in terms of the working densities $\mathscr{W}_{0}$ and $\overline{\mathscr{W}}_{0}$ in Eq. (12) is given in Appendix B.2. Note, the full form of the working density on the interface is

$$
\left.\overline{\mathscr{W}}_{0}=\overline{\boldsymbol{P}}: \dot{\overline{\boldsymbol{F}}}+\{\{\boldsymbol{t}\}] \cdot \llbracket \dot{\boldsymbol{\varphi}} \rrbracket+\llbracket t \rrbracket \cdot[\{\dot{\boldsymbol{\varphi}}\}\}-\dot{\overline{\boldsymbol{\varphi}}}\right],
$$

and due to constraining the interface motion to the mid-plane as $\bar{\varphi}=\{\{\varphi\}\}$ the last two terms cancel out. The interface working density $\overline{\mathscr{W}}_{0}$ in Eq. (12) is therefore valid based on the assumption that the interface is no longer arbitrary. Henceforth, we set this assumption on the interface motion and do not repeat the discussion again.

Next, we introduce the free energy as a Legendre transformation of the internal energy. Both bulk and the interface are endowed with their own free energy densities $\psi_{0}$ and $\bar{\psi}_{0}$, respectively. The bulk free energy density $\psi_{0}$ is defined per unit reference volume and similarly, the interface free energy density $\bar{\psi}_{0}$ is defined per unit reference area and both in the material configuration. From the definitions of the free energies, the Clausius-Duhem dissipation inequalities for the bulk and on the interface in the material configuration are

$$
\mathscr{D}_{0}=\mathscr{W}_{0}-\dot{\psi}_{0} \geq 0 \quad \text { and } \quad \overline{\mathscr{D}}_{0}=\overline{\mathscr{W}}_{0}-\dot{\bar{\psi}}_{0} \geq 0 .
$$

To further exploit the dissipation inequalities (13), we limit ourselves to (reversible) hyperelastic models in the bulk and on the interface. Inspired by the working densities (12), the bulk free energy density is assumed to be a function of the deformation gradient $\boldsymbol{F}$. Similarly, we allow the interface free energy to depend on the interface deformation gradient $\overline{\boldsymbol{F}}$, and the displacement jump across the interface $\llbracket \varphi \rrbracket$. The free energy densities in the bulk and on the interface thus read

$$
\psi_{0}=\psi_{0}(\boldsymbol{F}) \quad \text { and } \quad \bar{\psi}_{0}=\bar{\psi}_{0}(\overline{\boldsymbol{F}}, \llbracket \boldsymbol{\varphi} \rrbracket) .
$$


Inserting working densities (12) and free energy densities (14) into the dissipation inequalities (13) yields

$$
\begin{aligned}
& \mathscr{D}_{0}=\boldsymbol{P}: \dot{\boldsymbol{F}}-\frac{\partial \psi_{0}}{\partial \boldsymbol{F}}: \dot{\boldsymbol{F}} \geq 0, \\
& \overline{\mathscr{D}}_{0}=\overline{\boldsymbol{P}}: \dot{\overline{\boldsymbol{F}}}+\{\{\boldsymbol{t}\}] \cdot \llbracket \dot{\boldsymbol{\varphi}} \rrbracket-\frac{\partial \bar{\psi}_{0}}{\partial \overline{\boldsymbol{F}}}: \dot{\overline{\boldsymbol{F}}}-\frac{\partial \bar{\psi}_{0}}{\partial \llbracket \boldsymbol{\varphi} \rrbracket} \cdot \llbracket \dot{\boldsymbol{\varphi}} \rrbracket \geq 0 .
\end{aligned}
$$

Reordering the terms to better see the structure of the dissipation inequalities furnishes

$$
\begin{aligned}
& \mathscr{D}_{0}=\left[\boldsymbol{P}-\frac{\partial \psi_{0}}{\partial \boldsymbol{F}}\right]: \dot{\boldsymbol{F}} \geq 0, \\
& \overline{\mathscr{D}}_{0}=\left[\overline{\boldsymbol{P}}-\frac{\partial \bar{\psi}_{0}}{\partial \overline{\boldsymbol{F}}}\right]: \dot{\overline{\boldsymbol{F}}}+\left[\{\{\boldsymbol{t}\}\}-\frac{\partial \bar{\psi}_{0}}{\partial \llbracket \boldsymbol{\varphi} \rrbracket}\right] \cdot \llbracket \dot{\boldsymbol{\varphi}} \rrbracket \geq 0 .
\end{aligned}
$$

In order to extract the thermodynamically consistent constitutive laws within the context of equilibrium thermodynamics, it is customary to sufficiently satisfy the dissipation inequalities (16) by

$$
\left.\boldsymbol{P}=\frac{\partial \psi_{0}}{\partial \boldsymbol{F}} \quad \text { and } \quad \overline{\boldsymbol{P}}=\frac{\partial \bar{\psi}_{0}}{\partial \overline{\boldsymbol{F}}}, \quad\{\boldsymbol{t}\}\right\}=\frac{\partial \bar{\psi}_{0}}{\partial \llbracket \boldsymbol{\varphi} \rrbracket},
$$

which are indeed the constitutive laws for $\boldsymbol{P}, \overline{\boldsymbol{P}}$ and $\left\{\{\boldsymbol{t}\}\right.$, respectively. ${ }^{4}$

Remark. It is possible to introduce a hyperelastic model with damage for the bulk similar to [113-115] by choosing $\psi_{0}=\psi_{0}(\boldsymbol{F}, D)$ with $D$ being the damage parameter associated with the degradation of the bulk material. Introducing other internal variables to capture more complex material behavior introduces more notations and complexity without providing considerable insight into this contribution. For instance, we can allow the interface free energy to be a function of the interface deformation gradient $\overline{\boldsymbol{F}}$, the displacement jump across the interface $\llbracket \varphi \rrbracket$ as well as the interface damage parameter $\bar{D}$. Similar to the bulk, the interface damage parameter is essentially an evolving internal variable and can be interpreted as a general damage parameter on the interface and it may solely correspond to the degradation along the interface or across the interface or both. The free energy density of the interface accounting for the interface damage reads

$$
\bar{\psi}_{0}=\bar{\psi}_{0}(\overline{\boldsymbol{F}}, \llbracket \varphi \rrbracket, \bar{D}),
$$

and enters the interface dissipation inequality (14) as

$$
\left.\overline{\mathscr{D}}_{0}=\left[\overline{\boldsymbol{P}}-\frac{\partial \bar{\psi}_{0}}{\partial \overline{\boldsymbol{F}}}\right]: \dot{\boldsymbol{F}}+[\{\boldsymbol{t}\}\}-\frac{\partial \bar{\psi}_{0}}{\partial \llbracket \boldsymbol{\varphi} \rrbracket}\right] \cdot \llbracket \dot{\boldsymbol{\varphi}} \rrbracket-\frac{\partial \bar{\psi}_{0}}{\partial \bar{D}} \dot{\bar{D}} \geq 0,
$$

which results in the thermodynamically consistent constitutive and evolutions laws on the interface as

$$
\overline{\boldsymbol{P}}=\frac{\partial \bar{\psi}_{0}}{\partial \overline{\boldsymbol{F}}}, \quad\{\{\boldsymbol{t}\}\}=\frac{\partial \bar{\psi}_{0}}{\partial \llbracket \boldsymbol{\varphi} \rrbracket}, \quad \frac{\partial \bar{\psi}_{0}}{\partial \bar{D}} \dot{\bar{D}} \leq 0 .
$$

\footnotetext{
${ }^{4}$ Clearly, the interface normal $\bar{n}$ depends on the interface deformation gradient $\overline{\boldsymbol{F}}$. However, if we allow the interface free energy to depend explicitly on $\overline{\boldsymbol{n}}$, using the relation $\partial \overline{\boldsymbol{n}} / \partial \overline{\boldsymbol{F}}=-\overline{\boldsymbol{n}} \bar{\otimes} \overline{\boldsymbol{F}}^{-\mathrm{t}}$ proven in Appendix B.3, we expand the interface stress

$$
\begin{aligned}
& \overline{\boldsymbol{P}}=\left.\frac{\partial \bar{\psi}_{0}}{\partial \overline{\boldsymbol{F}}}\right|_{\overline{\boldsymbol{n}}}+\frac{\partial \bar{\psi}_{0}}{\partial \overline{\boldsymbol{n}}} \cdot \frac{\partial \overline{\boldsymbol{n}}}{\partial \overline{\boldsymbol{F}}}=\left.\frac{\partial \bar{\psi}_{0}}{\partial \overline{\boldsymbol{F}}}\right|_{\overline{\boldsymbol{n}}}-\frac{\partial \bar{\psi}_{0}}{\partial \overline{\boldsymbol{n}}} \cdot\left[\overline{\boldsymbol{n}} \bar{\otimes}^{-\mathrm{t}}\right]=\left.\frac{\partial \bar{\psi}_{0}}{\partial \overline{\boldsymbol{F}}}\right|_{\overline{\boldsymbol{n}}}-\overline{\boldsymbol{n}} \otimes\left[\frac{\partial \bar{\psi}_{0}}{\partial \overline{\boldsymbol{n}}} \cdot \overline{\boldsymbol{F}}^{-\mathrm{t}}\right]=\left.\frac{\partial \bar{\psi}_{0}}{\partial \overline{\boldsymbol{F}}}\right|_{\overline{\boldsymbol{n}}}-\overline{\boldsymbol{n}} \otimes \overline{\boldsymbol{S}} \\
& \quad \text { with } \overline{\boldsymbol{S}}=\frac{\partial \bar{\psi}_{0}}{\partial \overline{\boldsymbol{n}}} \cdot \overline{\boldsymbol{F}}^{-\mathrm{t}}=\frac{\partial \bar{\psi}_{t}}{\partial \overline{\boldsymbol{n}}} \cdot \overline{\operatorname{Cof}} \overline{\boldsymbol{F}}
\end{aligned}
$$

in which $\bar{S}$ is a vector tangent to the interface on the material configuration and $\bar{\psi}_{t}$ is the interface free energy density in the spatial configuration. The particular and unfamiliar format of the term $\overline{\boldsymbol{n}} \otimes \overline{\boldsymbol{S}}$ has attracted special attention in the literature [109-111,105,112] and ties to the surface shear concept.
} 
Table 1

Summary of governing equations and constitutive laws together with some definitions and notations in the bulk and on the interface. Note, the governing equations rely on the assumption that the interface motion is exactly in the middle of the finite thickness interphase or $\bar{\varphi}=\{\varphi\}$. Furthermore, $\boldsymbol{t}$ denotes the interface traction defined as $\boldsymbol{t}=\boldsymbol{P} \cdot \overline{\boldsymbol{N}}$. The interface unit normal $\overline{\boldsymbol{N}}$ points from the minus to the plus side of the interface and can be computed as $\overline{\boldsymbol{N}}= \pm \overline{\boldsymbol{G}}_{1} \times \overline{\boldsymbol{G}}_{2} /\left|\overline{\boldsymbol{G}}_{1} \times \overline{\boldsymbol{G}}_{2}\right|$ whereby the \pm sign indicates that this formulation cannot determine the direction of the normal and that shall be constrained with the surrounding bulk. All relations are associated with the material configuration.

\begin{tabular}{|c|c|c|}
\hline & In bulk $\mathcal{V}_{0}$ & On interface $\mathcal{I}_{0}$ \\
\hline $\begin{array}{l}\text { bal. lin. mom. } \\
\text { bal. ang. mom. } \\
\text { const. evol. law }\end{array}$ & $\begin{array}{l}\operatorname{Div} \boldsymbol{P}+\boldsymbol{b}_{0}=\mathbf{0} \text { subject to } \widehat{\boldsymbol{b}}=\boldsymbol{P} \cdot \widehat{\boldsymbol{N}} \text { on } \partial \mathcal{V}_{0} \\
\boldsymbol{\varepsilon}:\left[\boldsymbol{F} \cdot \boldsymbol{P}^{\mathrm{t}}\right]=\mathbf{0} \\
\boldsymbol{P}=\partial \psi_{0} / \partial \boldsymbol{F}\end{array}$ & $\begin{array}{l}\overline{\operatorname{Div}} \overline{\boldsymbol{P}}+\overline{\boldsymbol{b}}_{0}+\llbracket \boldsymbol{t} \rrbracket=\mathbf{0} \text { subject to } \widetilde{\boldsymbol{b}}=\overline{\boldsymbol{P}} \cdot \widetilde{\boldsymbol{N}} \text { on } \partial \mathcal{I}_{0} \\
\boldsymbol{\varepsilon}:\left[\overline{\boldsymbol{F}} \cdot \overline{\boldsymbol{P}}^{\mathrm{t}}+\llbracket \boldsymbol{\varphi} \rrbracket \otimes\{\boldsymbol{t}\}\right]=\mathbf{0} \\
\overline{\boldsymbol{P}}=\partial \bar{\psi}_{0} / \partial \overline{\boldsymbol{F}},\{\boldsymbol{t} \boldsymbol{t}\}=\partial \bar{\psi}_{0} / \partial \llbracket \boldsymbol{\varphi} \rrbracket\end{array}$ \\
\hline non-lin. map & $\varphi \quad$ or $\quad \varphi=\varphi(X)$ & $\bar{\varphi} \quad$ or $\quad \bar{\varphi}=\bar{\varphi}(\bar{X})$ \\
\hline lin. tan. map & $\boldsymbol{F} \quad$ or $\quad \mathrm{d} \boldsymbol{\varphi}=\boldsymbol{F} \cdot \mathrm{d} \boldsymbol{X}$ & $\overline{\boldsymbol{F}} \quad$ or $\quad \mathrm{d} \overline{\boldsymbol{\varphi}}=\overline{\boldsymbol{F}} \cdot \mathrm{d} \overline{\boldsymbol{X}}$ \\
\hline lin. nor. map & $\operatorname{Cof} \boldsymbol{F} \quad$ or $\quad \mathrm{d} \boldsymbol{a}=\operatorname{Cof} \boldsymbol{F} \cdot \mathrm{d} \boldsymbol{A}$ & $\overline{\operatorname{Cof}} \overline{\boldsymbol{F}} \quad$ or $\quad \mathrm{d} \overline{\boldsymbol{l}}=\overline{\operatorname{Cof}} \overline{\boldsymbol{F}} \cdot \mathrm{d} \overline{\boldsymbol{L}}$ \\
\hline co-var. bas. & $G_{1}, G_{2}, G_{3}$ & $\overline{\boldsymbol{G}}_{1}, \overline{\boldsymbol{G}}_{2}$ \\
\hline contra-var. bas. & $G^{1}, G^{2}, G^{3}$ & $\overline{\boldsymbol{G}}^{1}, \overline{\boldsymbol{G}}^{2}$ \\
\hline Identity & $I=G_{1} \otimes G^{1}+G_{2} \otimes G^{2}+G_{3} \otimes G^{3}$ & $\overline{\boldsymbol{I}}=\overline{\boldsymbol{G}}_{1} \otimes \overline{\boldsymbol{G}}^{1}+\overline{\boldsymbol{G}}_{2} \otimes \overline{\boldsymbol{G}}^{2}=\boldsymbol{I}-\overline{\boldsymbol{N}} \otimes \overline{\boldsymbol{N}}$ \\
\hline Gradient & $\operatorname{Grad}\{\bullet\}=\partial\{\bullet\} / \Theta^{i} \otimes \boldsymbol{G}^{i}, \quad 1 \leq i \leq 3$ & $\overline{\operatorname{Grad}}\{\overline{\boldsymbol{\theta}}\}=\partial\{\overline{\boldsymbol{\theta}}\} / \Theta^{\alpha} \otimes \overline{\boldsymbol{G}}^{\alpha}, \quad 1 \leq \alpha \leq 2$ \\
\hline Divergence & $\operatorname{Div}\{\bullet\}=\partial\{\bullet\} / \Theta^{i} \cdot \boldsymbol{G}^{i}, \quad 1 \leq i \leq 3$ & $\overline{\operatorname{Div}}\{\bar{\bullet}\}=\partial\{\bar{\bullet}\} / \Theta^{\alpha} \cdot \overline{\boldsymbol{G}}^{\alpha}, \quad 1 \leq \alpha \leq 2$ \\
\hline Determinant & $\operatorname{Det}\{\bullet\}=\frac{\{\bullet\} \cdot \boldsymbol{G}_{1} \cdot\left[\{\bullet\} \cdot \boldsymbol{G}_{2} \times\{\bullet\} \cdot \boldsymbol{G}_{3}\right]}{\boldsymbol{G}_{1} \cdot\left[\boldsymbol{G}_{2} \times \boldsymbol{G}_{3}\right]}$ & $\overline{\operatorname{Det}}\{\bar{\bullet}\}=\frac{\left|\{\overline{\boldsymbol{\theta}}\} \cdot \overline{\boldsymbol{G}}_{1} \times\{\overline{\boldsymbol{\theta}}\} \cdot \overline{\boldsymbol{G}}_{2}\right|}{\left|\overline{\boldsymbol{G}}_{1} \times \overline{\boldsymbol{G}}_{2}\right|}$ \\
\hline Cofactor & $\operatorname{Cof}\{\bullet\}=\operatorname{Det}\{\bullet\}\{\bullet\}^{-t}$ & $\overline{\operatorname{Cof}}\{\bar{\bullet}\}=\overline{\operatorname{Det}}\{\bar{\bullet}\}\{\bar{\bullet}\}^{-t}$ \\
\hline
\end{tabular}

So far, the fundamental concepts of general imperfect interfaces have been elaborated. Note, the general imperfect interface model at hand reduces to the cohesive zone model if $\bar{\psi}_{0}=\bar{\psi}_{0}(\llbracket \varphi \rrbracket)$. Also, it recovers the interface elasticity model if $\bar{\psi}_{0}=\bar{\psi}_{0}(\overline{\boldsymbol{F}})$. Equipped with the governing equations and constitutive laws at hand, we proceed in Section 2.2 with the computational homogenization of micro-structures including general imperfect interfaces. Table 1 gathers the governing equations and constitutive laws together with some definitions and notations in the bulk and on the interface.

\subsection{Computational homogenization}

The purpose of this section is to study a heterogeneous material composed of an inclusion within the matrix. The interface between the constituents is endowed with its own energetic structure. We allow the matrix to include only one inclusion for simplicity but considering several inclusions does not change the framework. Throughout this work, similar to the classical first-order computational homogenization, we assume that the common assumption of length-scale separation associated with homogenization holds. Therefore, the macroscopic problem is large enough compared to the length scale associated with the microscale problem. However, in contrast to the classical firstorder computational homogenization, the microscale possesses a physical length-scale. In analogy to the derivation of equations governing the interface in Section 2.1, the micro-to-macro transition is formulated only in the Lagrangian setting. However, describing the problem in the Eulerian setting does not affect the primary objective of this manuscript, namely, to account for interfaces within the computational homogenization framework. For further details on computational homogenization and its underlying assumptions refer to the extensive references listed in the introduction.

\subsubsection{Macroscale}

Consider a continuum body that takes the material configuration ${ }^{\mathrm{M}_{\mathcal{B}}}$ at time $t=0$ and the spatial configuration $\mathrm{M}_{\mathcal{B}_{t}}$ at any time $t$ as shown in Fig. 3. At the macro level the outward surface unit normal in the material and spatial configurations is denoted ${ }^{\mathrm{M}} \widehat{\boldsymbol{N}}$ and ${ }^{\mathrm{M}} \widehat{\boldsymbol{n}}$, respectively. A material point in the macroscale is characterized by the position vector ${ }^{{ }^{M}} \boldsymbol{X}$ and is mapped to its spatial counterpart ${ }^{\mathrm{M}} \boldsymbol{x}$ via the non-linear deformation map ${ }^{\mathrm{M}} \boldsymbol{\varphi}$ as ${ }^{\mathrm{M}} \boldsymbol{x}={ }^{\mathrm{M}} \boldsymbol{\varphi}\left({ }^{\mathrm{M}} \boldsymbol{X}\right)$. The corresponding macro deformation gradient ${ }^{\mathrm{M}} \boldsymbol{F}$ maps (linearly) the line element $\mathrm{d}^{\mathrm{M}} \boldsymbol{X}$ in the material configuration to 


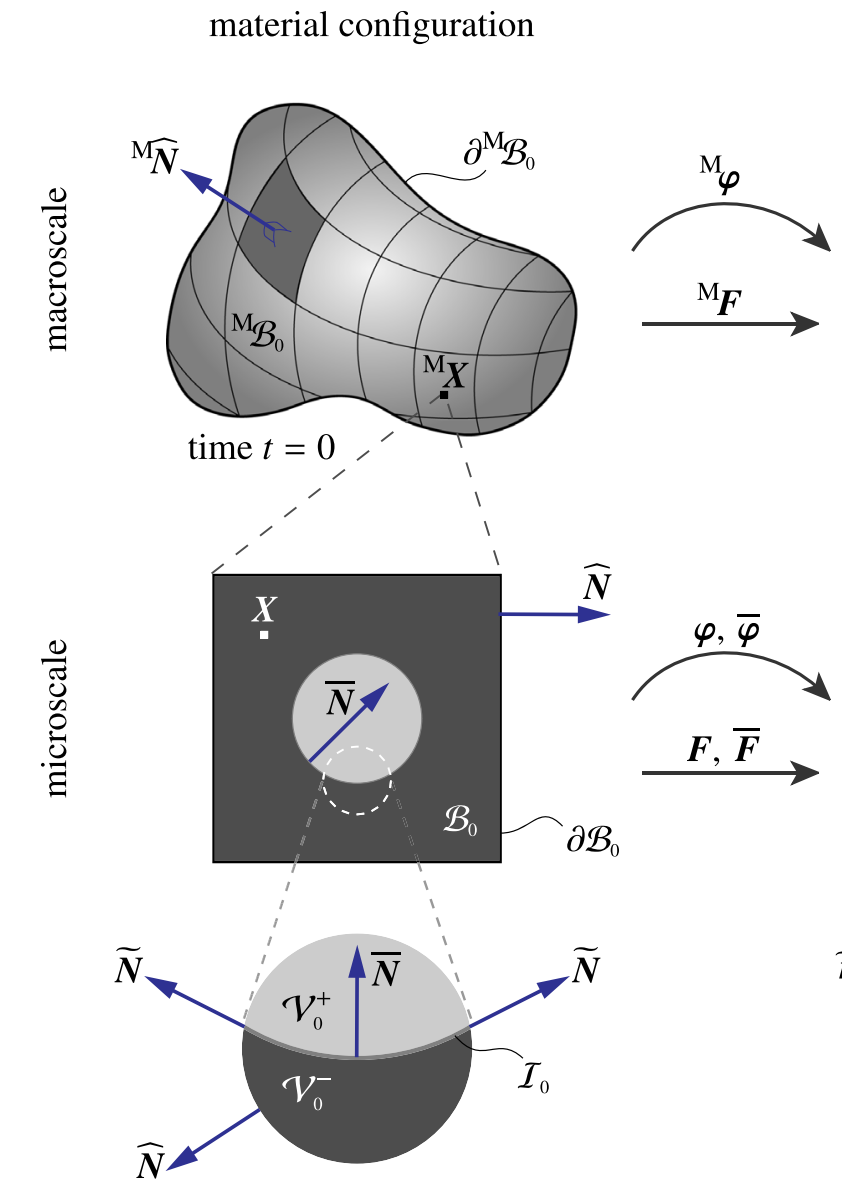

spatial configuration

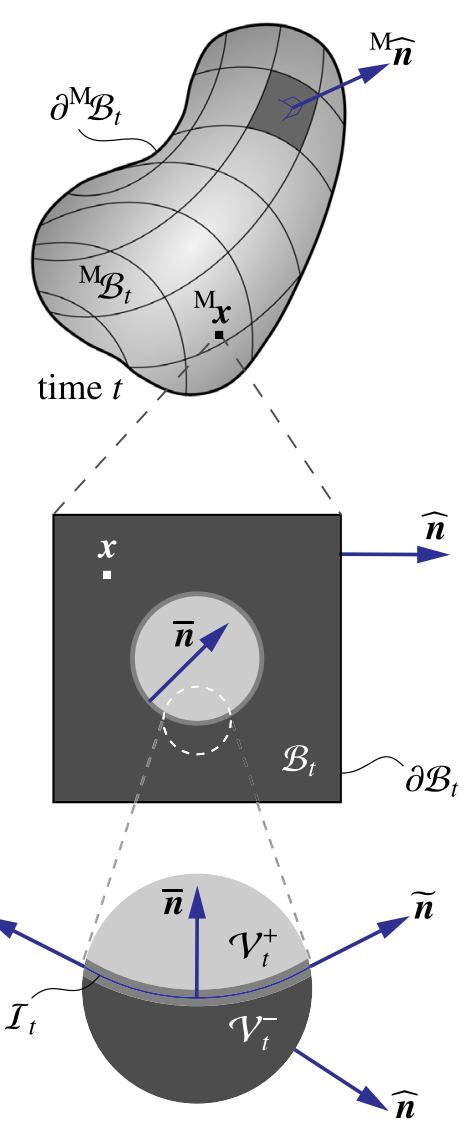

Fig. 3. A graphical summary of micro-to-macro transition of continua that accounts for interfaces at the microscale. The non-linear deformation map $\mathrm{M}_{\varphi}$ maps the macroscopic material configuration $\mathrm{M}_{\mathcal{B}_{0}}$ to the spatial configuration $\mathrm{M}_{\mathcal{B}_{t}}$. The material configuration at the microscale $\mathcal{B}_{0}$ represents a RVE and is mapped to its spatial counterpart via the motion $\varphi$. Constitutive laws at the microscale are assumed to be known and the goal is to compute the macroscopic response through homogenizing the response of the underlying micro-structure. In particular, at the microscale the interface between different constituents is considered and plays a crucial role on the overall behavior of the material.

the spatial line element $\mathrm{d}^{\mathrm{M}} \boldsymbol{x}$ according to

$$
\mathrm{d}^{\mathrm{M}} \boldsymbol{x}=\mathrm{d}^{\mathrm{M}} \boldsymbol{F} \cdot \mathrm{d}^{\mathrm{M}} \boldsymbol{X} \quad \text { and } \quad \mathrm{M}_{\boldsymbol{F}}={ }^{\mathrm{M}} \operatorname{Grad}^{\mathrm{M}} \boldsymbol{\varphi} \quad \text { with } \quad{ }^{\mathrm{M}} \mathrm{Grad}\{\bullet\}=\partial\{\bullet\} / \partial^{\mathrm{M}} \boldsymbol{X} .
$$

The governing equations at the macroscale are the balances of linear momentum and angular momentum. In the absence of inertial effects, the balance of linear momentum reads

$$
{ }^{\mathrm{M}}{ }_{\operatorname{Div}}{ }^{\mathrm{M}} \boldsymbol{P}+{ }^{\mathrm{M}} \boldsymbol{b}_{0}^{\mathrm{p}}=\mathbf{0} \quad \text { in }{ }^{\mathrm{M}_{\mathcal{B}}} \quad \text { subject to } \quad{ }^{\mathrm{M}} \boldsymbol{P} \cdot{ }^{\mathrm{M}} \widehat{\boldsymbol{N}}-{ }^{\mathrm{M}} \widehat{\boldsymbol{b}}_{0}^{\mathrm{p}}=\mathbf{0} \quad \text { on } \partial^{\mathrm{M}} \mathcal{B}_{0}^{\mathrm{N}},
$$

with ${ }^{M} \boldsymbol{b}_{0}^{\mathrm{p}}$ being the macroscopic body force density in the material configuration and ${ }^{\mathrm{M}} \boldsymbol{P}$ the macro Piola stress. The prescribed traction per unit reference area in the material configuration is denoted ${ }^{\mathrm{M}} \widehat{\boldsymbol{b}}_{0}^{\mathrm{p}}$ and is applied on the Neumann boundary $\partial^{\mathrm{M}} \mathcal{B}_{0}^{\mathrm{N}}$. The local form of balance of angular momentum in the material configuration is

$$
{ }^{\mathrm{M}} \boldsymbol{P} \cdot{ }^{\mathrm{M}} \boldsymbol{F}^{\mathrm{t}}={ }^{\mathrm{M}} \boldsymbol{F} \cdot{ }^{\mathrm{M}} \boldsymbol{P}^{\mathrm{t}} .
$$

\subsubsection{Microscale}

The material configuration at the microscale is denoted $\mathcal{B}_{0}$ and is assumed to be statistically representative of the material. The configuration $\mathcal{B}_{0}$ defines the representative volume element (RVE) in the material configuration and its external boundary is denoted $\partial \mathcal{B}_{0}$ with outward unit normal $\widehat{N}$, see Fig. 3 . The spatial configuration at the microscale 
is defined analogously. The interface $\mathcal{I}_{0}$ in the material configuration is the boundary of the inclusion or equivalently the internal boundary of the matrix. The domains $\mathcal{B}_{0}^{-}$and $\mathcal{B}_{0}^{+}$represent the matrix and inclusion, respectively with their boundaries as

$$
\partial \mathcal{B}_{0}^{-}=\partial \mathcal{B}_{0} \cup \mathcal{I}_{0}, \quad \partial \mathcal{B}_{0}^{+}=\mathcal{I}_{0}, \quad \mathcal{I}_{0}^{-}=\partial \mathcal{B}_{0}^{-} \cap \mathcal{I}_{0}, \quad \mathcal{I}_{0}^{+}=\partial \mathcal{B}_{0}^{+} \cap \mathcal{I}_{0} .
$$

The plus and minus sides of the interface coincide geometrically in the material configuration, nevertheless, we need to distinguish between them since they no longer coincide in the spatial configuration. For simplicity the interface is assumed to be smooth and does not possess a sharp kink or cusp. The unit normal to $\mathcal{I}_{0}$ pointing from the minus to the plus side of the interface is denoted $\bar{N}$. The arbitrary cutout volume around the interface in the material configuration is denoted $\mathcal{V}_{0}$ and is essentially identical to the $\mathcal{V}_{0}$ of Section 2.1. In this manuscript, we assume that the inclusion, and consequently the interface, is entirely enclosed within the RVE and the external boundary of the RVE is chosen such that it has no intersection with the interface $\mathcal{I}_{0}$ and therefore $\partial \mathcal{I}_{0}=\emptyset$. This assumption only simplifies the derivations and does not influence the fundamental concepts.

Classical continuum mechanics assigns the free energy only to the bulk material. The same holds for available homogenization schemes. Interface elasticity theory, though, requires additionally an independent interface energy assigned to the interfaces $\mathcal{I}_{0}$. At the micro level, we employ the interface enhanced continuum theory elaborated in Section 2.1 while having the classical continuum theory at the macro level. This is motivated by the fact that due to the small dimensions and, consequently, large area to volume ratio of the micro problem, the interface effects are not negligible at the microscale. In general, independent free energy densities shall be assigned to the interface and the bulk at the microscale.

In a near-identical fashion to the macroscopic problem, the kinematics of the micro problem are presented now. Let $\boldsymbol{X}$ be the position vector of a point in $\mathcal{B}_{0}$ that is mapped via the non-linear deformation map to its counterpart $\varphi$ in the spatial configuration $\mathcal{B}_{t}$. The points on the material interface $\bar{X}:=\left.X\right|_{\mathcal{I}_{0}}$ are mapped to $\overline{\boldsymbol{x}}$ via $\bar{\varphi}$ and $\bar{\varphi}=\left\{\{\varphi\}||_{\mathcal{I}_{0}}\right.$. The material line elements $\mathrm{d} \boldsymbol{X} \in \mathcal{B}_{0}$ are mapped to $\mathrm{d} \boldsymbol{x} \in \mathcal{B}_{t}$ via the linear deformation map $\boldsymbol{F}=\operatorname{Grad} \boldsymbol{\varphi}$. On the interface $\mathcal{I}_{0}$ the material line elements $\mathrm{d} \overline{\boldsymbol{X}}$ are mapped to the spatial (interface) line elements $\mathrm{d} \overline{\boldsymbol{x}}$ via the interface deformation gradient $\overline{\boldsymbol{F}}$ as $\mathrm{d} \overline{\boldsymbol{x}}=\overline{\boldsymbol{F}} \cdot \mathrm{d} \overline{\boldsymbol{X}}$ with tensor $\overline{\boldsymbol{F}}=\overline{\mathrm{Grad}} \overline{\boldsymbol{\varphi}}=\boldsymbol{F} \cdot \overline{\boldsymbol{I}}$ where $\overline{\boldsymbol{I}}=\boldsymbol{I}-\overline{\boldsymbol{N}} \otimes \overline{\boldsymbol{N}}$ denotes the interface identity tensor or rather the interface projection. For the microscale problem of interest, the governing equations are those in Section 2.1 and are summarized in Table 1.

In passing, we mention that the body force density in the bulk $\boldsymbol{b}_{0}$ is neglected at the microscale. This assumption is customary within the context of the micro-to-macro transition and is based on the argument that $\boldsymbol{b}_{0}$ is a force density per volume. Therefore, due to the scale separation associated with homogenization, the force densities per volume are negligible compared to the force densities per area such as traction $\widehat{\boldsymbol{b}}_{0}$ and the interface force density $\overline{\boldsymbol{b}}_{0}$. One can argue though, that the interface force density $\overline{\boldsymbol{b}}_{0}$ shall vanish in a mechanical problem as there is no clear mechanism to prescribe it. Henceforth, we skip the interface force density $\overline{\boldsymbol{b}}_{0}$ for the sake of simplicity. ${ }^{5}$

\subsubsection{Micro-to-macro transition}

Macroscopic quantities are related to their micro counterparts through volume averaging over the RVE and fundamental reasoning detailed in this section. It proves convenient to define the following averaging operator in the material configuration $\langle\{\bullet\}\rangle_{\{0\}}$ as the integral of $\{\bullet\}$ over the domain $\{0\}$ divided by the volume $\mathscr{V}_{0}$ as

$$
\langle\{\bullet\}\rangle_{\{0\}}=\frac{1}{\mathscr{V}_{0}} \int_{\{0\}}\{\bullet\} \mathrm{d}\{0\},
$$

such as

$$
\langle\{\bullet\}\rangle_{\mathcal{B}_{0}^{-}}=\frac{1}{\mathscr{V}_{0}} \int_{\mathcal{B}_{0}^{-}}\{\bullet\} \mathrm{d} V, \quad\langle\{\bullet\}\rangle_{\mathcal{B}_{0}^{+}}=\frac{1}{\mathscr{V}_{0}} \int_{\mathcal{B}_{0}^{+}}\{\bullet\} \mathrm{d} V, \quad\langle\{\bar{\bullet}\}\rangle_{\mathcal{I}_{0}}=\frac{1}{\mathscr{V}_{0}} \int_{\mathcal{I}_{0}}\{\bar{\bullet}\} \mathrm{d} A,
$$

\footnotetext{
5 Although we neglect the interface force density $\overline{\boldsymbol{b}}_{0}$ for a purely mechanical case, it is crucial to account for it in multi-physics problems. For instance, imagine coated particles in a matrix where the coating is sensitive to external, e.g. magnetic or electric, fields that induce an interface force density $\overline{\boldsymbol{b}}_{0}$ under the influence of an externally applied field. Within the context of homogenization, it is rather straightforward to introduce $\bar{b}_{0}$ in the framework and formally follow the same steps.
} 
and in particular, for a smoothly defined field $\{\bullet\}$ in $\mathcal{B}_{0}^{ \pm}$, the (classical) average operator $\langle\{\bullet\}\rangle$ relates to our notation as

$$
\langle\{\bullet\}\rangle_{\mathcal{B}_{0}}=\frac{1}{\mathscr{V}_{0}} \int_{\mathcal{B}_{0}^{-}}\{\bullet\} \mathrm{d} V+\frac{1}{\mathscr{V}_{0}} \int_{\mathcal{B}_{0}^{+}}\{\bullet\} \mathrm{d} V \quad \Rightarrow \quad\langle\{\bullet\}\rangle=\langle\{\bullet\}\rangle_{\mathcal{B}_{0}} .
$$

The volume $\mathscr{V}_{0}$ is the total volume surrounded by the (external) boundary $\partial \mathcal{B}_{0}$ and can be understood as the total volume of the inclusion together with the matrix. The identity

$$
\int_{\partial \mathcal{B}_{0}} \widehat{\boldsymbol{N}} \otimes \boldsymbol{X} \mathrm{d} A=\mathscr{V}_{0} \boldsymbol{I} \quad \Rightarrow \quad\langle\widehat{\boldsymbol{N}} \otimes \boldsymbol{X}\rangle_{\partial \mathcal{B}_{0}}=\boldsymbol{I},
$$

is frequently used and follows directly from the gradient theorem as

$$
\int_{\partial \mathcal{B}_{0}} \widehat{\boldsymbol{N}} \otimes \boldsymbol{X} \mathrm{d} A=\int_{\mathcal{B}_{0}}[\operatorname{Grad} \boldsymbol{X}]^{\mathrm{t}} \mathrm{d} V=\int_{\mathcal{B}_{0}} \boldsymbol{I} \mathrm{d} V=\mathscr{V}_{0} \boldsymbol{I} .
$$

Average deformation gradient theorem. In order to relate the micro deformation gradient to the macro deformation gradient, motivated by classical homogenization, we develop an average deformation gradient theorem. ${ }^{6}$ The classical average deformation gradient theorem is extended to account for interface contributions. Our derivations yield that the extended deformation gradient theorem includes extra terms only due to the deformation jump across the interface but not the deformation gradient along the interface. Therefore, the "extended" format of the average deformation gradient theorem formally reduces to that of the classical homogenization with deformation jumps presented earlier in [60].

Theorem. Let $\boldsymbol{F}_{c}$ be a given constant deformation gradient tensor and $\partial \mathcal{B}_{0}$ be the external boundary of the domain $\mathcal{B}_{0}$ with outward unit normal $\widehat{\boldsymbol{N}}$ as illustrated in Fig. 3. If $\boldsymbol{\varphi}^{p}=\boldsymbol{F}_{c} \cdot \boldsymbol{X}$ is prescribed on the entire $\partial \mathcal{B}_{0}$, then we have $\langle\boldsymbol{F}\rangle_{\mathcal{B}_{0}}+\langle\llbracket \varphi \rrbracket \otimes \overline{\boldsymbol{N}}\rangle_{\mathcal{I}_{0}}=\boldsymbol{F}_{c}$ or alternatively, $\langle\boldsymbol{F}\rangle_{\mathcal{B}_{0}}+\langle\llbracket \boldsymbol{F} \rrbracket \cdot \overline{\boldsymbol{I}}\rangle_{\mathcal{I}_{0}}=\boldsymbol{F}_{c}$.

Proof. In order to prove the extended average deformation gradient theorem, we use the lemma

$$
\langle\boldsymbol{F}\rangle_{\mathcal{B}_{0}}+\langle\llbracket \varphi \rrbracket \otimes \bar{N}\rangle_{\mathcal{I}_{0}}=\langle\boldsymbol{\varphi} \otimes \widehat{N}\rangle_{\partial \mathcal{B}_{0}}
$$

proven in Appendix C.1. Replacing the motion $\boldsymbol{\varphi}$ with its prescribed value $\varphi^{\mathrm{p}}=\boldsymbol{F}_{\mathrm{c}} \cdot \boldsymbol{X}$ in the lemma (25) and using the identity (24), yields

$$
\langle\boldsymbol{F}\rangle_{\mathcal{B}_{0}}+\langle\llbracket \varphi \rrbracket \otimes \overline{\boldsymbol{N}}\rangle_{\mathcal{I}_{0}}=\left\langle\boldsymbol{\varphi}^{\mathrm{p}} \otimes \widehat{\boldsymbol{N}}\right\rangle_{\partial \mathcal{B}_{0}}=\left\langle\boldsymbol{F}_{\mathrm{c}} \cdot \boldsymbol{X} \otimes \widehat{\boldsymbol{N}}\right\rangle_{\partial \mathcal{B}_{0}}=\boldsymbol{F}_{\mathrm{c}} \cdot\langle\boldsymbol{X} \otimes \widehat{\boldsymbol{N}}\rangle_{\partial \mathcal{B}_{0}}=\boldsymbol{F}_{\mathrm{c}} \cdot \boldsymbol{I}=\boldsymbol{F}_{\mathrm{c}}
$$

The average deformation gradient theorem states that when a body is subject to the linear displacement boundary conditions defined above, with $\boldsymbol{F}_{\mathrm{c}}$ being a constant tensor, the integral of the bulk deformation gradient plus the integral of the jump of the deformation gradient projected onto the interface averaged over the entire body is the same as $\boldsymbol{F}_{\mathrm{c}}$ regardless of the complexity of the deformation within the RVE domain. In view of the micro-to-macro transition, the average deformation gradient theorem motivates the definition of the macro deformation gradient as

$$
{ }^{\mathrm{M}} \boldsymbol{F}:=\langle\boldsymbol{F}\rangle_{\mathcal{B}_{0}}+\langle\llbracket \varphi \rrbracket \otimes \overline{\boldsymbol{N}}\rangle_{\mathcal{I}_{0}},
$$

or alternatively

$$
\mathrm{M}_{\boldsymbol{F}}:=\langle\boldsymbol{\varphi} \otimes \widehat{\boldsymbol{N}}\rangle_{\partial \mathcal{B}_{0}} .
$$

Clearly, in the absence of the deformation jump across the interface, i.e. coherent interfaces, the relation (26) reduces to the classical average deformation gradient theorem. More interestingly, even in the presence of deformation jumps, the alternative definition of the macro deformation gradient as the boundary integral (27) is identical to its format in the classical homogenization.

Average stress theorem. In order to relate the micro stresses to the macroscopic ones, motivated by classical homogenization, we develop an average stress theorem. However, the classical average stress theorem needs to be

\footnotetext{
6 The average deformation gradient theorem is often referred to as average strain theorem due to its origin in linear elasticity. Nevertheless, within the finite deformation setting of this contribution we consistently refer to it as the average deformation gradient theorem.
} 
extended in order to account for interface stresses. In contrast to the extended average deformation gradient theorem, our derivations yield that the extended average stress theorem includes extra terms only due to the interface stresses along the interface but not traction jumps across the interface.

Theorem. Let $\boldsymbol{P}_{c}$ be a given constant stress tensor and $\partial \mathcal{B}_{0}$ the external boundary of the domain $\mathcal{B}_{0}$ with outward unit normal $\widehat{\boldsymbol{N}}$ as shown in Fig. 3. If $\boldsymbol{P} \cdot \widehat{\boldsymbol{N}}=\widehat{\boldsymbol{b}}_{0}^{\mathrm{p}}=\boldsymbol{P}_{c} \cdot \widehat{\boldsymbol{N}}$ is prescribed on the entire $2 \mathcal{B}_{0}$, then $\langle\boldsymbol{P}\rangle_{\mathcal{B}_{0}}+\langle\overline{\boldsymbol{P}}\rangle_{\mathcal{I}_{0}}=\boldsymbol{P}_{c}$.

Proof. In order to prove the extended average stress theorem, we use the lemma

$$
\langle\boldsymbol{P}\rangle_{\mathcal{B}_{0}}+\langle\overline{\boldsymbol{P}}\rangle_{\mathcal{I}_{0}}=\left\langle\widehat{\boldsymbol{b}}_{0} \otimes \boldsymbol{X}\right\rangle_{\partial \mathcal{B}_{0}}
$$

proven in Appendix C.2. Replacing the traction $\widehat{\boldsymbol{b}}_{0}$ with its prescribed value $\widehat{\boldsymbol{b}}_{0}^{\mathrm{p}}=\boldsymbol{P}_{\mathrm{c}} \cdot \widehat{\boldsymbol{N}}$ in the lemma (28) and using the identity (24), yields

$$
\langle\boldsymbol{P}\rangle_{\mathcal{B}_{0}}+\langle\overline{\boldsymbol{P}}\rangle_{\mathcal{I}_{0}}=\left\langle\widehat{\boldsymbol{b}}_{0}^{\mathrm{p}} \otimes \boldsymbol{X}\right\rangle_{\partial \mathcal{B}_{0}}=\left\langle\boldsymbol{P}_{\mathrm{c}} \cdot \widehat{\boldsymbol{N}} \otimes \boldsymbol{X}\right\rangle_{\partial \mathcal{B}_{0}}=\boldsymbol{P}_{\mathrm{c}} \cdot\langle\widehat{\boldsymbol{N}} \otimes \boldsymbol{X}\rangle_{\partial \mathcal{B}_{0}}=\boldsymbol{P}_{\mathrm{c}} \cdot \boldsymbol{I}=\boldsymbol{P}_{\mathrm{c}}
$$

The average stress theorem essentially states that when a body is subject to traction boundary conditions as defined above, the integral of the bulk stresses plus the integral of the interface stresses with respect to their corresponding domains averaged over the entire body is the same as $\boldsymbol{P}_{\mathrm{c}}$ regardless of the complexity of the stress field within the RVE domain. In view of the micro-to-macro transition, the average stress theorem motivates the macro stress to be defined by the sum of the average bulk and interface stresses as

$$
\mathrm{M}_{\boldsymbol{P}}:=\langle\boldsymbol{P}\rangle_{\mathcal{B}_{0}}+\langle\overline{\boldsymbol{P}}\rangle_{\mathcal{I}_{0}},
$$

or alternatively

$$
\mathrm{M}_{\boldsymbol{P}}:=\left\langle\widehat{\boldsymbol{b}}_{0} \otimes \boldsymbol{X}\right\rangle_{\partial \mathcal{B}_{0}} .
$$

Note, the format of the macro stress (29) is novel and the average term over the interface is independent of the jump across the interface and solely depending on the elastic response along the interface. This shall be compared with the definition of the macro deformation gradient (26) where the average term over the interface is only dependent of the jump across the interface. Clearly, in the absence of the interface contributions, i.e. $\overline{\boldsymbol{P}}=\mathbf{0}$, the relation (29) reduces to the classical average stress theorem. More interestingly, even in the presence of interfaces, the definition of the macro stress as the boundary integral (30) is identical to its format in the classical homogenization.

Hill-Mandel condition. Motivated by the extended average theorems, the macro deformation gradient ${ }^{\mathrm{M}_{\boldsymbol{F}}}$ and the macro stress ${ }^{M} \boldsymbol{P}$ are defined by relations (26) and (29), respectively. Next, we impose an incremental energy equivalence between the macro and micro scales in an extended fashion to account for the interfaces. The incremental energy equivalence between the scales is known as Hill-Mandel condition. We propose the extended Hill-Mandel condition

$$
\mathrm{M}_{\boldsymbol{P}}: \delta^{\mathrm{M}} \boldsymbol{F}-\left\langle\widehat{\boldsymbol{b}}_{0} \cdot \delta \boldsymbol{\varphi}\right\rangle_{\partial \mathcal{B}_{0}} \stackrel{!}{=} 0,
$$

or alternatively expressed as

$$
\mathrm{M}_{\boldsymbol{P}}: \delta^{\mathrm{M}} \boldsymbol{F}-\langle\boldsymbol{P}: \delta \boldsymbol{F}\rangle_{\mathcal{B}_{0}}-\langle\overline{\boldsymbol{P}}: \delta \overline{\boldsymbol{F}}\rangle_{\mathcal{I}_{0}}-\langle\{\{\boldsymbol{t}\}] \cdot \llbracket \delta \boldsymbol{\varphi} \rrbracket\rangle_{\mathcal{I}_{0}} \stackrel{!}{=} 0,
$$

whereby the alternative form is achieved using the following lemma proven in Appendix C.3.

$$
\left\langle\widehat{\boldsymbol{b}}_{0} \cdot \delta \boldsymbol{\varphi}\right\rangle_{\partial \mathcal{B}_{0}}=\langle\boldsymbol{P}: \delta \boldsymbol{F}\rangle_{\mathcal{B}_{0}}+\langle\overline{\boldsymbol{P}}: \delta \overline{\boldsymbol{F}}\rangle_{\mathcal{I}_{0}}+\langle\{\{\boldsymbol{t}\}] \cdot \llbracket \delta \boldsymbol{\varphi} \rrbracket\rangle_{\mathcal{I}_{0}} .
$$

Note, the incremental working terms on the right-hand side of Eq. (32) are dealing with the stress power within the bulk, the stress power along the interface and the traction power across the interface, respectively. The working terms 
Table 2

Summary of micro-to-macro transition accounting for general interfaces. The macroscopic quantities are expressed as integrals at the microscale. The integrals at the microscale can be written solely within the RVE (left) or over the boundary of the RVE (right). In the absence of the jump across the interface and the elastic response along the interface, the integrals reduce to their familiar formats in the classical homogenization in the absence of interfaces. Furthermore, even in the presence of general interfaces, the boundary forms of the integrals (right) remain identical to their format in the classical homogenization as long as the inclusion is entirely surrounded within the RVE and consequently, the interface does not intersect with the boundary of the RVE.

\begin{tabular}{llll}
\hline Macro deformation gradient & $\mathrm{M}_{\boldsymbol{F}}$ & $\frac{1}{\mathscr{V}_{0}} \int_{\mathcal{B}_{0}} \boldsymbol{F} \mathrm{d} V+\frac{1}{\mathscr{V}_{0}} \int_{\mathcal{I}_{0}} \llbracket \boldsymbol{\varphi} \rrbracket \otimes \overline{\boldsymbol{N}} \mathrm{d} A$ & $\frac{1}{\mathscr{V}_{0}} \int_{\partial \mathcal{B}_{0}} \boldsymbol{\varphi} \otimes \widehat{\boldsymbol{N}} \mathrm{d} A$ \\
& $\mathrm{M}_{\boldsymbol{P}}$ & $\frac{1}{\mathscr{V}_{0}} \int_{\mathcal{B}_{0}} \boldsymbol{P} \mathrm{d} V+\frac{1}{\mathscr{V}_{0}} \int_{\mathcal{I}_{0}} \overline{\boldsymbol{P}} \mathrm{d} A$ & $\frac{1}{\mathscr{V}_{0}} \int_{\partial \mathcal{B}_{0}} \widehat{\boldsymbol{b}}_{0} \otimes \boldsymbol{X} \mathrm{d} A$ \\
Macro Piola stress & $\mathrm{M}_{\boldsymbol{P}}: \delta^{\mathrm{M}} \boldsymbol{F}$ & $\frac{1}{\mathscr{V}_{0}} \int_{\mathcal{B}_{0}} \boldsymbol{P}: \delta \boldsymbol{F} \mathrm{d} V+\frac{1}{\mathscr{V}_{0}} \int_{\mathcal{I}_{0}} \overline{\boldsymbol{P}}: \delta \overline{\boldsymbol{F}} \mathrm{d} A+\frac{1}{\mathscr{V}_{0}} \int_{\mathcal{I}_{0}} \llbracket \boldsymbol{t} \boldsymbol{t} \| \delta \boldsymbol{\varphi} \rrbracket \mathrm{d} A$ & $\frac{1}{\mathscr{V}_{0}} \int_{\partial \mathcal{B}_{0}} \widehat{\boldsymbol{b}}_{0} \cdot \delta \boldsymbol{d} \mathrm{d} A$ \\
\hline
\end{tabular}

associated with the interface are due to both responses along the interface and across the interface. Recall, the macro deformation gradient does not include any term along the interface and also the macro stress is independent of the jump across the interface. Clearly, in the absence of interface contributions the extended Hill-Mandel condition (31) reduces to its classical format. Furthermore, the representation of the Hill-Mandel condition as the surface integral

$$
{ }^{\mathrm{M}} \boldsymbol{P}: \delta^{\mathrm{M}} \boldsymbol{F} \stackrel{!}{=}\left\langle\widehat{\boldsymbol{b}}_{0} \cdot \delta \boldsymbol{\varphi}\right\rangle_{\partial \mathcal{B}_{0}},
$$

is identical to its classical format regardless of interfaces. This is particularly advantageous in view of the computational implementation since one can use the well-established frameworks and subroutines developed for the classical homogenization. The same argument holds for computing the macro stress ${ }^{\mathrm{M}} \boldsymbol{P}$ according to (30) and the macro deformation gradient ${ }^{\mathrm{M}} \boldsymbol{F}$ according to (27). Table 2 summarizes the micro-to-macro transition accounting for general interfaces.

The next task is to seek suitable boundary conditions on the RVE which satisfy the extended Hill-Mandel condition (31). In order to do so, we introduce the extended Hill's identity, proven in Appendix C.4, which states that

$$
\left\langle\widehat{\boldsymbol{b}}_{0} \cdot \delta \boldsymbol{\varphi}\right\rangle_{\partial \mathcal{B}_{0}}-{ }^{\mathrm{M}} \boldsymbol{P}: \delta^{\mathrm{M}} \boldsymbol{F}=\left\langle\left[\delta \boldsymbol{\varphi}-\delta^{\mathrm{M}} \boldsymbol{F} \cdot \boldsymbol{X}\right] \cdot\left[\widehat{\boldsymbol{b}}_{0}-{ }^{\mathrm{M}} \boldsymbol{P} \cdot \widehat{\boldsymbol{N}}\right]\right\rangle_{\partial \mathcal{B}_{0}},
$$

or using lemma (32) alternatively expressed as,

$$
\langle\boldsymbol{P}: \delta \boldsymbol{F}\rangle_{\mathcal{B}_{0}}+\langle\overline{\boldsymbol{P}}: \delta \overline{\boldsymbol{F}}\rangle_{\mathcal{I}_{0}}+\langle\{\{\boldsymbol{t}\}] \cdot \llbracket \delta \boldsymbol{\varphi} \rrbracket\rangle_{\mathcal{I}_{0}}-{ }^{\mathrm{M}} \boldsymbol{P}: \delta^{\mathrm{M}} \boldsymbol{F}=\left\langle\left[\delta \boldsymbol{\varphi}-\delta^{\mathrm{M}} \boldsymbol{F} \cdot \boldsymbol{X}\right] \cdot\left[\widehat{\boldsymbol{b}}_{0}-{ }^{\mathrm{M}} \boldsymbol{P} \cdot \widehat{\boldsymbol{N}}\right]\right\rangle_{\partial \mathcal{B}_{0}} .
$$

The extended Hill's identity (33) $)_{\mathrm{a}, \mathrm{b}}$ essentially expresses the left-hand side of the Hill-Mandel condition (31) $)_{\mathrm{a}, \mathrm{b}}$ in terms of a surface integral over the external boundary of the RVE domain. This has the advantage that the extended Hill-Mandel condition, which is essentially a volume integral, can be transformed to a boundary integral and eventually identifies the appropriate boundary conditions that guarantee the incremental energy equivalence between the scales. In order to satisfy the extended Hill-Mandel condition, the right-hand side of (33)a,b should vanish. Here, we list several conditions, deduced from the right-hand side of (33) $)_{\mathrm{a}, \mathrm{b}}$ that sufficiently satisfy the extended Hill-Mandel condition (31) a,b and that agrees with the admissible boundary conditions in classical homogenization.

$$
\begin{array}{ll}
\text { Voigt } \rightsquigarrow \varphi={ }^{\mathrm{M}} \boldsymbol{F} \cdot \boldsymbol{X} & \text { in } \mathcal{B}_{0},
\end{array}
$$

linear displacement (Taylor) assumption $\Rightarrow$ Voigt bound,

$$
\mathrm{DBC} \rightsquigarrow \boldsymbol{\varphi}={ }^{\mathrm{M}} \boldsymbol{F} \cdot \boldsymbol{X} \quad \text { on } \partial \mathcal{B}_{0},
$$

linear displacement boundary condition (DBC),

$\mathrm{PBC} \rightsquigarrow\left[\boldsymbol{\varphi}-{ }^{\mathrm{M}} \boldsymbol{F} \cdot \boldsymbol{X}\right]:$ periodic, $\left[\widehat{\boldsymbol{b}}_{0}-{ }^{\mathrm{M}} \boldsymbol{P} \cdot \widehat{\boldsymbol{N}}\right]:$ anti-periodic

on $\partial \mathcal{B}_{0}$,

periodic displacement and anti-periodic traction boundary condition,

$\mathrm{TBC} \rightsquigarrow \widehat{\boldsymbol{b}}_{0}=\mathrm{M}_{\boldsymbol{P}} \cdot \widehat{\boldsymbol{N}}$

on $\partial \mathcal{B}_{0}$,

constant traction boundary condition (TBC),

Reuss $\rightsquigarrow \quad \widehat{\boldsymbol{b}}_{0}={ }^{\mathrm{M}} \boldsymbol{P} \cdot \widehat{\boldsymbol{N}}$

in $\mathcal{B}_{0}$,

constant traction (Sachs) assumption $\Rightarrow$ Reuss bound. 
Note that for periodic geometries, an anti-periodic traction $\widehat{\boldsymbol{b}}_{0}$ satisfies the anti-periodicity of $\left[\widehat{\boldsymbol{b}}_{0}-\mathrm{M}_{\boldsymbol{P}} \cdot \widehat{\boldsymbol{N}}\right] \operatorname{since}{ }^{\mathrm{M} \boldsymbol{P} \cdot \widehat{\boldsymbol{N}}}$ is anti-periodic itself due to anti-periodicity of the boundary normals. For classical homogenization, it is well-known that the Reuss condition results in the "most compliant" and the Voigt condition results in the "most stiff" response. In the remainder of this manuscript we limit ourselves to the PBC in the numerical examples. In passing, we mention that the list of admissible boundary conditions here is not exhaustive and to have a more thorough list, one shall add e.g. weakly periodic boundary conditions [58] to the list. Nonetheless, the main objective of this contribution is to establish a micro-to-macro transition scheme accounting for general imperfect interfaces and further discussion on the influence of boundary conditions is postponed to future contributions.

Remark. It can be shown that using the definitions of the macro deformation gradient (26) and the macro stress (29), all the aforementioned boundary conditions satisfy the balance of angular momentum on the macroscale [63,72].

\section{Finite element implementation}

The goal of this section is to briefly address the computational aspects of general imperfect interfaces within a finite element framework. The focus of the discussion here is on the formulation of the interfaces and in particular, in comparison to the standard framework for the bulk. In this context, it proves convenient to use a curvilinear-coordinatebased finite element methodology [116] as it mimics the underlying mathematical and geometrical concepts of the theory. For detailed exposition of the finite element formulation see for instance [117,118].

The first step towards the finite element implementation of the theory is to derive the weak form of the governing equations. In doing so, the strong form of the balance of linear momentum in the bulk (10) 1 and on the interface $(11)_{1}$ is tested (from the left) with the vector-valued test function $\delta \boldsymbol{\varphi} \in \mathcal{H}_{0}^{1}\left(\mathcal{B}_{0}\right)$ and $\delta \overline{\boldsymbol{\varphi}} \in \mathcal{H}_{0}^{1}\left(\mathcal{I}_{0}\right)$, respectively. The result is then integrated over the corresponding domains in the material configuration. By using the bulk and interface divergence theorems and the superficiality property of the interface Piola stress $\overline{\boldsymbol{P}}$ (which causes the integrals containing the curvature to vanish), together with the assumption of $\bar{\varphi}=\{\{\varphi\}\}$, the weak form of the balance of linear momentum reads

$$
\begin{aligned}
& \int_{\mathcal{B}_{0}} \boldsymbol{P}: \operatorname{Grad} \delta \boldsymbol{\varphi} \mathrm{d} V+\int_{\mathcal{I}_{0}} \overline{\boldsymbol{P}}: \overline{\operatorname{Grad}} \delta \overline{\boldsymbol{\varphi}} \mathrm{d} A+\int_{\mathcal{I}_{0}}\left\{\{\boldsymbol{t}\} \cdot \llbracket \delta \boldsymbol{\varphi} \rrbracket \mathrm{d} A-\int_{\mathcal{B}_{0}} \delta \boldsymbol{\varphi} \cdot \boldsymbol{b}_{0} \mathrm{~d} V\right. \\
& -\int_{\mathcal{I}_{0}} \delta \overline{\boldsymbol{\varphi}} \cdot \overline{\boldsymbol{b}}_{0} \mathrm{~d} A-\int_{\partial \mathcal{B}_{0}^{\mathrm{N}}} \delta \boldsymbol{\varphi} \cdot \widehat{\boldsymbol{b}}_{0} \mathrm{~d} A=0, \quad \forall \delta \boldsymbol{\varphi} \in \mathcal{H}_{0}^{1}\left(\mathcal{B}_{0}\right), \quad \forall \delta \overline{\boldsymbol{\varphi}}=\left\{\{\boldsymbol{\varphi}\} \in \mathcal{H}_{0}^{1}\left(\mathcal{I}_{0}\right),\right.
\end{aligned}
$$

in which $\partial \mathcal{B}_{0}^{\mathrm{N}}$ is the portion of the boundary subject to Neumann-type boundary condition and $\mathcal{H}_{0}^{1}$ denotes the Sobolev space of order 1 where the test functions are specified to be zero on the Dirichlet portion of the boundary. For the sake of brevity, we limit the discussion here to the finite element implementation at the microscale and therefore, the body forces $\boldsymbol{b}_{0}$ vanish. Furthermore, we neglect the interface forces $\overline{\boldsymbol{b}}_{0}$. Finally, we omit the last integral on the boundary of the domain since this part is not influenced by the interface at the microscale and is standard in all computational homogenization schemes dependent on the boundary condition imposed on the RVE. Hence, the reduced weak form reads

$$
\begin{aligned}
& \int_{\mathcal{B}_{0}} \boldsymbol{P}: \operatorname{Grad} \delta \boldsymbol{\varphi} \mathrm{d} V+\int_{\mathcal{I}_{0}} \overline{\boldsymbol{P}}: \overline{\operatorname{Grad}} \delta \overline{\boldsymbol{\varphi}} \mathrm{d} A+\int_{\mathcal{I}_{0}}\{\{\boldsymbol{t}\}] \cdot \llbracket \delta \boldsymbol{\varphi} \rrbracket \mathrm{d} A=0, \\
& \forall \delta \boldsymbol{\varphi} \in \mathcal{H}_{0}^{1}\left(\mathcal{B}_{0}\right), \quad \forall \delta \overline{\boldsymbol{\varphi}}=\{\{\boldsymbol{\varphi}\}\} \in \mathcal{H}_{0}^{1}\left(\mathcal{I}_{0}\right) .
\end{aligned}
$$

Next, the weak form (35) is discretized in space. The discretization is carried out by means of the finite element method and more specifically using the Bubnov-Galerkin scheme. To have a straightforward and efficient finite element scheme, the interface elements are chosen to be consistent with the bulk elements. That is, if the bulk is discretized using tri-quadratic elements, then bi-quadratic elements are used for the interface. This choice has the advantage that the facets of two adjacent bulk elements can be regarded as the two sides of an interface element and therefore we do not require further interpolations or hanging nodes to properly connect the interface to its surrounding bulk. The bulk and interface domains in the material configuration $\mathcal{B}_{0}$ and $\mathcal{I}_{0}$, respectively, are discretized into a set 
of bulk and interface elements as

$$
\mathcal{B}_{0} \approx \mathcal{B}_{0}^{h}=\bigwedge_{e=1}^{n \mathrm{BE}} \mathcal{B}_{0}^{e}, \quad \mathcal{I}_{0} \approx \mathcal{I}_{0}^{h}=\mathbf{A}_{e=1}^{n \mathrm{IE}} \mathcal{I}_{0}^{e},
$$

with $n \mathrm{BE}$ and $n \mathrm{IE}$ being the total number of bulk and interface elements, respectively. The geometry and the motion of the bulk and the interface are approximated as a function of the natural coordinates $\xi \in[-1,+1]^{3}$ and $\bar{\xi} \in[-1,+1]^{2}$ assigned to the bulk and the interface, respectively, using standard interpolations according to the isoparametric concept as

$$
\begin{aligned}
\left.\boldsymbol{X}\right|_{\mathcal{B}_{0}^{i}} \approx \boldsymbol{X}^{h}(\xi) & =\sum_{i=1}^{n \mathrm{NBE}} N^{i}(\xi) X^{i}, & \left.\bar{X}\right|_{\mathcal{I}_{0}^{i}} \approx \overline{\boldsymbol{X}}^{h}(\bar{\xi}) & =\sum_{i=1}^{n \text { NIE }} \bar{N}^{i}(\bar{\xi}) \bar{X}^{i}, \\
\left.\varphi\right|_{\mathcal{B}_{0}^{i}} \approx \varphi^{h}(\xi) & =\sum_{i=1}^{n \text { NBE }} N^{i}(\xi) \varphi^{i}, & \left.\bar{\varphi}\right|_{\mathcal{I}_{0}^{i}} \approx \bar{\varphi}^{h}(\bar{\xi}) & =\sum_{i=1}^{n \text { NIE }} \bar{N}^{i}(\bar{\xi}) \bar{\varphi}^{i},
\end{aligned}
$$

where $n$ NBE and $n$ NIE denote the number of nodes per bulk element and interface element, respectively. Note, the number of nodes per interface element $n$ NIE is exactly the same as the number of nodes per each facet of the bulk element and not twice that. The shape functions of the bulk and interface elements at their local node $i$ are denoted $N^{i}$ and $\bar{N}^{i}$, respectively. Since the average $\delta \bar{\varphi}=\{\{\delta \varphi\}\}$ and the jump $\llbracket \delta \varphi \rrbracket$ of the motion on the interface appear in the weak form (35), the following interpolations are particularly useful on the interface

$$
\begin{aligned}
\llbracket \varphi^{h} \rrbracket & =\sum_{i=1}^{n \mathrm{NIE}} \bar{N}^{i} \llbracket \varphi^{i} \rrbracket=\sum_{i=1}^{n \mathrm{NIE}} \bar{N}^{i}\left[\varphi^{+}\right]^{i}-\sum_{i=1}^{n \mathrm{NIE}} \bar{N}^{i}\left[\boldsymbol{\varphi}^{-}\right]^{i}, \\
\left\{\left\{\varphi^{h}\right\}\right. & =\sum_{i=1}^{n \mathrm{NIE}} \bar{N}^{i}\left\{\left\{\varphi^{i}\right\}\right\}=\frac{1}{2} \sum_{i=1}^{n \mathrm{NIE}} \bar{N}^{i}\left[\varphi^{+}\right]^{i}+\frac{1}{2} \sum_{i=1}^{n \mathrm{NIE}} \bar{N}^{i}\left[\varphi^{-}\right]^{i},
\end{aligned}
$$

where $\varphi^{+}=\left.\varphi\right|_{\mathcal{I}_{0}^{+}}$and $\varphi^{-}=\left.\varphi\right|_{\mathcal{I}_{0}^{-}}$. Applying the approximations (36)-(38) to the weak form (35) renders the fully discrete form

$$
\begin{aligned}
\underset{e=1}{\mathbf{A}_{\mathrm{BE}}} \int_{\mathcal{B}_{0}^{e}} \boldsymbol{P}:\left[\sum_{i=1}^{n \mathrm{NBE}} \delta \boldsymbol{\varphi}^{i} \otimes \operatorname{Grad} N^{i}\right] \mathrm{d} V \\
+\underset{e=1}{\mathbf{A}} \int_{\mathcal{I}_{0}^{e}} \overline{\boldsymbol{P}}:\left[\frac{1}{2} \sum_{i=1}^{n \mathrm{NIE}}\left[\delta \boldsymbol{\varphi}^{+}\right]^{i} \otimes \overline{\operatorname{Grad}} \bar{N}^{i}+\frac{1}{2} \sum_{i=1}^{n \mathrm{NIE}}\left[\boldsymbol{\varphi}^{-}\right]^{i} \otimes \overline{\operatorname{Grad}} \bar{N}^{i}\right] \mathrm{d} A \\
+\mathbf{A}_{e=1}^{n \mathrm{AE}} \int_{\mathcal{I}_{0}^{e}}\{\{\boldsymbol{t}\}] \cdot\left[\sum_{i=1}^{n \mathrm{NIE}}\left[\delta \boldsymbol{\varphi}^{+}\right]^{i} \otimes \overline{\operatorname{Grad}} \bar{N}^{i}-\sum_{i=1}^{n \mathrm{NIE}}\left[\boldsymbol{\varphi}^{-}\right]^{i} \otimes \overline{\operatorname{Grad}} \bar{N}^{i}\right] \mathrm{d} A=0 .
\end{aligned}
$$

Finally, we decompose the interface integrals into the contributions from the plus and the minus sides of the interface as

$$
\begin{aligned}
& {\underset{e}{e=1}}_{\mathrm{B} \mathrm{B}}^{\int_{\mathcal{B}_{0}^{e}}} \boldsymbol{P}:\left[\sum_{i=1}^{n \mathrm{NBE}} \delta \boldsymbol{\varphi}^{i} \otimes \operatorname{Grad} N^{i}\right] \mathrm{d} V \\
& +\underset{e=1}{n \mathrm{~A}} \int_{\mathcal{I}_{0}^{+e}} \overline{\boldsymbol{P}}:\left[\frac{1}{2} \sum_{i=1}^{n \mathrm{NIE}} \delta \boldsymbol{\varphi}^{i} \otimes \overline{\mathrm{Grad}} \bar{N}^{i}\right] \mathrm{d} A+\mathbf{A}_{e=1}^{n \mathrm{E}} \int_{\mathcal{I}_{0}^{-e}} \overline{\boldsymbol{P}}:\left[\frac{1}{2} \sum_{i=1}^{n \mathrm{NIE}} \delta \boldsymbol{\varphi}^{i} \otimes \overline{\operatorname{Grad}} \bar{N}^{i}\right] \mathrm{d} A \\
& \left.+\mathbf{A}_{e=1}^{n \mathrm{IE}} \int_{\mathcal{I}_{0}^{+e}}\{\boldsymbol{t}\}\right\} \cdot\left[\sum_{i=1}^{n \mathrm{NIE}} \delta \boldsymbol{\varphi}^{i} \otimes \overline{\operatorname{Grad}} \bar{N}^{i}\right] \mathrm{d} A-\mathbf{A}_{e=1}^{n \mathrm{IE}} \int_{\mathcal{I}_{0}^{-e}}\{\{\boldsymbol{t}\}] \cdot\left[\sum_{i=1}^{n \mathrm{NIE}} \delta \boldsymbol{\varphi}^{i} \otimes \overline{\operatorname{Grad}} \bar{N}^{i}\right] \mathrm{d} A=0 .
\end{aligned}
$$

Let $I$ denote any global node whether in the bulk or on the interface. Due to arbitrariness of the test function $\delta \varphi$, we assume that $\delta \varphi$ vanishes identically at all nodes except for the global node $I$. Note, the assembly operator gathers contributions from all elements at their local node $i$ associated with the global node $I$. This procedure furnishes the 
global nodal residual vector $\boldsymbol{R}^{I}$ at the global node $I$ as

$$
\begin{aligned}
& \boldsymbol{R}^{I}(\boldsymbol{\varphi})=\mathbf{A}_{e=1}^{n \mathrm{AE}} \int_{\mathcal{B}_{0}^{e}} \boldsymbol{P} \cdot \operatorname{Grad} N^{i} \mathrm{~d} V+\underset{e=1}{n_{\mathrm{IE}}} \int_{\mathcal{I}_{0}^{+e}} \frac{1}{2} \overline{\boldsymbol{P}} \cdot \overline{\operatorname{Grad}} \bar{N}^{i} \mathrm{~d} A+\mathbf{A}_{e=1}^{n \mathrm{E}} \int_{\mathcal{I}_{0}^{-e}} \frac{1}{2} \overline{\boldsymbol{P}} \cdot \overline{\operatorname{Grad}} \bar{N}^{i} \mathrm{~d} A \\
& +\stackrel{\mathbf{A}}{n=1}_{\mathcal{I}_{0}^{+e}}\{\{\boldsymbol{t}\}\} \cdot \overline{\mathrm{Grad}} \bar{N}^{i} \mathrm{~d} A-\mathbf{A = 1}_{\mathbf{A}^{n}}^{n \mathrm{E}} \int_{\mathcal{I}_{0}^{-e}}\{\{\boldsymbol{t}\}\} \cdot \overline{\operatorname{Grad}} \bar{N}^{i} \mathrm{~d} A \stackrel{!}{=} 0 .
\end{aligned}
$$

If we assemble all the global nodal residual vectors $\boldsymbol{R}^{I}$ into the global residual vector $[\boldsymbol{R}]$ and all the unknown motions into the global motion vector $[\varphi]$, the fully discrete nonlinear system of governing equations can be concisely stated as $[\boldsymbol{R}] \stackrel{!}{=} \mathbf{0}$ whose solution can be obtained via a Newton-Raphson scheme. The consistent linearization of the resulting system reads

$$
\begin{aligned}
& {[\boldsymbol{R}]_{k+1} \stackrel{!}{=} \mathbf{0} \Rightarrow[\boldsymbol{R}]_{k+1}=[\boldsymbol{R}]_{k}+[\boldsymbol{K}]_{k} \Delta[\boldsymbol{\varphi}]_{k} \stackrel{!}{=} \mathbf{0} \quad \text { and }} \\
& {[\boldsymbol{\varphi}]_{k+1}=[\boldsymbol{\varphi}]_{k}+\Delta[\boldsymbol{\varphi}]_{k} \quad \text { with } \quad[\boldsymbol{K}]:=\frac{\partial[\boldsymbol{R}]}{\partial[\boldsymbol{\varphi}]},}
\end{aligned}
$$

in which $[\boldsymbol{K}]$ denotes the corresponding algorithmic tangent (stiffness) and $k$ is the iteration step. The residual vector at the iteration $k$ is solely dependent on the motion vector $[\boldsymbol{\varphi}]_{k}$ and is briefly denoted as $[\boldsymbol{R}]_{k}:=[\boldsymbol{R}]\left([\boldsymbol{\varphi}]_{k}\right)$. Note, the brackets [•] around the residual vector, motion vector and tangent matrix are especially important to indicate that these quantities are vector and matrices after the assembly in the finite element procedure and correspond to the entire system. Let $n \mathrm{~N}$ denote the number of nodes of the whole finite element mesh. The residual vector $[\boldsymbol{R}]$, the motion vector $[\varphi]$ and the stiffness matrix $[\boldsymbol{K}]$ can be schematically represented as

$$
[\boldsymbol{R}]=\left[\begin{array}{c}
\boldsymbol{R}^{1} \\
\boldsymbol{R}^{2} \\
\vdots \\
\boldsymbol{R}^{I} \\
\vdots \\
\boldsymbol{R}^{n \mathrm{~N}}
\end{array}\right], \quad[\varphi]=\left[\begin{array}{c}
\varphi^{1} \\
\varphi^{2} \\
\vdots \\
\varphi^{J} \\
\vdots \\
\varphi^{n \mathrm{~N}}
\end{array}\right], \quad[\boldsymbol{K}]=\left[\begin{array}{cccccc}
\boldsymbol{K}^{11} & \boldsymbol{K}^{12} & \ldots & \boldsymbol{K}^{1 J} & \ldots & \boldsymbol{K}^{1 n \mathrm{~N}} \\
\boldsymbol{K}^{21} & \boldsymbol{K}^{22} & \ldots & \boldsymbol{K}^{2 J} & \ldots & \boldsymbol{K}^{2 n \mathrm{~N}} \\
\vdots & \vdots & \vdots & \vdots & \vdots & \vdots \\
\boldsymbol{K}^{I 1} & \boldsymbol{K}^{I 2} & \ldots & \boldsymbol{K}^{I J} & \ldots & \boldsymbol{K}^{I n \mathrm{~N}} \\
\vdots & \vdots & \vdots & \vdots & \vdots & \vdots \\
\boldsymbol{K}^{n \mathrm{~N} 1} & \boldsymbol{K}^{n \mathrm{~N} 2} & \ldots & \boldsymbol{K}^{n \mathrm{~N} J} & \ldots & \boldsymbol{K}^{n \mathrm{~N} n \mathrm{~N}}
\end{array}\right]
$$

Our next task is to establish the link between the nodal residual $\boldsymbol{R}^{I}$ or the nodal stiffness $\boldsymbol{K}^{I J}$ and the computational syntax. For the computational implementation, it is extremely helpful to decompose the residual and the stiffness into their contributions from the interface or from the bulk. To do so, we reorder the global nodal residual vector (41) as

$$
\begin{aligned}
& \boldsymbol{R}^{I}=\mathbf{R}^{I}+\overline{\mathbf{R}}_{+}^{I}+\overline{\mathbf{R}}_{-}^{I} \stackrel{!}{=} 0 \\
& \text { with } \begin{cases}\mathbf{R}^{I}=\int_{\mathcal{B}_{0}} \boldsymbol{P} \cdot \operatorname{Grad} N^{I} \mathrm{~d} V & \text { (bulk), } \\
\overline{\mathbf{R}}_{-}^{I}=\int_{\mathcal{I}_{0}} \frac{1}{2} \overline{\boldsymbol{P}} \cdot \overline{\operatorname{Grad}} \bar{N}^{I}-\{\{\boldsymbol{t}\}\} \cdot \overline{\operatorname{Grad}} \bar{N}^{I} \mathrm{~d} A & \text { (minus side-interface), } \\
\overline{\mathbf{R}}_{+}^{I}=\int_{\mathcal{I}_{0}} \frac{1}{2} \overline{\boldsymbol{P}} \cdot \overline{\operatorname{Grad}} \bar{N}^{I}+\{\{\boldsymbol{t}\}\} \cdot \overline{\mathrm{Grad}} \bar{N}^{I} \mathrm{~d} A \quad \text { (plus side-interface), }\end{cases}
\end{aligned}
$$

whereby we have omitted the assembly operator by replacing the local node $i$ at the element level with the global node $I$ and therefore, the integrals are not only over the individual elements but over the entire domain. From the structure of the residual (44), it is clear that the elements in the bulk possess the residual $\mathbf{R}^{I}$ and the residual of the interface elements consists of $\overline{\mathbf{R}}_{-}^{I}$ and $\overline{\mathbf{R}}_{+}^{I}$. Based on this view, we construct the tangent stiffnesses in the bulk and on the interface as the last step of the computational implementation. The tangent stiffness in the bulk assumes its standard form and reads

$$
\mathbf{K}^{I J}=\frac{\partial \mathbf{R}^{I}}{\partial \boldsymbol{\varphi}^{J}}=\int_{\mathcal{B}_{0}} \operatorname{Grad} N^{I} \cdot \mathbb{A} \cdot \operatorname{Grad} N^{J} \mathrm{~d} V \quad \text { with } \quad \mathbb{A}:=\frac{\partial \boldsymbol{P}}{\partial \boldsymbol{F}} .
$$


For the interface element, the residual and the tangent stiffness are slightly more involved as they both are a combination of the responses from the minus and plus sides of the interface as

$$
\overline{\mathbf{R}}^{I}=\left[\begin{array}{l}
\overline{\mathbf{R}}_{-}^{I} \\
\overline{\mathbf{R}}_{+}^{I}
\end{array}\right] \Rightarrow \quad \overline{\mathbf{K}}^{I J}=\left[\begin{array}{ll}
\overline{\mathbf{K}}_{--}^{I J} & \overline{\mathbf{K}}_{-+}^{I J} \\
\overline{\mathbf{K}}_{+-}^{I J} & \overline{\mathbf{K}}_{++}^{I J}
\end{array}\right]=\left[\begin{array}{ll}
\frac{\partial \overline{\mathbf{R}}_{-}^{I}}{\partial \boldsymbol{\varphi}_{-}^{J}} & \frac{\partial \overline{\mathbf{R}}_{-}^{I}}{\partial \boldsymbol{\varphi}_{+}^{J}} \\
\frac{\partial \overline{\mathbf{R}}_{+}^{I}}{\partial \boldsymbol{\varphi}_{-}^{J}} & \frac{\partial \overline{\mathbf{R}}_{+}^{I}}{\partial \boldsymbol{\varphi}_{+}^{J}}
\end{array}\right] \quad \text { or } \quad \overline{\mathbf{K}}_{ \pm \pm}^{I J}=\frac{\partial \overline{\mathbf{R}}_{ \pm}^{I}}{\partial \boldsymbol{\varphi}_{ \pm}^{J}},
$$

in which

$$
\overline{\mathbf{K}}_{ \pm \pm}^{I J}=\frac{\partial \overline{\mathbf{R}}_{ \pm}^{I}}{\partial \boldsymbol{\varphi}_{ \pm}^{J}}=\frac{\partial}{\partial \varphi_{ \pm}^{J}} \int_{\mathcal{I}_{0}} \frac{1}{2} \overline{\boldsymbol{P}} \cdot \overline{\operatorname{Grad}} \bar{N}^{I} \pm\{\{t\}\} \cdot \overline{\operatorname{Grad}} \bar{N}^{I} \mathrm{~d} A,
$$

can be expanded as

$$
\begin{aligned}
& \overline{\mathbf{K}}_{--}^{I J}=\int_{\mathcal{I}_{0}} \frac{1}{2} \overline{\operatorname{Grad}} \bar{N}^{I}=\left[\frac{1}{2} \frac{\partial \overline{\boldsymbol{P}}}{\partial \overline{\boldsymbol{F}}}-\frac{\partial\{\{\boldsymbol{t}\}\}}{\partial \overline{\boldsymbol{F}}}\right] \cdot \overline{\operatorname{Grad}} \bar{N}^{J}-\overline{\operatorname{Grad}} \bar{N}^{I}=\left[\frac{1}{2} \frac{\partial \overline{\boldsymbol{P}}}{\partial \llbracket \varphi \rrbracket}-\frac{\partial\{\{\boldsymbol{t}\}\}}{\partial \llbracket \varphi \rrbracket}\right] \bar{N}^{J} \mathrm{~d} A, \\
& \overline{\mathbf{K}}_{-+}^{I J}=\int_{\mathcal{I}_{0}} \frac{1}{2} \overline{\operatorname{Grad}} \bar{N}^{I}=\left[\frac{1}{2} \frac{\partial \overline{\boldsymbol{P}}}{\partial \overline{\boldsymbol{F}}}-\frac{\partial\{\{\boldsymbol{t}\}\}}{\partial \overline{\boldsymbol{F}}}\right] \cdot \overline{\operatorname{Grad}} \bar{N}^{J}+\overline{\operatorname{Grad}} \bar{N}^{I}=\left[\frac{1}{2} \frac{\partial \overline{\boldsymbol{P}}}{\partial \llbracket \varphi \rrbracket}-\frac{\partial\{\{\boldsymbol{t}\}\}}{\partial \llbracket \varphi \rrbracket}\right] \bar{N}^{J} \mathrm{~d} A, \\
& \overline{\mathbf{K}}_{+-}^{I J}=\int_{\mathcal{I}_{0}} \frac{1}{2} \overline{\operatorname{Grad}} \bar{N}^{I}=\left[\frac{1}{2} \frac{\partial \overline{\boldsymbol{P}}}{\partial \overline{\boldsymbol{F}}}+\frac{\partial\{\{\boldsymbol{t}\}\}}{\partial \overline{\boldsymbol{F}}}\right] \cdot \overline{\operatorname{Grad}} \bar{N}^{J}-\overline{\operatorname{Grad}} \bar{N}^{I}=\left[\frac{1}{2} \frac{\partial \overline{\boldsymbol{P}}}{\partial \llbracket \varphi \rrbracket}+\frac{\partial\{\{\boldsymbol{t}\}\}}{\partial \llbracket \varphi \rrbracket}\right] \bar{N}^{J} \mathrm{~d} A, \\
& \overline{\mathbf{K}}_{++}^{I J}=\int_{\mathcal{I}_{0}} \frac{1}{2} \overline{\operatorname{Grad}} \bar{N}^{I}=\left[\frac{1}{2} \frac{\partial \overline{\boldsymbol{P}}}{\partial \overline{\boldsymbol{F}}}+\frac{\partial\{\{\boldsymbol{t}\}\}}{\partial \overline{\boldsymbol{F}}}\right] \cdot \overline{\operatorname{Grad}} \bar{N}^{J}+\overline{\operatorname{Grad}}^{I}=\left[\frac{1}{2} \frac{\partial \overline{\boldsymbol{P}}}{\partial \llbracket \varphi \rrbracket}+\frac{\partial\{\{\boldsymbol{t}\}\}}{\partial \llbracket \varphi \rrbracket}\right] \bar{N}^{J} \mathrm{~d} A .
\end{aligned}
$$

Detailed derivations of the tangents on the interface (48) are given in Appendix D.

In this manuscript, it is of particular interest to have the interface stiffness (48) for a family of interfaces with additive decomposition of the interface free energy into the elastic response along the interface and the cohesive response across the interface as

$$
\bar{\psi}_{0}(\overline{\boldsymbol{F}}, \llbracket \varphi \rrbracket)=\bar{\psi}_{0}^{\|}(\overline{\boldsymbol{F}})+\bar{\psi}_{0}^{\perp}(\llbracket \varphi \rrbracket) .
$$

For the particular interface free energy density (49), the constitutive response of the interface (17) satisfies

$$
\overline{\boldsymbol{P}}=\frac{\partial \bar{\psi}_{0}}{\partial \overline{\boldsymbol{F}}} \Rightarrow \frac{\partial \overline{\boldsymbol{P}}}{\partial \llbracket \boldsymbol{\varphi} \rrbracket}=\mathbf{0} \quad \text { and } \quad\{[\boldsymbol{t}\}\}=\frac{\partial \bar{\psi}_{0}^{\perp}}{\partial \llbracket \boldsymbol{\varphi} \rrbracket} \quad \Rightarrow \quad \frac{\partial\{\boldsymbol{t}\}\}}{\partial \overline{\boldsymbol{F}}}=\mathbf{0},
$$

and therefore, the relations for the interface stiffness (48) simplify to

$$
\begin{aligned}
& \overline{\mathbf{K}}_{--}^{I J}=\int_{\mathcal{I}_{0}} \frac{1}{4} \overline{\operatorname{Grad}} \bar{N}^{I} \cdot \overline{\mathbb{A}}_{\|} \cdot \overline{\operatorname{Grad}} \bar{N}^{J}+\overline{\operatorname{Grad}} \bar{N}^{I} \cdot \overline{\mathbb{A}}_{\perp} \bar{N}^{J} \mathrm{~d} A, \\
& \overline{\mathbf{K}}_{-+}^{I J}=\int_{\mathcal{I}_{0}} \frac{1}{4} \overline{\operatorname{Grad}} \bar{N}^{I} \cdot \overline{\mathbb{A}}_{\|} \cdot \overline{\operatorname{Grad}} \bar{N}^{J}-\overline{\operatorname{Grad}} \bar{N}^{I} \cdot \overline{\mathbb{A}}_{\perp} \bar{N}^{J} \mathrm{~d} A, \\
& \overline{\mathbf{K}}_{+-}^{I J}=\int_{\mathcal{I}_{0}} \frac{1}{4} \overline{\operatorname{Grad}} \bar{N}^{I} \cdot \overline{\mathbb{A}}_{\|} \cdot \overline{\operatorname{Grad}} \bar{N}^{J}-\overline{\operatorname{Grad}} \bar{N}^{I} \cdot \overline{\mathbb{A}}_{\perp} \bar{N}^{J} \mathrm{~d} A, \\
& \overline{\mathbf{K}}_{++}^{I J}=\int_{\mathcal{I}_{0}} \frac{1}{4} \overline{\operatorname{Grad}} \bar{N}^{I} \cdot \overline{\mathbb{A}}_{\|} \cdot \overline{\operatorname{Grad}} \bar{N}^{J}+\overline{\operatorname{Grad}} \bar{N}^{I} \cdot \overline{\mathbb{A}}_{\perp} \bar{N}^{J} \mathrm{~d} A,
\end{aligned}
$$

with the fourth-order constitutive tensors $\overline{\mathbb{A}}_{\|}, \overline{\mathbb{A}}_{\perp}$ along and across the interface, respectively, defined as

$$
\overline{\mathbb{A}}_{\|}:=\frac{\partial \overline{\boldsymbol{P}}}{\partial \overline{\boldsymbol{F}}}=\frac{\partial}{\partial \overline{\boldsymbol{F}}}\left(\frac{\partial \bar{\psi}_{0}^{\|}}{\partial \overline{\boldsymbol{F}}}\right), \quad \overline{\mathbb{A}}_{\perp}:=\frac{\partial\{\boldsymbol{t} \boldsymbol{}\}}{\partial \llbracket \boldsymbol{\varphi} \rrbracket}=\frac{\partial}{\partial \llbracket \boldsymbol{} \boldsymbol{\varphi} \rrbracket}\left(\frac{\partial \bar{\psi}_{0}^{\perp}}{\partial \llbracket \boldsymbol{\varphi} \rrbracket}\right) .
$$


Table 3

Key steps to evaluate the constitutive relation at the level of quadrature points of the bulk element (left) and the interface element (right).

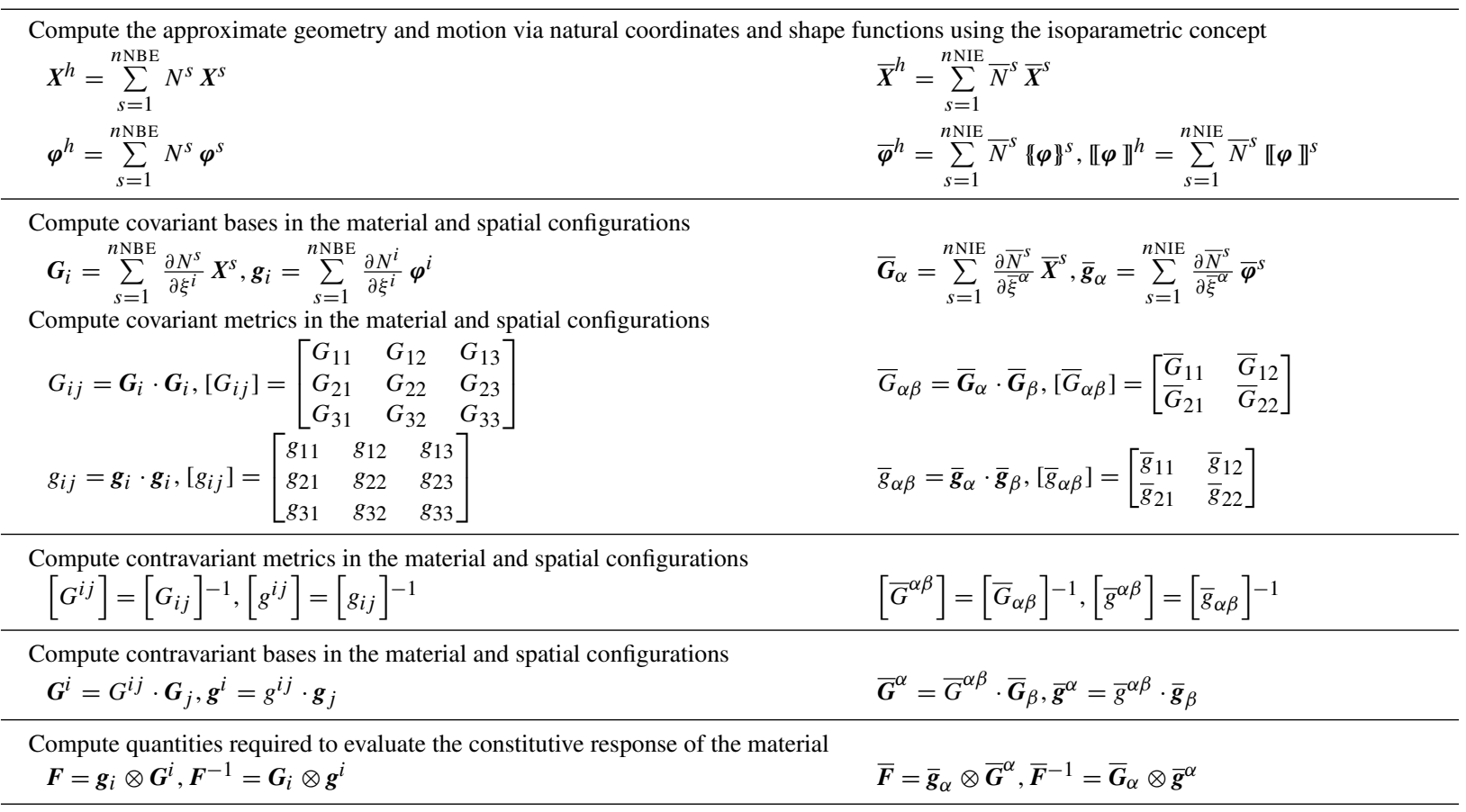

Equipped with the tangents and residuals as well as their assembly procedure presented so far, the last remaining step towards a finite element code is to evaluate the constitutive response at the element level and more specifically at the quadrature points within the elements. To pave the way for the finite element implementation, we summarize the key steps to evaluate the element response at the level of a quadrature point in the bulk and on the interface element in Table 3.

Depending on the material model of interest, further quantities may be required and can be calculated in a similar manner employing the relations and methodology presented here. For instance, if the interface normal is required, it can be easily computed via the covariant bases as

$$
\overline{\boldsymbol{N}}=\frac{\overline{\boldsymbol{G}}_{1} \times \overline{\boldsymbol{G}}_{2}}{\left|\overline{\boldsymbol{G}}_{1} \times \overline{\boldsymbol{G}}_{2}\right|} \quad \text { or } \quad \overline{\boldsymbol{n}}=\frac{\overline{\boldsymbol{g}}_{1} \times \overline{\boldsymbol{g}}_{2}}{\left|\overline{\boldsymbol{g}}_{1} \times \overline{\boldsymbol{g}}_{2}\right|} .
$$

Also, some quantities such as interface identities can be computed in several different ways and performing a consistency check such as

$$
\overline{\boldsymbol{I}}=\overline{\boldsymbol{G}}_{\alpha} \otimes \overline{\boldsymbol{G}}^{\alpha} \stackrel{?}{=} \boldsymbol{I}-\overline{\boldsymbol{N}} \otimes \overline{\boldsymbol{N}}, \quad \overline{\boldsymbol{i}}=\overline{\boldsymbol{g}}_{\alpha} \otimes \overline{\boldsymbol{g}}^{\alpha} \stackrel{?}{=} \boldsymbol{i}-\overline{\boldsymbol{n}} \otimes \overline{\boldsymbol{n}},
$$

can be reassuring.

\section{Numerical examples}

The goal of this section is to elucidate the proposed theory via a series of numerical examples. The numerical examples are chosen such that the effect of interfaces on the overall behavior is clearly observed without introducing too much complexity. Computations are carried out using our in-house finite element code which is fully functional in the three-dimensional setting. Nevertheless, we limit the numerical examples to two dimensions to better appreciate the details in the material response. The solution procedure is robust and shows the asymptotically quadratic rate of convergence associated with the Newton-Raphson scheme.

In order to proceed, we need to specify free energies for both the bulk and the interface and consequently, derive the constitutive laws. For the material response of the bulk, we assume a hyperelastic neo-Hookean free energy density in 
the material configuration

$$
\psi_{0}(\boldsymbol{F})=\frac{1}{2} \mu\left[J^{-2 / \mathrm{PD}} \boldsymbol{F}: \boldsymbol{F}-\mathrm{PD}\right]+\kappa\left[\frac{1}{4} J^{2}-\frac{1}{2} \ln J-\frac{1}{4}\right] \quad \text { with } \quad J=\operatorname{Det} \boldsymbol{F},
$$

with $\mu$ being the (first) Lamé constant and $\kappa$ the bulk modulus. This choice for energy is suitable for rubberlike materials and benefits from the original ideas of Ogden [119] to capture the macroscopic behavior of nearly incompressible soft polymers. Alternatively, it is possible to develop physically interpretable and micro-mechanically motivated material models as discussed in [120-122] among others. The free energy density (55) is general and valid for the problem dimension, PD, of two and three. In a two-dimensional setting of interest here, the free energy density and its associated constitutive response read

$$
\psi_{0}=\frac{1}{2} \mu\left[J^{-1} \boldsymbol{F}: \boldsymbol{F}-2\right]+\kappa\left[\frac{1}{4} J^{2}-\frac{1}{2} \ln J-\frac{1}{4}\right] \quad \text { and } \quad \boldsymbol{P}=\frac{\partial \psi_{0}}{\partial \boldsymbol{F}}, \quad \mathbb{A}=\frac{\partial \boldsymbol{P}}{\partial \boldsymbol{F}},
$$

with

$$
\begin{aligned}
\boldsymbol{P}= & \mu\left[\boldsymbol{F}-\frac{1}{2} \boldsymbol{F}: \boldsymbol{F} \boldsymbol{F}^{-\mathrm{t}}\right] J^{-1}+\frac{1}{2} \kappa\left[J^{2}-1\right], \\
\mathbb{A}= & \mu\left[\mathbb{I}-\frac{1}{2} \boldsymbol{F}: \boldsymbol{F} \mathbb{D}-\boldsymbol{F}^{-\mathrm{t}} \otimes \boldsymbol{F}-\boldsymbol{F} \otimes \boldsymbol{F}^{-\mathrm{t}}-\frac{1}{2} \boldsymbol{F}: \boldsymbol{F} \boldsymbol{F}^{-\mathrm{t}} \otimes \boldsymbol{F}^{-\mathrm{t}}\right] J^{-1} \\
& +\frac{1}{2} \kappa\left[\left[J^{2}-1\right] \mathbb{D}+2 J^{2} \boldsymbol{F}^{-\mathrm{t}} \otimes \boldsymbol{F}^{-\mathrm{t}}\right],
\end{aligned}
$$

in which the fourth-order tensors $\mathbb{I}$ and $\mathbb{D}$ are defined as

$$
\mathbb{I}:=\frac{\partial \boldsymbol{F}}{\partial \boldsymbol{F}}=\boldsymbol{i} \bar{\otimes} \boldsymbol{I} \quad \text { and } \quad \mathbb{D}:=\frac{\partial \boldsymbol{F}^{-\mathrm{t}}}{\partial \boldsymbol{F}}=-\boldsymbol{F}^{-\mathrm{t}} \underline{\otimes} \boldsymbol{F}^{-1} .
$$

For the material response of the interface, we additively decompose the material behavior into a tangential part along the interface and an orthogonal part across the interface. We assume a hyperelastic neo-Hookean response along the interface to capture the interface elasticity. For the orthogonal behavior of the interface, we assume the most simple and intuitive cohesive response with quadratic energy in terms of the displacement jump across the interface. See Appendix E for further details on the choice of the interface free energy. The free energy density of the interface in the material configuration is chosen as

$$
\bar{\psi}_{0}(\overline{\boldsymbol{F}}, \llbracket \boldsymbol{\varphi} \rrbracket)=\frac{1}{2} \bar{\mu}[\overline{\boldsymbol{F}}: \overline{\boldsymbol{F}}-\overline{\mathrm{PD}}-2 \ln \bar{J}]+\frac{1}{2} \bar{\lambda}\left[\frac{1}{2}\left[\bar{J}^{2}-1\right]-\ln \bar{J}\right]+\frac{1}{2} \bar{k} \llbracket \boldsymbol{\varphi} \rrbracket^{2} \quad \text { with } \quad \bar{J}=\overline{\operatorname{Det}} \overline{\boldsymbol{F}},
$$

in which $\bar{\mu}, \bar{\lambda}$ and $\bar{k}$ are the interface material parameters and $\overline{\mathrm{PD}}$ stands for the interface problem dimension. In a threedimensional setting, the interface is a two-dimensional manifold and in a two-dimensional setting a one-dimensional manifold and therefore, $\overline{\mathrm{PD}}=\mathrm{PD}-1$. For the two-dimensional setting of interest here, it is redundant to introduce two material parameters for the elastic response along the interface and hence, we set $\bar{\lambda}=0$. The free energy density and its associated constitutive response on the interface read

$$
\bar{\psi}_{0}(\overline{\boldsymbol{F}}, \llbracket \boldsymbol{\varphi} \rrbracket)=\bar{\psi}_{0}^{\|}(\overline{\boldsymbol{F}})+\bar{\psi}_{0}^{\perp}(\llbracket \boldsymbol{\varphi} \rrbracket) \quad \text { and } \quad \overline{\boldsymbol{P}}=\frac{\partial \bar{\psi}_{0}^{\|}}{\partial \overline{\boldsymbol{F}}}, \quad \overline{\mathbb{A}}_{\|}=\frac{\partial \overline{\boldsymbol{P}}}{\partial \overline{\boldsymbol{F}}}, \quad\{\{\boldsymbol{t}\})=\frac{\partial \bar{\psi}_{0}^{\|}}{\partial \llbracket \boldsymbol{\varphi} \rrbracket}, \quad \overline{\mathbb{A}}_{\perp}=\frac{\partial\{\{\boldsymbol{t}\}]}{\partial \llbracket \boldsymbol{\varphi} \rrbracket},
$$

with

$$
\begin{array}{ll}
\bar{\psi}_{0}^{\|}=\frac{1}{2} \bar{\mu}[\overline{\boldsymbol{F}}: \overline{\boldsymbol{F}}-1-2 \ln \overline{\boldsymbol{J}}], & \bar{\psi}_{0}^{\perp}=\frac{1}{2} \bar{k} \llbracket \boldsymbol{\varphi} \rrbracket^{2}, \\
\overline{\boldsymbol{P}}=\bar{\mu}\left[\overline{\boldsymbol{F}}-\overline{\boldsymbol{F}}^{-\mathrm{t}}\right], & \{\{\boldsymbol{t}\}=\bar{k} \llbracket \boldsymbol{\varphi} \rrbracket, \\
\overline{\mathbb{A}}_{\|}=\bar{\mu}[\overline{\mathbb{I}}-\overline{\mathbb{D}}], & \overline{\mathbb{A}}_{\perp}=\bar{k} \boldsymbol{i},
\end{array}
$$

in which the fourth-order tensors $\mathbb{I}$ and $\mathbb{D}$ are defined as

$$
\overline{\mathbb{I}}:=\frac{\partial \overline{\boldsymbol{F}}}{\partial \overline{\boldsymbol{F}}}=\boldsymbol{i} \bar{\otimes} \overline{\boldsymbol{I}} \quad \text { and } \quad \overline{\mathbb{D}}:=\frac{\partial \overline{\boldsymbol{F}}^{-\mathrm{t}}}{\partial \overline{\boldsymbol{F}}}=-\overline{\boldsymbol{F}}^{-\mathrm{t}} \underline{\otimes} \overline{\boldsymbol{F}}^{-1}+[\overline{\boldsymbol{n}} \otimes \overline{\boldsymbol{n}}] \bar{\otimes}\left[\overline{\boldsymbol{F}}^{-1} \cdot \overline{\boldsymbol{F}}^{-\mathrm{t}}\right] .
$$

For the following numerical studies, the material parameters for the matrix are constant throughout all the examples and are chosen as $\mu_{\text {matr. }}=8 \mathrm{~N} / \mathrm{mm}^{2}$ and $\lambda_{\text {matr. }}=18 \mathrm{~N} / \mathrm{mm}^{2}$. The material properties of the inclusion are varied such that the influence of the stiffness ratio between the inclusion and the matrix is evident. In particular, we study three different stiffness ratios incl./matr. of $0.1,1$ and 10 . The stiffness ratio incl./matr. $=0.1$ corresponds to an 

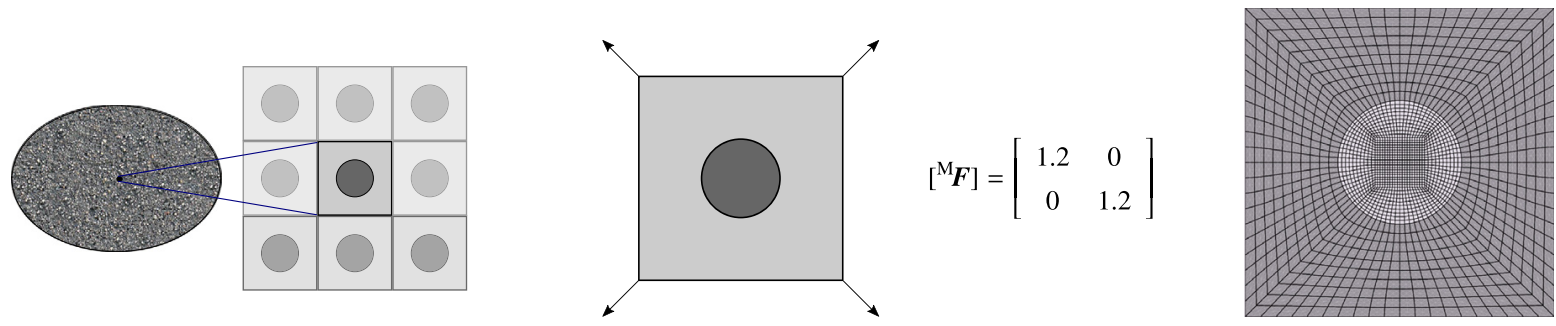

Fig. 4. Illustration of the unit-cell under volumetric expansion with the prescribed macroscopic deformation gradient ${ }^{\mathrm{M}} \boldsymbol{F}$. The micro-structure is assumed to be periodic and hence, the unit-cell being the representative volume element. The discretization of the domain using quadrilateral elements is illustrated on the right.

inclusion that is 10 times more compliant compared to the matrix. In the limit of incl./matr. $\rightarrow 0$, the microstructure resembles a porous medium. For a uniform micro-structure, the stiffness ratio equals one. In view of the classical homogenization, leaving convexity issues aside, this case is trivial as the inclusions are identical to the matrix. Nevertheless, that is not the case here as the interface results in a non-uniform and complex material behavior. Finally, the stiffness ratio incl./matr. $=10$ indicates that the inclusion is 10 times stiffer than the matrix. In the limit of incl./matr. $\rightarrow \infty$, the inclusions mimic rigid particles. We can summarize the material parameters in the following examples as

$$
\begin{aligned}
& \mu_{\text {matr. }}=8 \mathrm{~N} / \mathrm{mm}^{2} \\
& \lambda_{\text {matr. }}=18 \mathrm{~N} / \mathrm{mm}^{2}
\end{aligned} \quad \text { and } \quad\left\{\begin{array}{llll}
\text { incl. } / \text { matr. }=0.1 & \Rightarrow & \mu_{\text {incl. }}=0.8 \mathrm{~N} / \mathrm{mm}^{2}, & \lambda_{\text {incl. }}=1.8 \mathrm{~N} / \mathrm{mm}^{2}, \\
\text { incl. } / \text { matr. }=1 & \Rightarrow & \mu_{\text {incl. }}=8 \mathrm{~N} / \mathrm{mm}^{2}, & \lambda_{\text {incl. }}=18 \mathrm{~N} / \mathrm{mm}^{2}, \\
\text { incl. } / \text { matr. }=10 & \Rightarrow & \mu_{\text {incl. }}=80 \mathrm{~N} / \mathrm{mm}^{2}, & \lambda_{\text {incl. }}=180 \mathrm{~N} / \mathrm{mm}^{2} .
\end{array}\right.
$$

For the material behavior of the interface, the parameters are chosen as $\bar{\mu}=10 \mathrm{~N} / \mathrm{mm}$ and $\bar{k}=10 \mathrm{~N} / \mathrm{mm}^{3}$. Note, the ratio $\bar{\mu} / \mu$ has the dimension length but, the ratio $\bar{k} / \mu$ has the dimension length-inverse. Through the numerical examples of this section, we detail on how these parameters lead to a size effect in the material response. Clearly, the cohesive as well as the elastic interface response is the key features in the numerical results. The material parameters for the examples are chosen such that we see exaggerated interface effects. While using realistic interface parameters changes the outcomes quantitatively, the overall trends and discussions in the following examples remain valid qualitatively.

Consider a heterogeneous material, shown in Fig. 4, that consists of a periodic micro-structure such that the unitcell is the representative volume element. We prescribe a volumetric expansion of $20 \%$ on the RVE via the macroscopic deformation gradient ${ }^{\mathrm{M}} \boldsymbol{F}$ together with periodic boundary conditions on the micro-problem. The domain is discretized using 2000 bi-quadratic (Lagrange) bulk elements and 80 quadratic interface elements.

In order to study the interface effect on the material response and to appreciate the differences between the four interface models, we devise four sets of examples. The examples follow the classification summarized in Fig. 1 with the same order. Figs. 5-8 correspond to the perfect, cohesive, elastic and general interface models, respectively.

In the first set of examples, we focus on the micro-structure of Fig. 4 whereby the interface between the inclusion and the matrix behaves according to the perfect interface model. In this model both the traction jump and the displacement jump vanish and this interface model coincides with the trivial interfaces similar to the edges of the finite elements. ${ }^{7}$ This set of examples lays out the trivial interface behavior and is only given to set the stage to better understand more complex interface models proposed in this manuscript. Again, we emphasize that the perfect interface model is equivalent to no-interface model with the assumption of perfect bonding between the inclusion and the matrix. Each column in Fig. 5 corresponds to a different RVE-size. The right and left columns are 100 times larger and smaller, respectively, compared to the column in the center. Each row in Fig. 5 pertains to a different stiffness ratio between the inclusion and the matrix. The inclusion and the matrix in the middle row have the same material properties. The top and bottom rows are 10 times more compliant and stiffer, respectively, compared to the row in the

\footnotetext{
${ }^{7}$ For $C^{0}$-continuous finite elements, only the displacement is continuous at the edges of the elements and a stress jump between two adjacent elements is generally present. This stress jump is a purely numerical artifact and is irrelevant to what we denote as traction jump across the interface in this contribution. For $C^{1}$-continuous finite elements, the stress jump at the common edge of two neighboring elements vanishes identically if the common edge mimics a perfect interface model.
} 


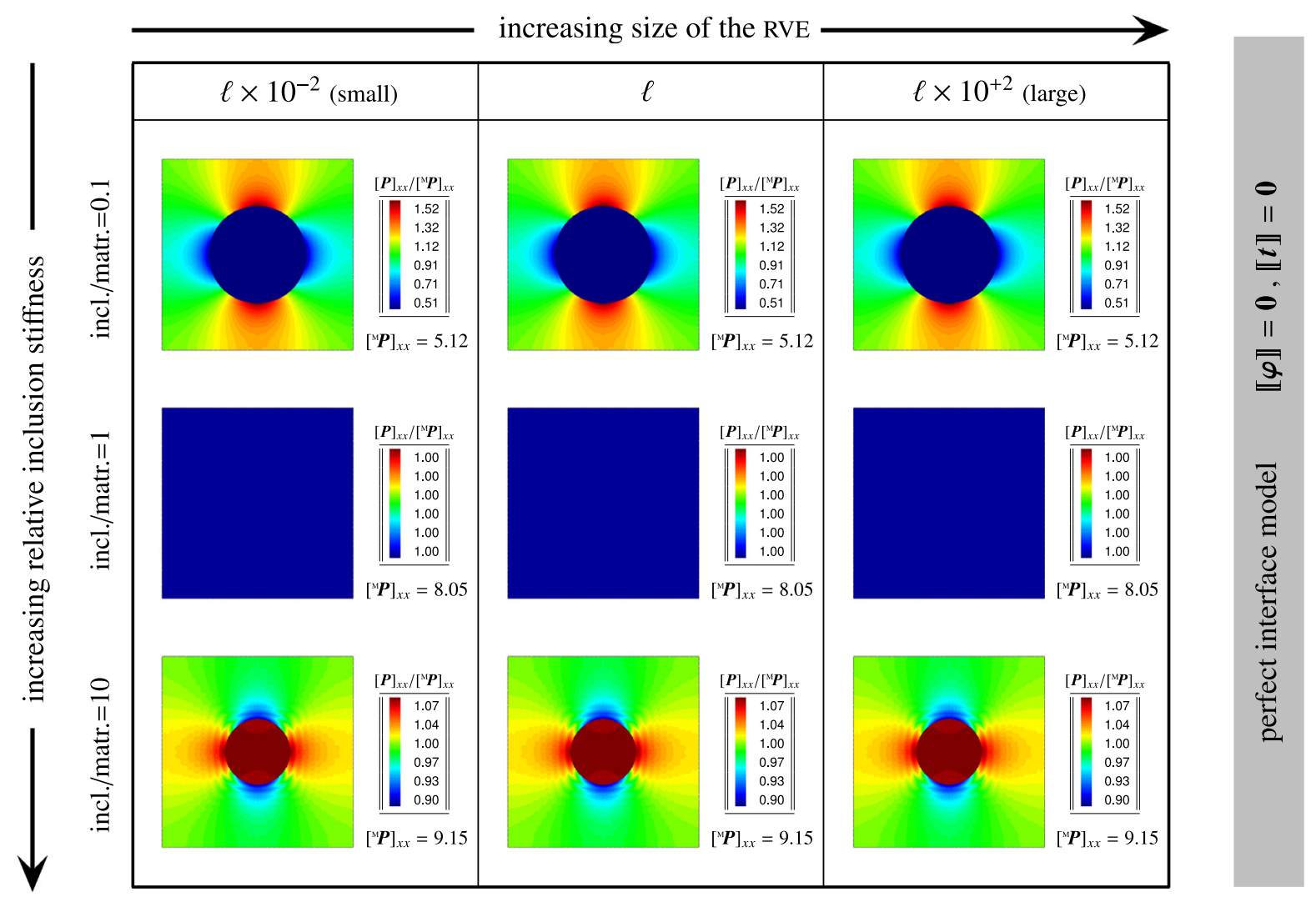

Fig. 5. Illustration of the numerical results for the RVE undergoing $20 \%$ volumetric expansion. The results correspond to three different sizes and stiffness ratios whereby the matrix properties are fixed among all the simulations. The color-map shows the distribution of the $x x$-component of the Piola stress normalized with its macroscopic counterpart given for each case. The interface model between the inclusion and the matrix is perfect and does not introduce any size effect. (For interpretation of the references to color in this figure legend, the reader is referred to the web version of this article.)

middle. The results show the distribution of the $x x$-component of the Piola stress normalized with its macroscopic counterpart given for each case. In the middle row, we observe a uniform material response and no gradient in the stress field. This is expected as the inclusion and the matrix are identical. In the first row, the inclusion undergoes more deformation but less stress compared to the matrix. This behavior is clearly the result of having an inclusion that is more compliant than the matrix. In the last row, in contrast to the first row, the inclusion experiences less deformation but more stress compared to the matrix. We can also explain this behavior as the inclusion is stiffer than the matrix. If we study Fig. 5 column-wise, we do not see any difference in the material response and hence, no size-effect. This is not surprising since the classical first-order computational homogenization lacks a physical length-scale. As we will see in the remainder of the examples, the novel interface-enhanced computational homogenization proposed in this manuscript rectifies this issue and introduces a size effect in the material behavior.

Fig. 6 deals with the same micro-structure as Fig. 5, however, the interface between the inclusion and the matrix acts as a cohesive interface model. That is, the traction jump across the interface vanishes but the displacement jump does not. According to the cohesive constitutive law (58), the average traction across the interface is proportional to the displacement jump. On the right column of Fig. 6, for a large RVE, the influence of the interface is very minor due to the small area-to-volume ratio and therefore, the overall results as well as the distribution of the stresses are very similar to the perfect interface model of Fig. 5. Decreasing the size of the RVE, that is to move column-wise towards the left, results in a more pronounced interface effect on the micro-structure response due to the increasing area-to-volume ratio. Furthermore, we observe a larger-stiffer behavior since for smaller dimensions the cohesive zone becomes more active. This can be better explained from the left column of Fig. 6 where the micro-structure resembles a porous medium and the inclusion energetically does not contribute to the material response. On the left column, 


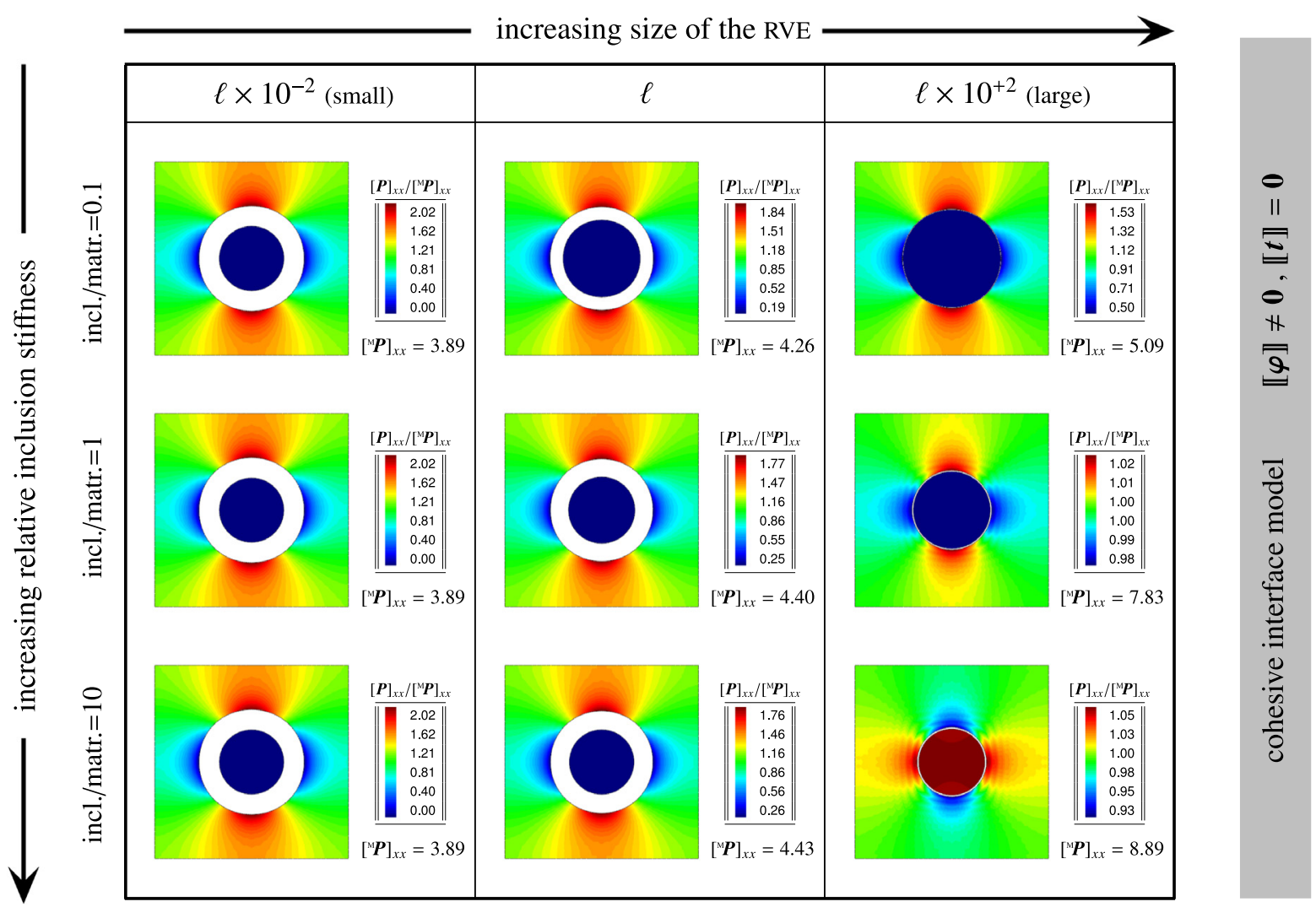

Fig. 6. Illustration of the numerical results for the RVE undergoing $20 \%$ volumetric expansion. The results correspond to three different sizes and stiffness ratios whereby the matrix properties are fixed among all the simulations. The color-map shows the distribution of the $x x$-component of the Piola stress normalized with its macroscopic counterpart given for each case. The interface model between the inclusion and the matrix is cohesive and introduces a size effect such that larger is stiffer. (For interpretation of the references to color in this figure legend, the reader is referred to the web version of this article.)

the stress in the inclusions as well as the deformation vanishes and this behavior is so strong that we observe exactly the same response independent of the stiffness ratio. Clearly, in the middle column we witness a moderate interface response between the right and the left column. The interface response in the middle column is present such that it is no longer negligible as in the right column but is also not too pronounced as in the left column and the inclusion influences the overall material response. If we study Fig. 6 row-wise, it is easily noticeable that the stiffness ratio plays no role on the left column, plays a significant role on the right column and does something in between in the middle column. This behavior is expected since on the left column the inclusion itself, and consequently its stiffness, is negligible. On the right column though, the interface plays a minor role and the overall response is dominated by the inclusion and the matrix and hence, their stiffness ratio.

For the third set of examples gathered in Fig. 7, the interface between the inclusion and the matrix is elastic and coherent. That is, the displacement is continuous across the interface but the traction jump does not necessarily vanish. Based on the chosen elastic constitutive law (58), the interface demonstrates a neo-Hookean resistance. In the context of two-dimensional examples here, this means the interface resists against the change of its length. Similar to the cohesive interface model, we observe a more pronounced interface effect on the left column due to the large area-tovolume ratio and a rather minor interface effect on the right column due to the small area-to-volume ratio. On the right column, the overall results as well as the distribution of the stresses are very similar to the perfect interface model of Fig. 5. In contrast to the cohesive interface model though, we see a smaller-stiffer behavior as for smaller dimensions the interface elasticity plays a more eminent role. This can be better explained from the left column of Fig. 7 where the micro-structure resembles the matrix with rigid inclusions. At this scale, the interface elastic resistance is so strong that it fully prevails over the inclusion and the inclusion does not contribute significantly to the material response. On 


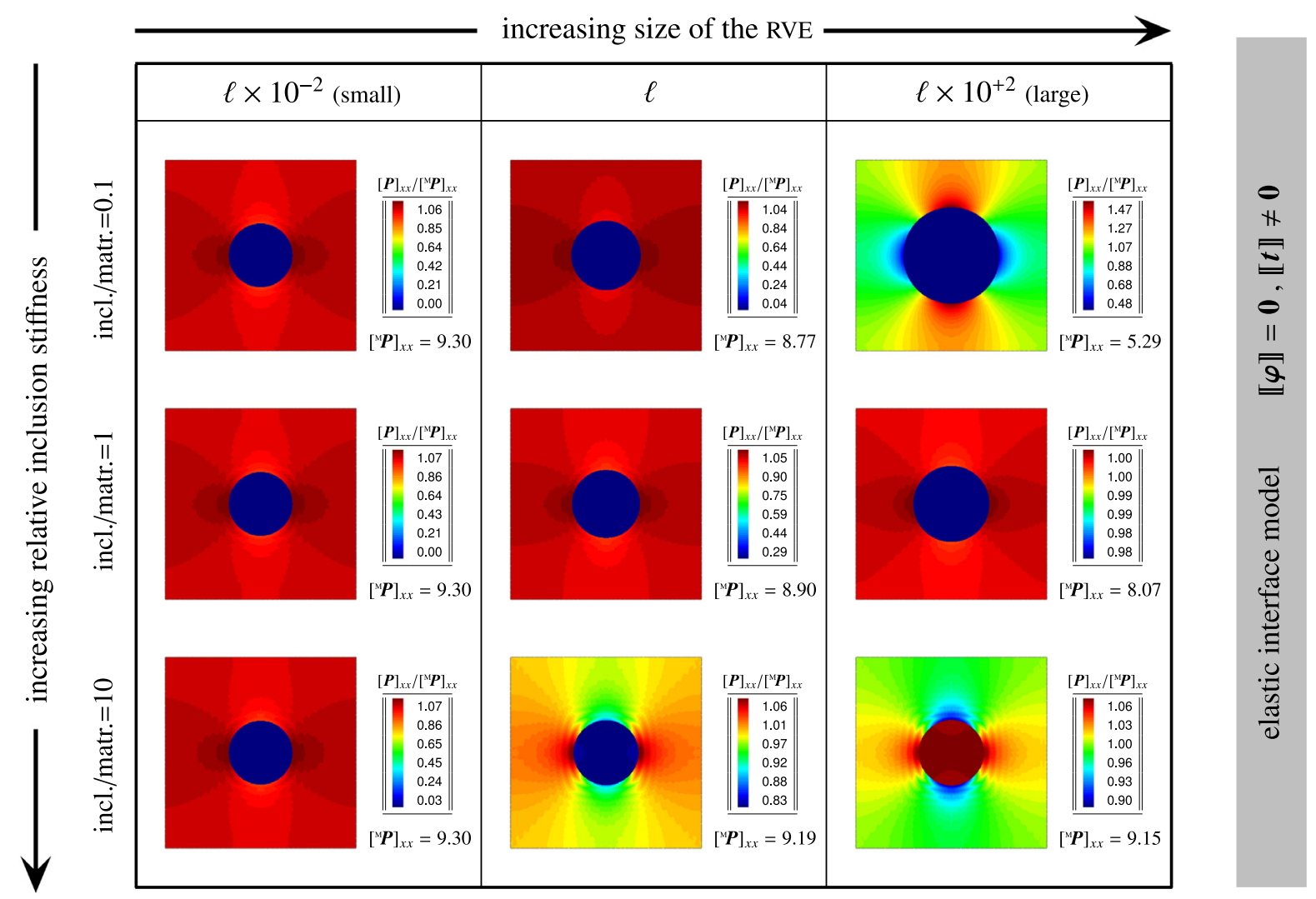

Fig. 7. Illustration of the numerical results for the RVE undergoing $20 \%$ volumetric expansion. The results correspond to three different sizes and stiffness ratios whereby the matrix properties are fixed among all the simulations. The color-map shows the distribution of the $x x$-component of the Piola stress normalized with its macroscopic counterpart given for each case. The interface model between the inclusion and the matrix is elastic and introduces a size effect such that smaller is stiffer. (For interpretation of the references to color in this figure legend, the reader is referred to the web version of this article.)

the left column, the stress in the inclusions as well as the deformation almost vanishes and this behavior is so strong that we observe exactly the same response independent of the stiffness ratio. Clearly, in the middle column we witness a moderate interface response between the right and the left column. The interface response in the middle column is present such that it is no longer negligible as in the right column and also, is not too dominant as in the left column and the inclusion affects the overall material response. If we study Fig. 7 row-wise, it is easily noticeable that the stiffness ratio plays no role on the left column, plays a significant role on the right column and does something in between in the middle column. This behavior is expected since on the left column the inclusion itself, and consequently its stiffness, is negligible. On the right column though, the interface plays a minor role and the overall response is dominated by the inclusion and the matrix and consequently, the stiffness ratio between them.

Finally, in the last set of examples in Fig. 8, the interface between the inclusion and the matrix is general imperfect in the sense that it is elastic along the interface and also cohesive across the interface. That is, neither the displacement nor the traction across the interface is continuous. According to the chosen constitutive law for the interface (58), the interface demonstrates a neo-Hookean resistance along the interface and a linear relation between the average traction and the displacement jump across the interface. This general interface model is essentially a superposition of both the elastic and the cohesive interface behavior discussed previously. Therefore, similar to the cohesive and elastic interface models, we observe a more pronounced interface effect on the left column due to the large area-to-volume ratio and a rather minor interface effect on the right column due to the small area-to-volume ratio. The overall results as well as the distribution of the stresses on the right column are very similar to the perfect interface model of Fig. 5. On one hand, we have the smaller-stiffer behavior because of the elastic response of the interface. On the other hand, due to the cohesive interface behavior, we expect a larger-stiffer behavior. Thus, the general interface behavior is more 


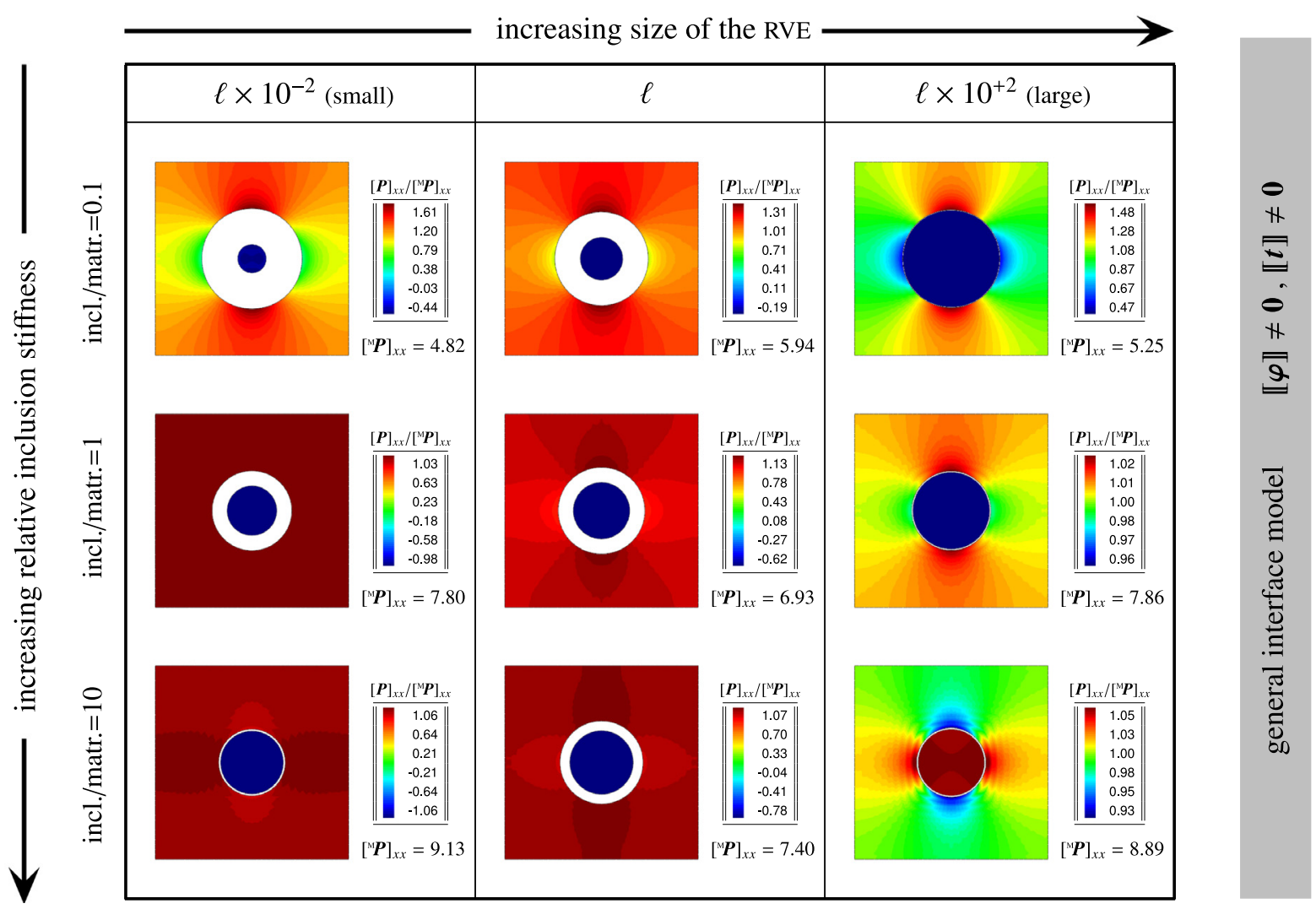

Fig. 8. Illustration of the numerical results for the RVE undergoing $20 \%$ volumetric expansion. The results correspond to three different sizes and stiffness ratios whereby the matrix properties are fixed among all the simulations. The color-map shows the distribution of the $x x$-component of the Piola stress normalized with its macroscopic counterpart given for each case. The interface model between the inclusion and the matrix is general and introduces a size effect. The size effect is a combination of both size effects due to cohesive and elastic interface models. While the cohesive contribution of the interface introduces a larger-stiffer behavior, the elastic interface response leads to a smaller-stiffer effect. Therefore, the overall response of the general interface model is complex and non-monotonic. (For interpretation of the references to color in this figure legend, the reader is referred to the web version of this article.)

complex compared to both cohesive and elastic interface models. This can be better explained by moving column-wise towards the left on Fig. 8. In contrast to both cohesive and elastic interface models, for a given row, moving between columns from right to left does not feature a uniform trend. In the first row, we see an increase and then decrease of the overall response. In the last row, we see the opposite behavior compared to the first row.

In order to fully comprehend the influence of the interface on the overall response of the micro-structure, we summarize the key features of all the previous examples in the next study. In doing so, we provide a more precise definition for the RVE-size $\ell$. Let $d$ denote a physical dimension at the micro-structure. We can use $d$ to normalize the size of the RVE, i.e. the length of the unit-cell, as $\ell / d$ illustrated in Fig. 9. Clearly, larger values for the dimensionless number $\ell / d$ correspond to a larger RVE and vice versa.

Fig. 10 gathers the macroscopic Piola stress $\left[{ }^{\mathrm{M} P}\right]_{x x}$ versus the size of the RVE. Clearly, for a volumetric expansion study here the apparent property of interest may be understood as pressure. Nevertheless, for the given micro-structure the stress $\left[{ }^{\mathrm{M}} \boldsymbol{P}\right]_{x x}$ delivers the same information as the macroscopic pressure itself. We include all the four different interface types and carry out the computations for three different stiffness ratios of $0.1,1$ and 10 . The $x$-axis on the graphs shows $\ell / d$ with $\ell$ being the length of the unit-cell. For a very large RVE, the interface effect is negligible and all classes of interfaces furnish the same response and coincide with that of the perfect interface model or equivalently the bulk-only model. The response of the perfect interface model is constant and independent of the size of the RVE, as expected. This constant is larger for the stiffness ratio of 10 (right) compared to the uniform response (center) and is expectedly smaller than the uniform response for the stiffness ratio of 0.1 (left). The cohesive interface model results in 

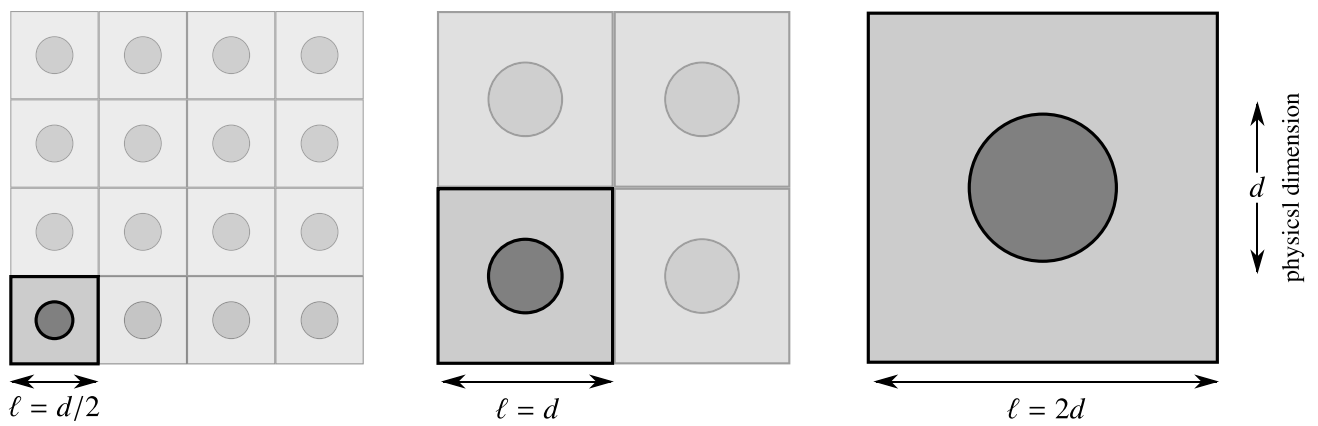

Fig. 9. Illustration of the RVE-size $\ell$. A physical dimension at the microscale is denoted $d$.
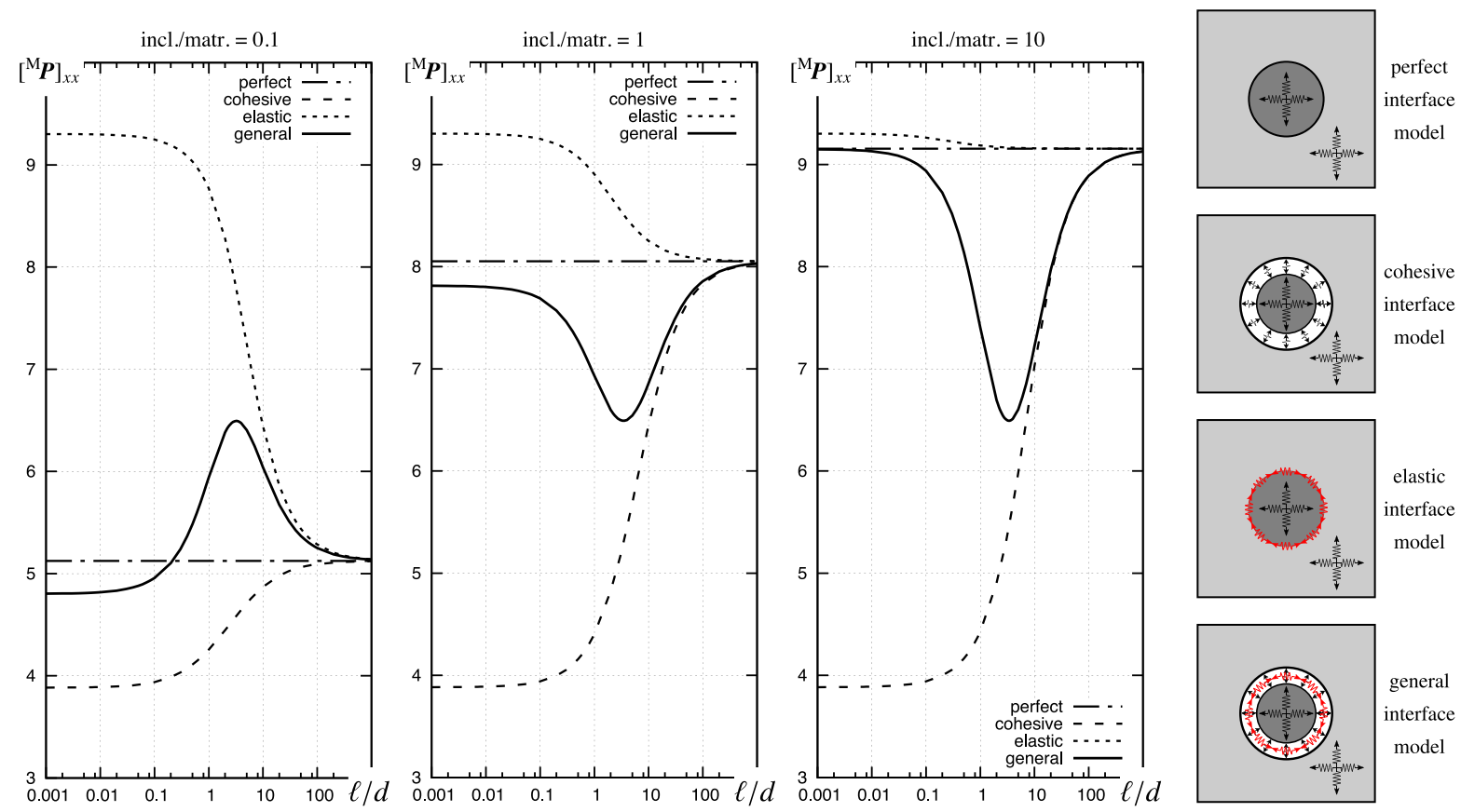

Fig. 10. Summary of the overall response of the RVE versus size for various stiffness ratios and different classes of interfaces. The graphs show the $x x$-component of the macro Piola stress versus the RVE-size normalized by a physical dimension at the micro-structure denoted $d$. The results correspond to the RVE undergoing $20 \%$ volumetric expansion. The perfect interface model does not introduce any size effect. The size effect due to the general interface model is a combination of both size effects from cohesive and elastic interface models. While the cohesive contribution of the interface introduces a larger-stiffer behavior, the elastic interface response leads to a smaller-stiffer effect. Therefore, the overall response of the general interface model is complex and non-monotonic.

a more compliant overall material behavior compared to that pertaining to the perfect interface model. The size effect due to the cohesive interface is larger-stiffer and decreasing the RVE-size leads to a more compliant response. For a very small RVE, the cohesive interface model converges to the same value for the $\left[{ }^{\mathrm{M} P}\right]_{x x}$ independent of the stiffness ratio and this value is identical to that of a porous media. In contrast to the cohesive interface model, the elastic interface model results in a stiffer overall material behavior compared to that of the perfect interface model. The size effect caused by the elastic interface is smaller-stiffer and decreasing the RVE-size leads to a stiffer response. For a very small RVE, the elastic interface model converges to the same value for the $\left[{ }^{\mathrm{M} P}\right]_{x x}$ independent of the stiffness ratio and this value is identical to that of the matrix with rigid inclusion. For the general interface model, we observe a complex and non-monotonic behavior as a result of a superposition of the cohesive and elastic interface effects. For a very small RVE, the general interface model converges to a constant value but this constant depends on the stiffness 

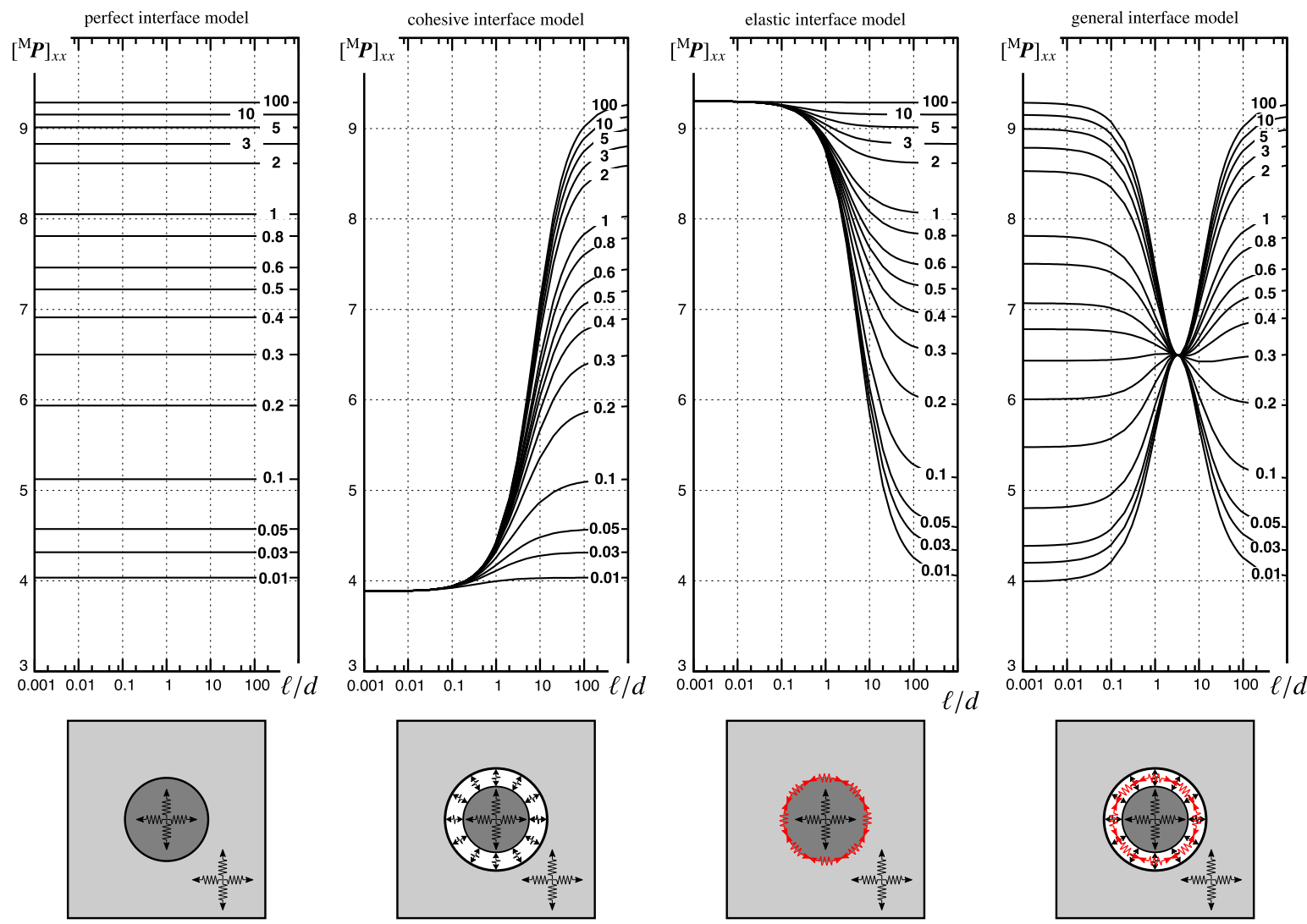

Fig. 11. Summary of the overall response of the RVE versus size for various stiffness ratios and different classes of interfaces. The number on each line indicates the stiffness ratio. The lower lines correspond to inclusions more compliant than the matrix and upper lines indicate stiffer inclusions. The graphs show the $x x$-component of the macro Piola stress versus the RVE-size normalized by a physical dimension at the micro-structure denoted $d$. The results correspond to the RVE undergoing $20 \%$ volumetric expansion. The perfect interface model does not introduce any size effect. The size effect due to the general interface model is a combination of both size effects from cohesive and elastic interface models. While the cohesive contribution of the interface introduces a larger-stiffer behavior, the elastic interface response leads to a smaller-stiffer effect. Therefore, the overall response of the general interface model is complex and non-monotonic.

ratio in contrast to the cohesive and elastic interface models. Also, the general interface model could result in both stiffer as well as more compliant overall behavior compared to the perfect interface model and eventually exhibits an extremum at a critical RVE-size where the trend of the overall response changes. Fig. 10 reveals that the size at which the extremum value is observed is identical for all the different stiffness ratios, however, the trends are not self-similar and the extremum response may correspond to a minimum or a maximum. We observe that, somewhat interestingly, not only the critical RVE-size but also the extremum value itself is identical for various stiffness ratios. To better see this rather complex material behavior, we illustrate the results of Fig. 10 in a different order and include several results associated with a broader range of stiffness ratios next. Fig. 11 shows the $x x$-component of the macro Piola stress versus $\ell / d$, however, each graph corresponds to a different interface model and contains 16 different stiffness ratios. The number on each line indicates the stiffness ratio and 100 (upper most) corresponds to a relatively rigid inclusion while 0.01 (lower most) corresponds to an extremely compliant inclusion resembling a pore-like response. As expected, the perfect interface model does not capture any size effect and it only shows the classical solution. Obviously, a stiffer inclusion results in a stiffer overall response. For all interface models, very large RVEs show the same response as the classical solution since the interface effect is rather negligible due to negligible area-to-volume ratio. In the limit of extremely small RVEs, the micro-structure with the cohesive interface model converges to a porous medium while the micro-structure with the elastic interface model converges to a matrix with rigid inclusions. The general imperfect interface model coincides with the perfect interface model in both extremes since the cohesive and elastic responses cancel each other. 


\section{Summary of key observations through numerical examples}

The aim of the computational study here is to cover various interface models and their influences on the overall response of materials and in particular, the size effect. This broad scope has led us to an unusually exhaustive numerical analysis, presented. In order to better see the connection between individual cases, we briefly summarize the key features of all the examples in what follows.

Perfect interface model does not capture any size effect. Both the displacement as well as the traction are continuous across the interface, $\llbracket \varphi \rrbracket=\mathbf{0}$ and $\llbracket t \rrbracket=\mathbf{0}$. The interface behavior can be understood as an infinitely stiff spring (rigid) normal to the interface and an infinitely compliant spring (no spring) along the interface. Reducing the size of the RVE does not influence the overall material response.

Cohesive interface model predicts a larger-stiffer size effect. The displacement suffers a jump across the interface, $\llbracket \varphi \rrbracket \neq \mathbf{0}$, but the traction remains continuous, $\llbracket t \rrbracket=\mathbf{0}$. The interface behavior can be understood as a spring (elastic) normal to the interface and an infinitely compliant spring (no spring) along the interface. Compared to the perfect interface model, it leads thus to a more compliant response and hence, to a smaller-weaker relation. Regardless of the stiffness ratio, the overall behavior converges asymptotically to a porous material response via reducing the RVE size.

Elastic interface model predicts a smaller-stiffer size effect. The displacement is continuous across the interface, $\llbracket \varphi \rrbracket=\mathbf{0}$, but the traction can suffer a jump, $\llbracket t \rrbracket \neq \mathbf{0}$. The interface behavior can be understood as an infinitely stiff spring (rigid) normal to the interface and a spring (elastic) along the interface. Compared to the perfect interface model, it leads thus to a stiffer response due to an additional spring in parallel and hence, to a smaller-stronger relation. Regardless of the stiffness ratio, the overall behavior converges asymptotically to a matrix with rigid inclusion response via reducing the RVE size.

General interface model predicts a complex size effect. Both the displacement and the traction are discontinuous across the interface, $\llbracket \varphi \rrbracket \neq \mathbf{0}$ and $\llbracket \boldsymbol{t} \rrbracket \neq \mathbf{0}$. The interface behavior can be understood as a spring (elastic) normal to the interface and a spring (elastic) along the interface. Reducing the size of the RVE in the limit can lead to both porous material as well as rigid inclusion depending on the stiffness ratio. For a specific RVE-size depending on the interface parameters, the overall material response becomes invariant with respect to the stiffness ratio.

\section{Key features and outlook}

The main objective of this contribution is to establish a micro-to-macro transition scheme accounting for general imperfect interfaces. The key features of this manuscript are:

- to formulate a thermodynamically consistent model for general imperfect interfaces,

- to incorporate such an interface model into a computational homogenization framework,

- to develop the finite element implementation of the proposed theory,

- to illustrate the influence of the proposed interface model on the overall material response via a series of numerical examples.

It is important to note that general imperfect interfaces are integrated within a computational homogenization framework for the first time here. Thus, the numerical examples are chosen such that the influence of interfaces is most pronounced and somewhat intuitive. Various extensions, out of the scope of this manuscript, remain to be added in subsequent contributions and in the near future, as follows.

From numerical studies to applications The examples here deliver only parametric studies and are purely computational. To link such numerical observations to the physics of materials, one certainly needs more information about the micro-structure itself and the nature of interface constitutive response.

Identifying interface parameters The interface material parameters can be identified using atomistic simulations or phase-field approach (see [123] for instance).

Influence of various boundary conditions Computational homogenization relies essentially on the Hill-Mandel condition which is satisfied via imposing suitable boundary conditions. Among various admissible boundary conditions, we have limited the study here to periodic boundary conditions. The influence of other boundary conditions remain to be studied.

Extension to 3D While the whole theory and the associated numerical implementation is formulated for a general three-dimensional setting, the numerical example section is limited to two-dimensional studies. This simplification has 
been made to better visualize the influence of interfaces and in order to eliminate additional complexities associated with the three-dimensional nature of the problem.

Various load cases For the numerical examples, we have limited our study to the volumetric expansion of the RVE. This choice has been made to study the interface response for a broad range of parameters such as the stiffness ratio and the size of the RVE. It is rather straightforward to prescribe extension or shear or any mixed deformation on the RVE. Nonetheless, such load cases would lead to numerical issues for certain ranges of parameters. Obviously for more realistic interface parameters, one can apply different load cases.

Comparison to analytical solutions For the generic problem of interest here, there exists no analytical solution to date. However, if certain simplifications are made, one can compare the numerical results with the corresponding analytical estimates. The numerical results here are compared against some existing analytical estimates in limit cases although the comparisons are omitted. Our next immediate plan is to establish a suitable analytical estimate for effective behavior of heterogeneous materials accounting for general imperfect interfaces and to carry out a thorough study.

Including intersections of interfaces with the RVE-boundaries The assumption that the interface does not intersect with the boundaries of the RVE has been made only for simplicity of the numerical examples. The same holds for Table 2 and its caption. However, the detailed derivations in the Appendix do include the general case in which the interface intersects with the boundary of the RVE. In order to account for such intersections, one needs to include curve integrals, too where the curves are essentially the intersections of interfaces with the boundaries of the RVE.

A fully variational micro-to-macro transition Here, we have formulated general imperfect interfaces in a thermodynamically consistent manner, but not from a variational viewpoint. The same holds for the micro-to-macro transition. More precisely, the Hill-Mandel condition is given in a heuristic and ad-hoc manner. A fully variational framework for micro-to-macro transition accounting for general imperfect interfaces remains to be established. Such a framework can give more insight to the averaging theorems presented here. For variational formulation of computational homogenization, see for instance $[63,124]$.

\section{Concluding remarks}

In the past decades, computational homogenization has proven to be a powerful strategy to compute the overall response of continua. With emerging applications of nano-objects in the last ten years, the size dependent behavior of materials has become extremely important. Nevertheless, the classical computational homogenization fails to predict the size effect and therefore, it becomes immediately inappropriate to understand the behavior of nano-materials. Here, we have presented our first attempt to capture the size effect via computational homogenization by consideration of interfaces in the micro-structure.

The commonly accepted methodology to model interfaces is to assume either the displacement or the traction to be continuous across the interface. We formulate the interface far beyond these two extreme cases and establish a thermodynamically consistent general interface model that allows for both jumps of the displacement as well as the traction. Both the cohesive zone model and interface elasticity theory are two limit cases of this general model. Next, we extend the computational homogenization to account for general interfaces. In doing so, not only suitable boundary conditions to guarantee the Hill-Mandel condition are derived but also the averaging theorems are extended to furnish meaningful averages for the micro-to-macro transition. Clearly, the proposed interface-enhanced homogenization reduces to the classical homogenization in the absence of interfaces. We have purposefully devised the entire framework such that its simplifications to the classical homogenization and more simplified interface models are evident. Finally, the proposed theory is elucidated via a series of numerical examples. Our numerical analysis clearly captures a size effect in the material response. In particular, we observe smaller-stronger and smaller-weaker for elastic and cohesive interfaces, respectively. The size effect of the general interface model is more complex and can be both smaller-stronger as well as smaller-weaker depending on other parameters.

In summary, this manuscript presents our first attempt to shed light on micro-to-macro transition accounting for general interfaces. This allows us to predict a size effect in the material response via computational homogenization. We believe that this generic framework is broadly applicable to enhance our understanding of the size dependent behavior of continua with a large variety of applications in nano-materials. 


\section{Appendix A. Geometry of interfaces}

It is enlightening to briefly review some basic terminologies and results on interfaces in the sense of twodimensional manifolds in three-dimensional space. For further details the reader is referred to [125-128] among others. A two-dimensional (smooth) surface $\mathcal{I}$ in the three dimensional, embedding Euclidean space with coordinates $\overline{\mathbb{K}}$ is parameterized by two coordinates $\eta^{\alpha}$ with $\alpha=1,2$ as $\overline{\mathrm{K}}=\overline{\mathrm{K}}\left(\eta^{\alpha}\right)$. The corresponding tangent vectors $\overline{\mathfrak{g}}_{\alpha} \in T \mathcal{I}$ to the interface coordinate lines $\eta^{\alpha}$, i.e. the covariant (natural) interface basis vectors, are given by $\overline{\mathfrak{g}}_{\alpha}=\partial_{\eta^{\alpha}} \overline{\mathrm{x}}$. The associated contravariant (dual) interface basis vectors $\overline{\mathfrak{g}}^{\alpha}$ are defined by the Kronecker property $\delta_{\beta}^{\alpha}=\overline{\mathfrak{g}}^{\alpha} \cdot \overline{\mathfrak{g}}_{\beta}$ and are explicitly related to the covariant interface basis vectors $\overline{\mathfrak{g}}_{\alpha}$ by the co- and contra-variant interface metric coefficients $\bar{g}_{\alpha \beta}$ (first fundamental form of the interface) and $\bar{g}^{\alpha \beta}$, respectively, as

$$
\overline{\mathfrak{g}}_{\alpha}=\bar{g}_{\alpha \beta} \overline{\mathbb{G}}^{\beta} \quad \text { with } \quad \bar{g}_{\alpha \beta}=\overline{\mathfrak{g}}_{\alpha} \cdot \overline{\mathfrak{g}}_{\beta}=\left[\bar{g}^{\alpha \beta}\right]^{-1}, \quad \overline{\mathfrak{g}}^{\alpha}=\bar{g}^{\alpha \beta} \overline{\mathfrak{g}}_{\beta} \quad \text { with } \quad \bar{g}^{\alpha \beta}=\overline{\mathfrak{g}}^{\alpha} \cdot \overline{\mathfrak{g}}^{\beta}=\left[\bar{g}_{\alpha \beta}\right]^{-1} .
$$

The contra- and covariant base vectors $\overline{\mathfrak{g}}^{3}$ and $\overline{\mathfrak{g}}_{3}$, normal to $T \mathcal{I}$, are defined by $\overline{\mathfrak{g}}^{3}:=\overline{\mathfrak{g}}_{1} \times \overline{\mathfrak{g}}_{2}$ and $\overline{\mathfrak{g}}_{3}:=\left[\bar{g}^{33}\right]^{-1} \overline{\mathfrak{g}}^{3}$ so that $\overline{\mathfrak{g}}^{3} \cdot \overline{\mathfrak{g}}_{3}=1$. Thereby, the corresponding contra- and covariant metric coefficients, respectively, $\left[\bar{g}^{33}\right]$ and $\left[\bar{g}_{33}\right]$ follow as

$$
\left[\bar{g}^{33}\right]=\left|\overline{\mathfrak{g}}_{1} \times \overline{\mathfrak{g}}_{2}\right|^{2}=\overline{\operatorname{det}}\left[\bar{g}_{\alpha \beta}\right]=\left[\overline{\operatorname{det}}\left[\bar{g}^{\alpha \beta}\right]\right]^{-1}=\left[\bar{g}_{33}\right]^{-1} .
$$

Accordingly, the interface area element $\mathrm{d} s$ and the interface normal $\overline{\mathrm{n}}$ are computed as

$$
\mathrm{d} s=\left|\overline{\mathfrak{g}}_{1} \times \overline{\mathfrak{g}}_{2}\right| \mathrm{d} \eta^{1} \mathrm{~d} \eta^{2}=\left[\bar{g}^{33}\right]^{1 / 2} \mathrm{~d} \eta^{1} \mathrm{~d} \eta^{2} \quad \text { and } \quad \overline{\mathfrak{n}}=\left[\bar{g}_{33}\right]^{1 / 2} \overline{\mathfrak{g}}^{3}=\left[\bar{g}^{33}\right]^{1 / 2} \overline{\mathfrak{g}}_{3} .
$$

Moreover, with $₫$ denoting the ordinary mixed-variant unit tensor of the three-dimensional embedding Euclidean space, the mixed-variant interface unit tensor $\bar{\emptyset}$ is defined as

$$
\bar{\circ}:=\delta_{\beta}^{\alpha} \overline{\mathfrak{G}}_{\alpha} \otimes \overline{\mathfrak{g}}^{\beta}=\overline{\mathfrak{g}}_{\alpha} \otimes \overline{\mathfrak{g}}^{\alpha}=\stackrel{\circ}{\mathrm{i}}-\overline{\mathfrak{g}}_{3} \otimes \overline{\mathfrak{g}}^{3}=\stackrel{\circ}{\mathrm{i}}-\overline{\mathrm{n}} \otimes \overline{\mathrm{n}} .
$$

Clearly the mixed-variant interface unit tensor acts as an interface (idempotent) projection tensor. The interface gradient and interface divergence of a vector field $\{\bullet\}$ are defined by

$$
\overline{\operatorname{grad}}\{\bullet\}:=\partial_{\eta^{\alpha}}\{\bullet\} \otimes \overline{\mathfrak{g}}^{\alpha} \quad \text { and } \quad \overline{\operatorname{div}}:=\partial_{\eta^{\alpha}}\{\bullet\} \cdot \overline{\mathfrak{g}}^{\alpha} .
$$

As a consequence, observe that $\overline{\operatorname{grad}}\{\bullet\} \cdot \overline{\mathbb{n}}=\mathbf{0}$ holds by definition. For fields that are smooth in a neighborhood of the interface, the interface gradient and interface divergence operators are alternatively defined as

$$
\overline{\operatorname{grad}}\{\bullet\}:=\operatorname{grad}\{\bullet\} \cdot \overline{\mathrm{o}} \quad \text { and } \quad \overline{\operatorname{div}}:=\overline{\operatorname{grad}}\{\bullet\}: \overline{\mathrm{o}}=\operatorname{grad}\{\bullet\}: \overline{\mathrm{o}} .
$$

Finally, the derivatives of the co- and contra-variant interface basis vectors read

$$
\partial_{\eta^{\beta}} \overline{\mathfrak{G}}_{\alpha}=\Gamma_{\alpha \beta}^{\gamma} \overline{\mathfrak{G}}_{\gamma}+\bar{k}_{\alpha \beta} \overline{\overline{\mathrm{n}}} \quad \text { and } \quad \partial_{\eta^{\beta}} \overline{\mathfrak{G}}^{\alpha}=-\Gamma_{\beta \gamma}^{\alpha} \overline{\mathfrak{G}}^{\gamma}+\bar{k}_{\beta}^{\alpha} \overline{\overline{\mathrm{n}}},
$$

where $\Gamma_{\alpha \beta}^{\gamma}=\partial_{\eta^{\beta}} \overline{\mathfrak{g}}_{\alpha} \cdot \overline{\mathfrak{g}}^{\gamma}$ denote the interface Christoffel symbols and $\bar{k}_{\alpha \beta}$ are the coefficients of the curvature tensor. The curvature tensor $\overline{\mathbb{k}}=\bar{k}_{\alpha \beta} \overline{\mathfrak{G}}^{\alpha} \otimes \overline{\mathfrak{g}}^{\beta}$ and twice the mean curvature $\bar{k}=\bar{k}_{\alpha}^{\alpha}$ of the interface $\mathcal{I}$ are defined as the negative interface gradient and interface divergence of the interface normal $\overline{\mathrm{n}}$, respectively,

$$
\overline{\mathbb{k}}:=-\overline{\operatorname{grad}} \overline{\bar{n}}=-\partial_{\eta^{\beta}} \overline{\bar{n}} \otimes \overline{\mathbb{g}}^{\beta} \quad \text { and } \quad \bar{k}:=-\overline{\operatorname{div}} \overline{\bar{n}}=-\partial_{\eta^{\beta}} \overline{\mathbb{n}} \cdot \overline{\mathfrak{g}}^{\beta} .
$$

The covariant coefficients of the curvature tensor (second fundamental form of the interface) are computed by $\bar{k}_{\alpha \beta}=\overline{\mathfrak{g}}_{\alpha} \cdot \overline{\mathbb{k}} \cdot \overline{\mathfrak{g}}_{\beta}=-\overline{\mathfrak{g}}_{\alpha} \cdot \partial_{\eta^{\beta}} \overline{\mathrm{n}}$.

\section{Appendix B. Proof of key relations regarding interfaces}

\section{B.1. Localization procedure on the interface}

In order to derive the balance of linear momentum on the interface, the global balance of linear momentum (8) is localized to an infinitesimal subdomain on the interface. This is carried out by setting the bulk domain of the cutout 
volume to vanish in the limit as

$$
\int_{\partial \mathcal{V}_{0}^{-}} \boldsymbol{v} \cdot \boldsymbol{P} \cdot \widehat{\boldsymbol{N}} \mathrm{d} A+\int_{\partial \mathcal{V}_{0}^{+}} \boldsymbol{v} \cdot \boldsymbol{P} \cdot \widehat{\boldsymbol{N}} \mathrm{d} A+\int_{\mathcal{I}_{0}} \boldsymbol{v} \cdot \overline{\boldsymbol{b}}_{0} \mathrm{~d} A+\int_{\partial \mathcal{I}_{0}} \boldsymbol{v} \cdot \overline{\boldsymbol{P}} \cdot \tilde{\boldsymbol{N}} \mathrm{d} L=0 .
$$

The boundary of the cutout volume coincides geometrically with the interface itself and therefore, $\partial \mathcal{V}_{0}^{-}=\partial \mathcal{V}_{0}^{+}=\mathcal{I}_{0}$, however, the arguments within the integrals are not identical on both sides of the interface and Eq. (B.1) reduces to

$$
\int_{\mathcal{I}_{0}} \boldsymbol{v} \cdot \boldsymbol{P}^{-} \cdot[-\overline{\boldsymbol{N}}] \mathrm{d} A+\int_{\mathcal{I}_{0}} \boldsymbol{v} \cdot \boldsymbol{P}^{+} \cdot \overline{\boldsymbol{N}} \mathrm{d} A+\int_{\mathcal{I}_{0}} \boldsymbol{v} \cdot \overline{\boldsymbol{b}}_{0} \mathrm{~d} A+\int_{\partial \mathcal{I}_{0}} \boldsymbol{v} \cdot \overline{\boldsymbol{P}} \cdot \tilde{\boldsymbol{N}} \mathrm{d} L=0 .
$$

Note, the outward normal $\widehat{N}$ to $\partial \mathcal{V}_{0}^{-}$at the limit of $\partial \mathcal{V}_{0}^{-}=\mathcal{I}_{0}$ is $[-\bar{N}]$ and also, the outward normal $\widehat{N}$ to $\partial \mathcal{V}_{0}^{+}$at the limit of $\partial \mathcal{V}_{0}^{+}=\mathcal{I}_{0}$ is $\bar{N}$. The first three terms of Eq. (B.2) are integrals over the interface and in order to transform the last term into an interface integral, we proceed with the interface divergence theorem stated below

$$
\int_{\partial \mathcal{I}_{0}}\{\overline{\boldsymbol{\bullet}}\} \cdot \tilde{\boldsymbol{N}} \mathrm{d} L=\int_{\mathcal{I}_{0}} \overline{\operatorname{Div}}\{\overline{\boldsymbol{\bullet}}\} \mathrm{d} A+\int_{\mathcal{I}_{0}} \bar{K}\{\overline{\boldsymbol{\bullet}}\} \cdot \overline{\boldsymbol{N}} \mathrm{d} A \quad \text { with } \quad \bar{K}=-\overline{\operatorname{Div}} \overline{\boldsymbol{N}} .
$$

Therefore, the last term can be written as

$$
\int_{\partial \mathcal{I}_{0}} \boldsymbol{v} \cdot \overline{\boldsymbol{P}} \cdot \tilde{\boldsymbol{N}} \mathrm{d} L=\int_{\mathcal{I}_{0}} \overline{\operatorname{Div}}(\boldsymbol{v} \cdot \overline{\boldsymbol{P}}) \mathrm{d} A+\int_{\mathcal{I}_{0}} \bar{K} \boldsymbol{v} \cdot \overline{\boldsymbol{P}} \cdot \overline{\boldsymbol{N}} \mathrm{d} A,
$$

which is then further simplified using (i) the fact that the interface stress is superficial and $\overline{\boldsymbol{P}} \cdot \overline{\boldsymbol{N}}=\mathbf{0}$ hence the second integral vanishes and (ii) employing the identity $\overline{\operatorname{Div}}(\boldsymbol{v} \cdot \overline{\boldsymbol{P}})=\overline{\operatorname{Grad} v}: \overline{\boldsymbol{P}}+\boldsymbol{v} \cdot \overline{\operatorname{Div}} \overline{\boldsymbol{P}}$ where $\overline{\mathrm{Grad}} \boldsymbol{v}=\mathbf{0}$ since the velocity $v$ is a uniformly prescribed constant. Finally we arrive at

$$
\int_{\partial \mathcal{I}_{0}} \boldsymbol{v} \cdot \overline{\boldsymbol{P}} \cdot \tilde{\boldsymbol{N}} \mathrm{d} L=\int_{\mathcal{I}_{0}} \boldsymbol{v} \cdot \overline{\operatorname{Div}} \overline{\boldsymbol{P}} \mathrm{d} A
$$

that we insert into Eq. (B.2) and obtain

$$
\int_{\mathcal{I}_{0}} \boldsymbol{v} \cdot \boldsymbol{P}^{-} \cdot[-\overline{\boldsymbol{N}}] \mathrm{d} A+\int_{\mathcal{I}_{0}} \boldsymbol{v} \cdot \boldsymbol{P}^{+} \cdot[\overline{\boldsymbol{N}}] \mathrm{d} A+\int_{\mathcal{I}_{0}} \boldsymbol{v} \cdot \overline{\boldsymbol{b}}_{0} \mathrm{~d} A+\int_{\mathcal{I}_{0}} \boldsymbol{v} \cdot \overline{\operatorname{Div}} \overline{\boldsymbol{P}} \mathrm{d} A=0,
$$

or

$$
\int_{\mathcal{I}_{0}} \boldsymbol{v} \cdot\left[\llbracket \boldsymbol{P} \rrbracket \cdot \overline{\boldsymbol{N}}+\overline{\boldsymbol{b}}_{0}+\overline{\mathrm{Div}} \overline{\boldsymbol{P}}\right] \mathrm{d} A=0
$$

that must be valid $\forall \boldsymbol{v}$. This arbitrariness property renders the local balance of linear momentum on the interface as

$$
\overline{\operatorname{Div}} \overline{\boldsymbol{P}}+\overline{\boldsymbol{b}}_{0}+\llbracket \boldsymbol{P} \rrbracket \cdot \overline{\boldsymbol{N}}=0 .
$$

Next, we proceed with localizing the global balance of angular momentum (9) on the interface. Similar to the localization of balance of linear momentum, we set the bulk domain of the cutout volume to vanish in the limit as

$$
\int_{\partial \mathcal{V}_{0}^{-}}[\omega \times \boldsymbol{x}] \cdot \boldsymbol{P} \cdot \widehat{\boldsymbol{N}} \mathrm{d} A+\int_{\partial \mathcal{V}_{0}^{+}}[\omega \times \boldsymbol{x}] \cdot \boldsymbol{P} \cdot \widehat{\boldsymbol{N}} \mathrm{d} A+\int_{\mathcal{I}_{0}}[\omega \times \overline{\boldsymbol{x}}] \cdot \overline{\boldsymbol{b}}_{0} \mathrm{~d} A+\int_{\partial \mathcal{I}_{0}}[\omega \times \overline{\boldsymbol{x}}] \cdot \overline{\boldsymbol{P}} \cdot \tilde{\boldsymbol{N}} \mathrm{d} L=0
$$

The boundary of the cutout volume coincides geometrically with the interface and $\partial \mathcal{V}_{0}^{-}=\partial \mathcal{V}_{0}^{+}=\mathcal{I}_{0}$, however, the arguments within the integrals are not identical on both sides of the interface and Eq. (B.9) reduces to

$$
\begin{aligned}
& \int_{\mathcal{I}_{0}}\left[\boldsymbol{\omega} \times \boldsymbol{x}^{-}\right] \cdot \boldsymbol{P}^{-} \cdot[-\overline{\boldsymbol{N}}] \mathrm{d} A+\int_{\mathcal{I}_{0}}\left[\boldsymbol{\omega} \times \boldsymbol{x}^{+}\right] \cdot \boldsymbol{P}^{+} \cdot \overline{\boldsymbol{N}} \mathrm{d} A+\int_{\mathcal{I}_{0}}[\boldsymbol{\omega} \times \overline{\boldsymbol{x}}] \cdot \overline{\boldsymbol{b}}_{0} \mathrm{~d} A \\
& \quad+\int_{\partial \mathcal{I}_{0}}[\boldsymbol{\omega} \times \overline{\boldsymbol{x}}] \cdot \overline{\boldsymbol{P}} \cdot \tilde{\boldsymbol{N}} \mathrm{d} L=0 .
\end{aligned}
$$


Using the interface divergence theorem (B.3), we rewrite the last term as

$$
\int_{\partial \mathcal{I}_{0}}[\omega \times \overline{\boldsymbol{x}}] \cdot \overline{\boldsymbol{P}} \cdot \tilde{\boldsymbol{N}} \mathrm{d} L=\int_{\mathcal{I}_{0}} \overline{\operatorname{Div}}([\omega \times \overline{\boldsymbol{x}}] \cdot \overline{\boldsymbol{P}}) \mathrm{d} A+\int_{\mathcal{I}_{0}} \bar{K}[\omega \times \overline{\boldsymbol{x}}] \cdot \overline{\boldsymbol{P}} \cdot \overline{\boldsymbol{N}} \mathrm{d} A,
$$

that can be simplified due to superficiality of $\overline{\boldsymbol{P}}$ as

$$
\int_{\partial \mathcal{I}_{0}}[\boldsymbol{\omega} \times \overline{\boldsymbol{x}}] \cdot \overline{\boldsymbol{P}} \cdot \tilde{\boldsymbol{N}} \mathrm{d} L=\int_{\mathcal{I}_{0}} \overline{\operatorname{Div}}([\omega \times \overline{\boldsymbol{x}}] \cdot \overline{\boldsymbol{P}}) \mathrm{d} A=\int_{\mathcal{I}_{0}} \overline{\operatorname{Grad}}(\boldsymbol{\omega} \times \overline{\boldsymbol{x}}): \overline{\boldsymbol{P}}+[\boldsymbol{\omega} \times \overline{\boldsymbol{x}}] \cdot \overline{\operatorname{Div}} \overline{\boldsymbol{P}} \mathrm{d} A .
$$

Both terms on the right-hand side can be further simplified using the identities

$$
\overline{\operatorname{Grad}}(\boldsymbol{\omega} \times \overline{\boldsymbol{x}}): \overline{\boldsymbol{P}}=\boldsymbol{\omega} \cdot\left[\boldsymbol{\varepsilon}:\left[\overline{\mathrm{Grad}} \overline{\boldsymbol{x}} \cdot \overline{\boldsymbol{P}}^{\mathrm{t}}\right]\right]=\boldsymbol{\omega} \cdot\left[\boldsymbol{\varepsilon}:\left[\overline{\boldsymbol{F}} \cdot \overline{\boldsymbol{P}}^{\mathrm{t}}\right]\right], \quad[\boldsymbol{\omega} \times \overline{\boldsymbol{x}}] \cdot \overline{\operatorname{Div}} \overline{\boldsymbol{P}}=\boldsymbol{\omega} \cdot[\overline{\boldsymbol{x}} \times \overline{\operatorname{Div}} \overline{\boldsymbol{P}}],
$$

and therefore,

$$
\int_{\partial \mathcal{I}_{0}}[\omega \times \overline{\boldsymbol{x}}] \cdot \overline{\boldsymbol{P}} \cdot \tilde{\boldsymbol{N}} \mathrm{d} L=\int_{\mathcal{I}_{0}} \omega \cdot\left[\boldsymbol{\varepsilon}:\left[\overline{\boldsymbol{F}} \cdot \overline{\boldsymbol{P}}^{\mathrm{t}}\right]\right]+\omega \cdot[\overline{\boldsymbol{x}} \times \overline{\operatorname{Div}} \overline{\boldsymbol{P}}] \mathrm{d} A .
$$

Replacing the last term of Eq. (B.10) by the results from Eq. (B.13), furnishes

$$
\begin{aligned}
& \int_{\mathcal{I}_{0}}\left[\boldsymbol{\omega} \times \boldsymbol{x}^{-}\right] \cdot \boldsymbol{P}^{-} \cdot[-\overline{\boldsymbol{N}}] \mathrm{d} A+\int_{\mathcal{I}_{0}}\left[\boldsymbol{\omega} \times \boldsymbol{x}^{+}\right] \cdot \boldsymbol{P}^{+} \cdot \overline{\boldsymbol{N}} \mathrm{d} A+\int_{\mathcal{I}_{0}}[\boldsymbol{\omega} \times \overline{\boldsymbol{x}}] \cdot \overline{\boldsymbol{b}}_{0} \mathrm{~d} A \\
& \quad+\int_{\mathcal{I}_{0}} \boldsymbol{\omega} \cdot\left[\boldsymbol{\varepsilon}:\left[\overline{\boldsymbol{F}} \cdot \overline{\boldsymbol{P}}^{\mathrm{t}}\right]\right]+\boldsymbol{\omega} \cdot[\overline{\boldsymbol{x}} \times \overline{\operatorname{Div}} \overline{\boldsymbol{P}}] \mathrm{d} A=0 .
\end{aligned}
$$

The first three terms of Eq. (B.14) can be simplified using the identity $[\boldsymbol{\omega} \times \boldsymbol{x}] \cdot\{\bullet\}=\boldsymbol{\omega} \cdot[\boldsymbol{x} \times\{\bullet\}]$ and thus

$$
\begin{aligned}
& \int_{\mathcal{I}_{0}}-\boldsymbol{\omega} \cdot\left[\boldsymbol{x}^{-} \times\left[\boldsymbol{P}^{-} \cdot \overline{\boldsymbol{N}}\right]\right]+\boldsymbol{\omega} \cdot\left[\boldsymbol{x}^{+} \times\left[\boldsymbol{P}^{+} \cdot \overline{\boldsymbol{N}}\right]\right] \mathrm{d} A+\boldsymbol{\omega} \cdot\left[\overline{\boldsymbol{x}} \times \overline{\boldsymbol{b}}_{0}\right] \mathrm{d} A \\
& \quad+\int_{\mathcal{I}_{0}} \boldsymbol{\omega} \cdot\left[\boldsymbol{\varepsilon}:\left[\overline{\boldsymbol{F}} \cdot \overline{\boldsymbol{P}}^{\mathrm{t}}\right]\right]+\boldsymbol{\omega} \cdot[\overline{\boldsymbol{x}} \times \overline{\operatorname{Div}} \overline{\boldsymbol{P}}] \mathrm{d} A=0,
\end{aligned}
$$

or alternatively

$$
\int_{\mathcal{I}_{0}} \omega \cdot\left[-\left[\boldsymbol{x}^{-} \times\left[\boldsymbol{P}^{-} \cdot \overline{\boldsymbol{N}}\right]\right]+\left[\boldsymbol{x}^{+} \times\left[\boldsymbol{P}^{+} \cdot \overline{\boldsymbol{N}}\right]\right]+\left[\overline{\boldsymbol{x}} \times \overline{\boldsymbol{b}}_{0}\right]+\boldsymbol{\varepsilon}:\left[\overline{\boldsymbol{F}} \cdot \overline{\boldsymbol{P}}^{\mathrm{t}}\right]+[\overline{\boldsymbol{x}} \times \overline{\operatorname{Div}} \overline{\boldsymbol{P}}]\right] \mathrm{d} A=0,
$$

which must hold for all $\omega$ and therefore, Eq. (B.16) results in the local balance of angular momentum on the interface

$$
-\boldsymbol{x}^{-} \times\left[\boldsymbol{P}^{-} \cdot \overline{\boldsymbol{N}}\right]+\boldsymbol{x}^{+} \times\left[\boldsymbol{P}^{+} \cdot \overline{\boldsymbol{N}}\right]+\overline{\boldsymbol{x}} \times \overline{\boldsymbol{b}}_{0}+\boldsymbol{\varepsilon}:\left[\overline{\boldsymbol{F}} \cdot \overline{\boldsymbol{P}}^{\mathrm{t}}\right]+\overline{\boldsymbol{x}} \times \overline{\operatorname{Div}} \overline{\boldsymbol{P}}=\mathbf{0} .
$$

The local balance of angular momentum (B.17) can be further simplified using the local form of the balance of linear momentum on the interface (B.8) by replacing $\overline{\operatorname{Div}} \overline{\boldsymbol{P}}+\overline{\boldsymbol{b}}_{0}$ with minus $\llbracket \boldsymbol{P} \rrbracket \cdot \overline{\boldsymbol{N}}$ and on that account

$$
-x^{-} \times\left[P^{-} \cdot \bar{N}\right]+x^{+} \times\left[P^{+} \cdot \bar{N}\right]+\varepsilon:\left[\bar{F} \cdot \bar{P}^{\mathrm{t}}\right]-\bar{x} \times[\llbracket P \rrbracket \cdot \bar{N}]=\mathbf{0},
$$

or

$$
\llbracket x \times[P \cdot \bar{N}] \rrbracket+\varepsilon:\left[\bar{F} \cdot \overline{\boldsymbol{P}}^{\mathrm{t}}\right]-\overline{\boldsymbol{x}} \times[\llbracket P \rrbracket \cdot \bar{N}]=\mathbf{0} .
$$

Using the identity $\mathbb{I}\{\bullet\} \times\{\circ\} \rrbracket=\llbracket\{\bullet\} \rrbracket \times\{\{0\}\}\}+\{\{\bullet\}\} \times \llbracket\{0\} \rrbracket$, the balance of angular momentum (B.19) reduces to

$$
\llbracket x \rrbracket \times\left\{[\boldsymbol{P} \cdot \bar{N}\}+\left\{\{x\} \times \llbracket \boldsymbol{P} \cdot \bar{N} \rrbracket+\varepsilon:\left[\bar{F} \cdot \overline{\boldsymbol{P}}^{\mathrm{t}}\right]-\overline{\boldsymbol{x}} \times[\llbracket P \rrbracket \cdot \bar{N}]=\mathbf{0},\right.\right.
$$

or alternatively

$$
\llbracket x \rrbracket \times[\{P\}] \cdot \bar{N}]+\left\{\{x\} \times[\llbracket P \rrbracket \cdot \bar{N}]+\varepsilon:\left[\bar{F} \cdot \bar{P}^{\mathrm{t}}\right]-\bar{x} \times[\llbracket P \rrbracket \cdot \bar{N}]=\mathbf{0} .\right.
$$


Recall, we assumed that the interface motion is independent of the motion of the bulk. Based on this assumption, the balance of angular momentum on the interface cannot be further simplified. However, the format of Eq. (B.21) suggests that if the interface motion is the average of the motion across the interface, i.e. if $\bar{\varphi}=\{\{\varphi\}$, then the second and the last term vanish together and we remain with a more simplified and intuitive format for balance of angular momentum as

$$
\llbracket x \rrbracket \times[\{\boldsymbol{P}\}] \cdot \bar{N}]+\varepsilon:\left[\bar{F} \cdot \overline{\boldsymbol{P}}^{\mathrm{t}}\right]=\mathbf{0} .
$$

\section{B.2. Reformulation of working}

In order to re-formulate working to its local form in the bulk, we start from the global form of working (5), omit the interface contributions and employ the classical (bulk) divergence theorem as

$$
\begin{aligned}
\mathcal{W}_{0} & =\int_{\mathcal{V}_{0}^{-}} \dot{\boldsymbol{\varphi}} \cdot \boldsymbol{b}_{0} \mathrm{~d} V+\int_{\mathcal{V}_{0}^{+}} \dot{\boldsymbol{\varphi}} \cdot \boldsymbol{b}_{0} \mathrm{~d} V+\int_{\partial \mathcal{V}_{0}^{-}} \dot{\boldsymbol{\varphi}} \cdot \boldsymbol{P} \cdot \widehat{\boldsymbol{N}} \mathrm{d} A+\int_{\partial \mathcal{V}_{0}^{+}} \dot{\boldsymbol{\varphi}} \cdot \boldsymbol{P} \cdot \widehat{\boldsymbol{N}} \mathrm{d} A \\
& =\int_{\mathcal{V}_{0}^{-}} \dot{\boldsymbol{\varphi}} \cdot \boldsymbol{b}_{0} \mathrm{~d} V+\int_{\mathcal{V}_{0}^{+}} \dot{\boldsymbol{\varphi}} \cdot \boldsymbol{b}_{0} \mathrm{~d} V+\int_{\mathcal{V}_{0}^{-}} \operatorname{Div}(\dot{\boldsymbol{\varphi}} \cdot \boldsymbol{P}) \mathrm{d} V+\int_{\mathcal{V}_{0}^{+}} \operatorname{Div}(\dot{\boldsymbol{\varphi}} \cdot \boldsymbol{P}) \mathrm{d} V,
\end{aligned}
$$

using the identity $\operatorname{Div}(\dot{\boldsymbol{\varphi}} \cdot \boldsymbol{P})=\operatorname{Grad} \dot{\boldsymbol{\varphi}}: \boldsymbol{P}+\dot{\boldsymbol{\varphi}} \cdot \operatorname{Div} \boldsymbol{P}$,

$$
\mathcal{W}_{0}=\int_{\mathcal{V}_{0}^{-}} \dot{\boldsymbol{\varphi}} \cdot \boldsymbol{b}_{0} \mathrm{~d} V+\int_{\mathcal{V}_{0}^{+}} \dot{\boldsymbol{\varphi}} \cdot \boldsymbol{b}_{0} \mathrm{~d} V+\int_{\mathcal{V}_{0}^{-}} \operatorname{Grad} \dot{\boldsymbol{\varphi}}: \boldsymbol{P}+\dot{\boldsymbol{\varphi}} \cdot \operatorname{Div} \boldsymbol{P} \mathrm{d} V+\int_{\mathcal{V}_{0}^{+}} \operatorname{Grad} \dot{\boldsymbol{\varphi}}: \boldsymbol{P}+\dot{\boldsymbol{\varphi}} \cdot \operatorname{Div} \boldsymbol{P} \mathrm{d} V,
$$

replacing $\dot{\boldsymbol{F}}=\mathrm{Grad} \dot{\boldsymbol{\varphi}}$ and reordering the terms

$$
\mathcal{W}_{0}=\int_{\mathcal{V}_{0}^{-}} \dot{\boldsymbol{\varphi}} \cdot \underbrace{\left[\boldsymbol{b}_{0}+\operatorname{Div} \boldsymbol{P}\right]}_{=\mathbf{0}} \mathrm{d} V+\int_{\mathcal{V}_{0}^{+}} \dot{\boldsymbol{\varphi}} \cdot \underbrace{\left[\boldsymbol{b}_{0}+\operatorname{Div} \boldsymbol{P}\right]}_{=\mathbf{0}} \mathrm{d} V+\int_{\mathcal{V}_{0}^{-}} \dot{\boldsymbol{F}}: \boldsymbol{P} \mathrm{d} V+\int_{\mathcal{V}_{0}^{+}} \dot{\boldsymbol{F}}: \boldsymbol{P} \mathrm{d} V,
$$

in which the first two integrals vanish due to the balance of linear momentum. Clearly, the working density in the bulk reads

$$
\mathscr{W}_{0}=\boldsymbol{P}: \dot{\boldsymbol{F}} .
$$

Next, we re-formulate working (5) to its local form on the interface by omitting the bulk terms, localize the boundary terms on the interface and using the interface divergence theorem (B.3) as

$$
\begin{aligned}
\mathcal{W}_{0} & =\int_{\partial \mathcal{V}_{0}^{-}} \dot{\boldsymbol{\varphi}} \cdot \boldsymbol{P} \cdot \widehat{\boldsymbol{N}} \mathrm{d} A+\int_{\partial \mathcal{V}_{0}^{+}} \dot{\boldsymbol{\varphi}} \cdot \boldsymbol{P} \cdot \widehat{\boldsymbol{N}} \mathrm{d} A+\int_{\mathcal{I}_{0}} \dot{\overline{\boldsymbol{\varphi}}} \cdot \overline{\boldsymbol{b}}_{0} \mathrm{~d} A+\int_{\partial \mathcal{I}_{0}} \dot{\overline{\boldsymbol{\varphi}}} \cdot \overline{\boldsymbol{P}} \cdot \widetilde{\boldsymbol{N}} \mathrm{d} L \\
& =\int_{\mathcal{I}_{0}^{-}} \dot{\boldsymbol{\varphi}}^{-} \cdot \boldsymbol{P}^{-} \cdot[-\overline{\boldsymbol{N}}] \mathrm{d} A+\int_{\mathcal{I}_{0}^{+}} \dot{\boldsymbol{\varphi}}^{+} \cdot \boldsymbol{P}^{+} \cdot \overline{\boldsymbol{N}} \mathrm{d} A+\int_{\mathcal{I}_{0}} \dot{\overline{\boldsymbol{\varphi}}} \cdot \overline{\boldsymbol{b}}_{0} \mathrm{~d} A+\int_{\partial \mathcal{I}_{0}} \overline{\operatorname{Div}}(\dot{\overline{\boldsymbol{\varphi}}} \cdot \overline{\boldsymbol{P}})+\bar{K} \dot{\overline{\boldsymbol{\varphi}}} \cdot \overline{\boldsymbol{P}} \cdot \overline{\boldsymbol{N}} \mathrm{d} A,
\end{aligned}
$$

using the identity $\overline{\operatorname{Div}}(\dot{\overline{\boldsymbol{\varphi}}} \cdot \overline{\boldsymbol{P}})=\overline{\operatorname{Grad}} \dot{\overline{\boldsymbol{\varphi}}}: \overline{\boldsymbol{P}}+\dot{\overline{\boldsymbol{\varphi}}} \cdot \overline{\operatorname{Div}} \overline{\boldsymbol{P}}$ together with $\overline{\boldsymbol{P}} \cdot \overline{\boldsymbol{N}}=\mathbf{0}$ due to the superficiality of the interface yield

$$
\mathcal{W}_{0}=\int_{\mathcal{I}_{0}^{-}} \dot{\boldsymbol{\varphi}}^{-} \cdot \boldsymbol{P}^{-} \cdot[-\overline{\boldsymbol{N}}] \mathrm{d} A+\int_{\mathcal{I}_{0}^{+}} \dot{\boldsymbol{\varphi}}^{+} \cdot \boldsymbol{P}^{+} \cdot \overline{\boldsymbol{N}} \mathrm{d} A+\int_{\mathcal{I}_{0}} \dot{\overline{\boldsymbol{\varphi}}} \cdot \overline{\boldsymbol{b}}_{0} \mathrm{~d} A+\int_{\mathcal{I}_{0}} \overline{\operatorname{Grad}} \dot{\overline{\boldsymbol{\varphi}}}: \overline{\boldsymbol{P}}+\dot{\overline{\boldsymbol{\varphi}}} \cdot \overline{\operatorname{Div}} \overline{\boldsymbol{P}} \mathrm{d} A,
$$

replacing $\dot{\overline{\boldsymbol{F}}}=\overline{\operatorname{Grad}} \dot{\overline{\boldsymbol{\varphi}}}$ and reordering the terms

$$
\begin{aligned}
\mathcal{W}_{0} & =\int_{\mathcal{I}_{0}} \llbracket \dot{\boldsymbol{\varphi}} \cdot \boldsymbol{P} \rrbracket \cdot \overline{\boldsymbol{N}} \mathrm{d} A+\int_{\mathcal{I}_{0}} \dot{\overline{\boldsymbol{\varphi}}} \cdot \underbrace{\left[\overline{\boldsymbol{b}}_{0}+\overline{\mathrm{Div}} \overline{\boldsymbol{P}}\right]}_{-\llbracket \boldsymbol{P} \rrbracket \cdot \overline{\boldsymbol{N}}}+\dot{\overline{\boldsymbol{F}}}: \overline{\boldsymbol{P}} \mathrm{d} A, \\
& =\int_{\mathcal{I}_{0}} \llbracket \dot{\boldsymbol{\varphi}} \rrbracket \cdot\{\{\boldsymbol{P}\}] \cdot \overline{\boldsymbol{N}}+\{\{\dot{\boldsymbol{\varphi}}\}] \cdot \llbracket \boldsymbol{P} \rrbracket \cdot \overline{\boldsymbol{N}}-\dot{\overline{\boldsymbol{\varphi}}} \cdot \llbracket \boldsymbol{P} \rrbracket \cdot \overline{\boldsymbol{N}}+\dot{\overline{\boldsymbol{F}}}: \overline{\boldsymbol{P}} \mathrm{d} A, \\
& =\int_{\mathcal{I}_{0}} \dot{\overline{\boldsymbol{F}}}: \overline{\boldsymbol{P}}+\llbracket \dot{\boldsymbol{\varphi}} \rrbracket \cdot\{\{\boldsymbol{P}\}] \cdot \overline{\boldsymbol{N}}+[\{\dot{\boldsymbol{\varphi}}\}-\dot{\overline{\boldsymbol{\varphi}}}] \cdot \llbracket \boldsymbol{P} \rrbracket \cdot \overline{\boldsymbol{N}} \mathrm{d} A .
\end{aligned}
$$


Clearly, the working density on the interface reads

$$
\left.\overline{\mathscr{W}}_{0}=\dot{\overline{\boldsymbol{F}}}: \overline{\boldsymbol{P}}+\llbracket \dot{\boldsymbol{\varphi}} \rrbracket \cdot\{\{\boldsymbol{P}\}] \cdot \overline{\boldsymbol{N}}+[\{\dot{\boldsymbol{\varphi}}\}\}-\dot{\bar{\varphi}}\right] \cdot \llbracket \boldsymbol{P} \rrbracket \cdot \overline{\boldsymbol{N}}
$$

Similar to the discussion on the balance of angular momentum, if we constrain the interface motion to be the average motion across the interface as $\{\{\varphi\}\}=\bar{\varphi}$, the working density on the interface $\overline{\mathscr{W}}_{0}$ simplifies to

$$
\overline{\mathscr{W}}_{0}=\dot{\overline{\boldsymbol{F}}}: \overline{\boldsymbol{P}}+\llbracket \dot{\boldsymbol{\varphi}} \rrbracket \cdot\{\{\boldsymbol{P}\} \cdot \overline{\boldsymbol{N}}+[\{\dot{\boldsymbol{\varphi}}\}\}-\dot{\bar{\varphi}}] \cdot \llbracket \boldsymbol{P} \rrbracket \cdot \overline{\boldsymbol{N}} \quad=\stackrel{\llbracket \varphi \boldsymbol{\varphi}\}=\overline{\boldsymbol{\varphi}}}{=}=\quad \overline{\mathscr{W}}_{0}=\dot{\overline{\boldsymbol{F}}}: \overline{\boldsymbol{P}}+\llbracket \dot{\boldsymbol{\varphi}} \rrbracket \cdot\{\{\boldsymbol{P}\} \cdot \overline{\boldsymbol{N}}
$$

\section{B.3. Non-standard derivations on the interface}

For the numerical implementation of interfaces within the finite element framework, certain relations and identities are required. Clearly, we do not intend to cover all the relations needed for the computational implementation. Nevertheless, we list some of the interface relations compared to their bulk counterparts as

$$
\begin{array}{ll}
\frac{\partial \bar{J}}{\partial \overline{\boldsymbol{F}}}=\bar{J} \overline{\boldsymbol{F}}^{-\mathrm{t}}, & \frac{\partial J}{\partial \boldsymbol{F}}=J \boldsymbol{F}^{-\mathrm{t}}, \\
\frac{\partial \overline{\boldsymbol{i}}}{\partial \overline{\boldsymbol{F}}}=[\overline{\boldsymbol{n}} \otimes \overline{\boldsymbol{n}}] \otimes \overline{\boldsymbol{F}}^{-\mathrm{t}}+\overline{\boldsymbol{F}}^{-\mathrm{t}} \underline{\otimes}[\overline{\boldsymbol{n}} \otimes \overline{\boldsymbol{n}}], & \frac{\partial \boldsymbol{i}}{\partial \boldsymbol{F}}=\mathbf{0} \\
\frac{\partial \overline{\boldsymbol{F}}^{-1}}{\partial \overline{\boldsymbol{F}}}=-\overline{\boldsymbol{F}}^{-1} \bar{\otimes} \overline{\boldsymbol{F}}^{-\mathrm{t}}+\left[\overline{\boldsymbol{F}}^{-1} \cdot \overline{\boldsymbol{F}}^{-\mathrm{t}}\right] \underline{\otimes}[\overline{\boldsymbol{n}} \otimes \overline{\boldsymbol{n}}], & \frac{\partial \boldsymbol{F}^{-1}}{\partial \boldsymbol{F}}=-\boldsymbol{F}^{-1} \bar{\otimes} \boldsymbol{F}^{-\mathrm{t}}, \\
\frac{\partial \overline{\boldsymbol{F}}}{\partial \overline{\mathrm{F}}}=-\overline{\boldsymbol{F}}^{-\mathrm{t}} \underline{\otimes} \overline{\boldsymbol{F}}^{-1}+[\overline{\boldsymbol{n}} \otimes \overline{\boldsymbol{n}}] \bar{\otimes}\left[\overline{\boldsymbol{F}}^{-1} \cdot \overline{\boldsymbol{F}}^{-\mathrm{t}}\right], & \frac{\partial \boldsymbol{F}^{-\mathrm{t}}}{\partial \boldsymbol{F}}=-\boldsymbol{F}^{-\mathrm{t}} \underline{\otimes \boldsymbol{F}^{-1}} .
\end{array}
$$

The aforementioned relations are extremely helpful and the linearization procedure associated with the Newton-Raphson scheme often involves them either explicitly or implicitly. The relations for the bulk (right) are standard and we do not prove them. The relations on the interface (left) are non-standard and clearly not intuitively related to their bulk counterparts. The procedure to derive the interface relations is formally similar to that for the bulk with one distinct feature that the interface identity $\bar{i}$ is not invariant with respect to the deformation. That is

$$
\frac{\partial \boldsymbol{i}}{\partial \boldsymbol{F}}=\mathbf{0} \quad \text { but } \quad \frac{\partial \overline{\boldsymbol{i}}}{\partial \overline{\boldsymbol{F}}} \neq \mathbf{0} \quad \text { since } \quad \frac{\partial \overline{\boldsymbol{i}}}{\partial \overline{\boldsymbol{F}}}=\frac{\partial(\boldsymbol{i}-\overline{\boldsymbol{n}} \otimes \overline{\boldsymbol{n}})}{\partial \overline{\boldsymbol{F}}}=-\frac{\partial(\overline{\boldsymbol{n}} \otimes \overline{\boldsymbol{n}})}{\partial \overline{\boldsymbol{F}}} .
$$

Furthermore, the inverse of the interface deformation gradient $\overline{\boldsymbol{F}}^{-1}$ is related to the inverse of the bulk deformation gradient $\boldsymbol{F}^{-1}$ via the projection $\overline{\boldsymbol{F}}^{-1}=\overline{\boldsymbol{i}} \cdot \boldsymbol{F}^{-1}$. Therefore, the derivation procedures of various relations on the interface repeatedly boil down to carry out the derivation of $\partial \overline{\boldsymbol{n}} / \partial \overline{\boldsymbol{F}}$ which is elaborated in what follows.

In order to derive $\partial \overline{\boldsymbol{n}} / \partial \overline{\boldsymbol{F}}$, first we note that the normal $\overline{\boldsymbol{n}}$ can be obtained through the vector product of the interface basis $\overline{\boldsymbol{g}}_{1}$ and $\overline{\boldsymbol{g}}_{2}$ in the spatial configuration as

$$
\overline{\boldsymbol{n}}=\frac{\overline{\boldsymbol{g}}_{1} \times \overline{\boldsymbol{g}}_{2}}{\left|\overline{\boldsymbol{g}}_{1} \times \overline{\boldsymbol{g}}_{2}\right|} \quad \quad \text { with } \quad \overline{\boldsymbol{g}}_{1}=\overline{\boldsymbol{F}} \cdot \overline{\boldsymbol{G}}_{1}, \quad \overline{\boldsymbol{g}}_{2}=\overline{\boldsymbol{F}} \cdot \overline{\boldsymbol{G}}_{2}
$$

and since $\overline{\boldsymbol{g}}_{\alpha}=\overline{\boldsymbol{F}} \cdot \overline{\boldsymbol{G}}_{\alpha}=\boldsymbol{F} \cdot \overline{\boldsymbol{I}} \cdot \overline{\boldsymbol{G}}_{\alpha}=\boldsymbol{F} \cdot \overline{\boldsymbol{G}}_{\alpha}$, the derivative $\partial \overline{\boldsymbol{n}} / \partial \overline{\boldsymbol{F}} \equiv \partial \overline{\boldsymbol{n}} / \partial \boldsymbol{F}$. Considering an infinitesimal volume element and the definition of Jacobian, we have

$$
\mathrm{d} v=J \mathrm{~d} V \quad \Rightarrow \quad \mathrm{d} \boldsymbol{a} \cdot \mathrm{d} \boldsymbol{x}=J \mathrm{~d} \boldsymbol{A} \cdot \mathrm{d} \boldsymbol{X},
$$

in which $\mathrm{d} \boldsymbol{a}$ and $\mathrm{d} \boldsymbol{A}$ denote the interface area elements and $\mathrm{d} \boldsymbol{x}=\boldsymbol{F} \cdot \mathrm{d} \boldsymbol{X}$ and therefore

$$
\begin{gathered}
\mathrm{d} \boldsymbol{a} \cdot \boldsymbol{F} \cdot \mathrm{d} \boldsymbol{X}-J \mathrm{~d} \boldsymbol{A} \cdot \mathrm{d} \boldsymbol{X}=0 \Rightarrow[\mathrm{d} \boldsymbol{a} \cdot \boldsymbol{F}-J \mathrm{~d} \boldsymbol{A}] \cdot \mathrm{d} \boldsymbol{X}=0 \\
\Rightarrow \mathrm{d} \boldsymbol{a} \cdot \boldsymbol{F}=J \mathrm{~d} \boldsymbol{A} \Rightarrow \mathrm{d} \boldsymbol{a}=J \mathrm{~d} \boldsymbol{A} \cdot \boldsymbol{F}^{-1},
\end{gathered}
$$

being the celebrated Nanson formula $\mathrm{d} \boldsymbol{a}=J \boldsymbol{F}^{-\mathrm{t}} \cdot \mathrm{d} \boldsymbol{A}$ or $\mathrm{d} \boldsymbol{a}=\operatorname{Cof} \boldsymbol{F} \cdot \mathrm{d} \boldsymbol{A}$ on the interface. The Nanson formula can be interpreted as a linear normal map, as opposed to the linear tangent map $\boldsymbol{F}$, noting that $\mathrm{d} \boldsymbol{A}=\mathrm{d} A \overline{\boldsymbol{N}}$ and $\mathrm{d} \boldsymbol{a}=\mathrm{d} a \overline{\boldsymbol{n}}$ 
are the material and spatial area elements on the interface, respectively. Finally, we have

$$
\mathrm{d} a \overline{\boldsymbol{n}}=J \boldsymbol{F}^{-\mathrm{t}} \cdot[\mathrm{d} A \overline{\boldsymbol{N}}] \quad \Rightarrow \quad \overline{\boldsymbol{n}}=J \boldsymbol{F}^{-\mathrm{t}} \cdot \overline{\boldsymbol{N}} \frac{\mathrm{d} A}{\mathrm{~d} a}=\frac{J}{\bar{J}} \boldsymbol{F}^{-\mathrm{t}} \cdot \overline{\boldsymbol{N}} \quad \text { or } \quad \overline{\boldsymbol{n}}=\frac{\boldsymbol{F}^{-\mathrm{t}} \cdot \overline{\boldsymbol{N}}}{\left|\boldsymbol{F}^{-\mathrm{t}} \cdot \overline{\boldsymbol{N}}\right|},
$$

recalling that $\bar{J}=\mathrm{d} a / \mathrm{d} A$ denotes the interface Jacobian. ${ }^{8}$ We proceed using Eq. (B.29) 3 and the identities

$$
\frac{\partial}{\partial \boldsymbol{F}}\left(\frac{\boldsymbol{u}}{|\boldsymbol{u}|}\right)=\frac{1}{|\boldsymbol{u}|}\left[\boldsymbol{i}-\frac{\boldsymbol{u}}{|\boldsymbol{u}|} \otimes \frac{\boldsymbol{u}}{|\boldsymbol{u}|}\right] \cdot \frac{\partial \boldsymbol{u}}{\partial \boldsymbol{F}}, \quad \frac{\partial|\boldsymbol{u}|}{\partial \boldsymbol{F}}=\frac{\boldsymbol{u}}{|\boldsymbol{u}|} \cdot \frac{\partial \boldsymbol{u}}{\partial \boldsymbol{F}} \quad \forall \boldsymbol{u}: \text { arbitrary vector }
$$

and therefore

$$
\begin{aligned}
\frac{\partial \overline{\boldsymbol{n}}}{\partial \boldsymbol{F}}=\frac{\partial}{\partial \boldsymbol{F}}\left(\frac{\boldsymbol{F}^{-\mathrm{t}} \cdot \overline{\boldsymbol{N}}}{\left|\boldsymbol{F}^{-\mathrm{t}} \cdot \overline{\boldsymbol{N}}\right|}\right) & =\frac{1}{\left|\boldsymbol{F}^{-\mathrm{t}} \cdot \overline{\boldsymbol{N}}\right|}[\boldsymbol{i}-\underbrace{\frac{\boldsymbol{F}^{-\mathrm{t}} \cdot \overline{\boldsymbol{N}}}{\left|\boldsymbol{F}^{-\mathrm{t}} \cdot \overline{\boldsymbol{N}}\right|}}_{\overline{\boldsymbol{n}}} \otimes \underbrace{\frac{\boldsymbol{F}^{-\mathrm{t}} \cdot \overline{\boldsymbol{N}}}{\left|\boldsymbol{F}^{-\mathrm{t}} \cdot \overline{\boldsymbol{N}}\right|}}_{\overline{\boldsymbol{n}}}] \cdot \frac{\partial \boldsymbol{F}^{-\mathrm{t}} \cdot \overline{\boldsymbol{N}}}{\partial \boldsymbol{F}} \\
& =\frac{1}{\left|\boldsymbol{F}^{-\mathrm{t}} \cdot \overline{\boldsymbol{N}}\right|}[\boldsymbol{i}-\overline{\boldsymbol{n}} \otimes \overline{\boldsymbol{n}}] \cdot \frac{\partial \boldsymbol{F}^{-\mathrm{t} \cdot \overline{\boldsymbol{N}}}}{\partial \boldsymbol{F}},
\end{aligned}
$$

using the definition of the interface identity in the spatial configuration $\overline{\boldsymbol{i}}=\boldsymbol{i}-\overline{\boldsymbol{n}} \otimes \overline{\boldsymbol{n}}$ together with the identities $\partial \boldsymbol{F}^{-\mathrm{t}} / \partial \boldsymbol{F}=-\boldsymbol{F}^{-\mathrm{t}} \underline{\otimes} \boldsymbol{F}^{-1}$ or $\partial \boldsymbol{F}^{-1} / \partial \boldsymbol{F}=-\boldsymbol{F}^{-1} \bar{\otimes} \boldsymbol{F}^{-\mathrm{t}}$,

$$
\begin{aligned}
& =-\frac{1}{\left|\boldsymbol{F}^{-\mathrm{t}} \cdot \overline{\boldsymbol{N}}\right|} \overline{\boldsymbol{i}} \cdot\left[\boldsymbol{F}^{-\mathrm{t}} \bar{\otimes}\left[\boldsymbol{F}^{-\mathrm{t}} \cdot \overline{\boldsymbol{N}}\right]\right] \\
& =-\overline{\boldsymbol{i}} \cdot[\boldsymbol{F}^{-\mathrm{t}} \bar{\otimes} \underbrace{\left.\frac{\boldsymbol{F}^{-\mathrm{t}} \cdot \overline{\boldsymbol{N}}}{\left|\boldsymbol{F}^{-\mathrm{t}} \cdot \overline{\boldsymbol{N}}\right|}\right]=-\overline{\boldsymbol{i}} \cdot\left[\boldsymbol{F}^{-\mathrm{t}} \bar{\otimes} \overline{\boldsymbol{n}}\right]=-\underbrace{\left[\overline{\left.\boldsymbol{i} \cdot \boldsymbol{F}^{-\mathrm{t}}\right]}\right.}_{\overline{\boldsymbol{F}}^{-\mathrm{t}}} \bar{\otimes} \overline{\boldsymbol{n}}=-\overline{\boldsymbol{F}}^{-\mathrm{t}} \bar{\otimes} \overline{\boldsymbol{n}}=-\overline{\boldsymbol{n}} \bar{\otimes} \overline{\boldsymbol{F}}^{-\mathrm{t}},}_{\bar{n}}
\end{aligned}
$$

in which the relation $\overline{\boldsymbol{F}}^{-\mathrm{t}}=\overline{\boldsymbol{i}} \cdot \boldsymbol{F}^{-\mathrm{t}}$ follows as the transpose of $\overline{\boldsymbol{F}}^{-1}=\boldsymbol{F}^{-1} \cdot \overline{\boldsymbol{i}}$ in analogy to $\overline{\boldsymbol{F}}=\boldsymbol{F} \cdot \overline{\boldsymbol{I}}$. Therefore

$$
\frac{\partial \overline{\boldsymbol{n}}}{\partial \overline{\boldsymbol{F}}} \equiv \frac{\partial \overline{\boldsymbol{n}}}{\partial \boldsymbol{F}}=-\overline{\boldsymbol{n}} \bar{\otimes} \overline{\boldsymbol{F}}^{-\mathrm{t}}
$$

It is enlightening to further analyze the $\delta \overline{\boldsymbol{n}}=\partial \overline{\boldsymbol{n}} / \partial \overline{\boldsymbol{F}}: \delta \overline{\boldsymbol{F}}$. To do so, we note the structure of the interface deformation gradient and other relevant quantities

$$
\overline{\boldsymbol{F}}=\overline{\boldsymbol{g}}_{\alpha} \otimes \overline{\boldsymbol{G}}^{\alpha}, \quad \overline{\boldsymbol{F}}^{-1}=\overline{\boldsymbol{G}}_{\alpha} \otimes \overline{\boldsymbol{g}}^{\alpha}, \quad \overline{\boldsymbol{F}}^{-\mathrm{t}}=\overline{\boldsymbol{g}}^{\alpha} \otimes \overline{\boldsymbol{G}}_{\alpha}, \quad \delta \overline{\boldsymbol{F}}=\delta \overline{\boldsymbol{g}}_{\alpha} \otimes \overline{\boldsymbol{G}}^{\alpha},
$$

and therefore

$$
\delta \overline{\boldsymbol{n}}=\frac{\partial \overline{\boldsymbol{n}}}{\partial \overline{\boldsymbol{F}}}: \delta \overline{\boldsymbol{F}}=-\overline{\boldsymbol{n}} \bar{\otimes} \overline{\boldsymbol{F}}^{-\mathrm{t}}: \delta \overline{\boldsymbol{F}}=-\overline{\boldsymbol{n}} \cdot \delta \overline{\boldsymbol{F}} \cdot \overline{\boldsymbol{F}}^{-1}=-\overline{\boldsymbol{n}} \cdot \delta \overline{\boldsymbol{g}}_{\alpha} \otimes \overline{\boldsymbol{G}}^{\alpha} \cdot \overline{\boldsymbol{G}}_{\beta} \otimes \overline{\boldsymbol{g}}^{\beta}=-\left[\overline{\boldsymbol{n}} \cdot \delta \overline{\boldsymbol{g}}_{\alpha}\right] \overline{\boldsymbol{g}}^{\alpha},
$$

in which the coefficient $\overline{\boldsymbol{n}} \cdot \delta \overline{\boldsymbol{g}}_{\alpha}$ is non-zero since the variation of the basis vector $\delta \overline{\boldsymbol{g}}_{\alpha}$ is not necessarily tangential to the interface. Furthermore, the vector $\delta \overline{\boldsymbol{n}}$ is parallel to a linear combination of the contravariant basis in the spatial configuration $\overline{\boldsymbol{g}}^{\alpha}$. That is, $\delta \overline{\boldsymbol{n}}$ is indeed tangential to the interface in the spatial configuration and orthogonal to the interface normal itself or equivalently $\delta \overline{\boldsymbol{n}} \cdot \overline{\boldsymbol{n}}=\overline{\boldsymbol{n}} \cdot \delta \overline{\boldsymbol{n}}=0$. This result is not surprising as we expect it due to the property $\overline{\boldsymbol{n}} \cdot \overline{\boldsymbol{n}}=1$, nevertheless, to observe it through the derivation is reassuring.

\footnotetext{
${ }^{8}$ Using the relation $\overline{\boldsymbol{n}} \cdot \overline{\boldsymbol{n}}=1$, the interface Jacobian $\bar{J}$ can be expressed purely in terms of bulk quantities via the relation

$$
\bar{J}=J \sqrt{\left[\boldsymbol{F}^{-1} \cdot \boldsymbol{F}^{-\mathrm{t}}\right]:[\overline{\boldsymbol{N}} \otimes \overline{\boldsymbol{N}}]}=J \sqrt{B:[\overline{\boldsymbol{N}} \otimes \overline{\boldsymbol{N}}]}
$$

with $\boldsymbol{B}=\boldsymbol{F}^{-1} \cdot \boldsymbol{F}^{-t}$ being the Piola deformation tensor or the inverse of the right Cauchy-Green deformation tensor $\boldsymbol{B}=\boldsymbol{C}^{-1}$.
} 


\section{Appendix C. Proof of key identities regarding homogenization}

\section{C.1. Proof of the lemma (25)}

In order to prove the lemma

$$
\langle\boldsymbol{F}\rangle_{\mathcal{B}_{0}}+\langle\llbracket \varphi \rrbracket \otimes \bar{N}\rangle_{\mathcal{I}_{0}}=\frac{1}{\mathscr{V}_{0}} \int_{\partial \mathcal{B}_{0}} \boldsymbol{\varphi} \otimes \widehat{N} \mathrm{~d} A,
$$

we start from the right-hand side and add/subtract the second integral as

$$
\frac{1}{\mathscr{V}_{0}} \int_{\partial \mathcal{B}_{0}} \boldsymbol{\varphi} \otimes \widehat{N} \mathrm{~d} A=\frac{1}{\mathscr{V}_{0}} \int_{\partial \mathcal{B}_{0}} \boldsymbol{\varphi} \otimes \widehat{N} \mathrm{~d} A+\frac{1}{\mathscr{V}_{0}} \int_{\mathcal{I}_{0}^{-}} \boldsymbol{\varphi} \otimes \widehat{N} \mathrm{~d} A-\frac{1}{\mathscr{V}_{0}} \int_{\mathcal{I}_{0}^{-}} \boldsymbol{\varphi} \otimes \widehat{N} \mathrm{~d} A,
$$

employing $\widehat{N}=\bar{N}$ on $\mathcal{I}_{0}^{-}$and $\varphi^{-}=\varphi^{+}-\llbracket \varphi \rrbracket$ on the last integral

$$
\begin{aligned}
\frac{1}{\mathscr{V}_{0}} \int_{\partial \mathcal{B}_{0}} \varphi \otimes \widehat{N} \mathrm{~d} A= & \frac{1}{\mathscr{V}_{0}} \int_{\partial \mathcal{B}_{0}} \varphi \otimes \widehat{N} \mathrm{~d} A+\frac{1}{\mathscr{V}_{0}} \int_{\mathcal{I}_{0}^{-}} \varphi \otimes \widehat{N} \mathrm{~d} A \\
& -\frac{1}{\mathscr{V}_{0}} \int_{\mathcal{I}_{0}^{-}}\left[\varphi^{+}-\llbracket \varphi \rrbracket\right] \otimes \bar{N} \mathrm{~d} A,
\end{aligned}
$$

writing the first two integrals as the union of their domains via $\partial \mathcal{B}_{0}^{-}=\partial \mathcal{B}_{0} \cup \mathcal{I}_{0}^{-}$, splitting the last integral using $\left.\widehat{\boldsymbol{N}}\right|_{\mathcal{I}_{0}^{+}}=-\overline{\boldsymbol{N}}$ and that $\mathcal{I}_{0}=\mathcal{I}_{0}^{+}=\mathcal{I}_{0}^{-}$geometrically,

$$
\frac{1}{\mathscr{V}_{0}} \int_{\partial \mathcal{B}_{0}} \varphi \otimes \widehat{N} \mathrm{~d} A=\frac{1}{\mathscr{V}_{0}} \int_{\partial \mathcal{B}_{0}^{-}} \varphi \otimes \widehat{N} \mathrm{~d} A+\frac{1}{\mathscr{V}_{0}} \int_{\mathcal{I}_{0}} \llbracket \varphi \rrbracket \otimes \bar{N} \mathrm{~d} A+\frac{1}{\mathscr{V}_{0}} \int_{\mathcal{I}_{0}^{+}} \varphi \otimes \widehat{N} \mathrm{~d} A,
$$

replacing $\mathcal{I}_{0}^{+}$with $\partial \mathcal{B}_{0}^{+}$,

$$
\frac{1}{\mathscr{V}_{0}} \int_{\partial \mathcal{B}_{0}} \boldsymbol{\varphi} \otimes \widehat{N} \mathrm{~d} A=\frac{1}{\mathscr{V}_{0}} \int_{\partial \mathcal{B}_{0}^{-}} \boldsymbol{\varphi} \otimes \widehat{\boldsymbol{N}} \mathrm{d} A+\frac{1}{\mathscr{V}_{0}} \int_{\mathcal{I}_{0}} \llbracket \varphi \rrbracket \otimes \overline{\boldsymbol{N}} \mathrm{d} A+\frac{1}{\mathscr{V}_{0}} \int_{\partial \mathcal{B}_{0}^{+}} \boldsymbol{\varphi} \otimes \widehat{\boldsymbol{N}} \mathrm{d} A
$$

employing the gradient theorem on the first and the last term

$$
\begin{aligned}
\frac{1}{\mathscr{V}_{0}} \int_{\partial \mathcal{B}_{0}} \boldsymbol{\varphi} \otimes \widehat{\boldsymbol{N}} \mathrm{d} A & =\frac{1}{\mathscr{V}_{0}} \int_{\mathcal{B}_{0}^{-}} \operatorname{Grad} \boldsymbol{i} \mathrm{d} V+\frac{1}{\mathscr{V}_{0}} \int_{\mathcal{I}_{0}} \llbracket \varphi \rrbracket \otimes \overline{\boldsymbol{N}} \mathrm{d} A+\frac{1}{\mathscr{V}_{0}} \int_{\mathcal{B}_{0}^{+}} \operatorname{Grad} \boldsymbol{\varphi} \mathrm{d} V \\
& =\frac{1}{\mathscr{V}_{0}} \int_{\mathcal{B}_{0}^{-}} \boldsymbol{F} \mathrm{d} V+\frac{1}{\mathscr{V}_{0}} \int_{\mathcal{I}_{0}} \llbracket \boldsymbol{\varphi} \rrbracket \otimes \overline{\boldsymbol{N}} \mathrm{d} A+\frac{1}{\mathscr{V}_{0}} \int_{\mathcal{B}_{0}^{+}} \boldsymbol{F} \mathrm{d} V \\
& =\langle\boldsymbol{F}\rangle_{\mathcal{B}_{0}^{-}}+\frac{1}{\mathscr{V}_{0}} \int_{\mathcal{I}_{0}} \llbracket \boldsymbol{\varphi} \rrbracket \otimes \overline{\boldsymbol{N}} \mathrm{d} A+\langle\boldsymbol{F}\rangle_{\mathcal{B}_{0}^{+}} \\
& =\langle\boldsymbol{F}\rangle_{\mathcal{B}_{0}}+\langle\llbracket \varphi \rrbracket \otimes \overline{\boldsymbol{N}}\rangle_{\mathcal{I}_{0}} .
\end{aligned}
$$

\section{C.2. Proof of the lemma (28)}

In order to prove the lemma

$$
\langle\boldsymbol{P}\rangle_{\mathcal{B}_{0}}+\langle\overline{\boldsymbol{P}}\rangle_{\mathcal{I}_{0}}=\frac{1}{\mathscr{V}_{0}} \int_{\partial \mathcal{B}_{0}} \widehat{\boldsymbol{b}}_{0} \otimes \boldsymbol{X} \mathrm{d} A,
$$

we start from the right-hand side by replacing the traction $\widehat{\boldsymbol{b}}_{0}$ with its representation in terms of Piola stress as $\widehat{\boldsymbol{b}}_{0}=\boldsymbol{P} \cdot \widehat{\boldsymbol{N}}$ and add/subtract the second integral as

$$
\frac{1}{\mathscr{V}_{0}} \int_{\partial \mathcal{B}_{0}} \widehat{\boldsymbol{b}}_{0} \otimes \boldsymbol{X} \mathrm{d} A=\frac{1}{\mathscr{V}_{0}} \int_{\partial \mathcal{B}_{0}}[\boldsymbol{P} \cdot \widehat{\boldsymbol{N}}] \otimes \boldsymbol{X} \mathrm{d} A+\frac{1}{\mathscr{V}_{0}} \int_{\mathcal{I}_{0}^{-}}[\boldsymbol{P} \cdot \widehat{\boldsymbol{N}}] \otimes \overline{\boldsymbol{X}} \mathrm{d} A-\frac{1}{\mathscr{V}_{0}} \int_{\mathcal{I}_{0}^{-}}[\boldsymbol{P} \cdot \widehat{\boldsymbol{N}}] \otimes \overline{\boldsymbol{X}} \mathrm{d} A,
$$


employing the balance of linear momentum on the interface (11) with $\widehat{N}=\bar{N}$ on $\mathcal{I}_{0}^{-}$, we obtain

$$
\begin{aligned}
\frac{1}{\mathscr{V}_{0}} \int_{\partial \mathcal{B}_{0}} \widehat{\boldsymbol{b}}_{0} \otimes \boldsymbol{X} \mathrm{d} A= & \frac{1}{\mathscr{V}_{0}} \int_{\partial \mathcal{B}_{0}}[\boldsymbol{P} \cdot \widehat{\boldsymbol{N}}] \otimes \boldsymbol{X} \mathrm{d} A+\frac{1}{\mathscr{V}_{0}} \int_{\mathcal{I}_{0}^{-}}[\boldsymbol{P} \cdot \widehat{\boldsymbol{N}}] \otimes \overline{\boldsymbol{X}} \mathrm{d} A \\
& -\frac{1}{\mathscr{V}_{0}} \int_{\mathcal{I}_{0-}}\left[\overline{\operatorname{Div}} \overline{\boldsymbol{P}}+\boldsymbol{P}^{+} \cdot \overline{\boldsymbol{N}}\right] \otimes \overline{\boldsymbol{X}} \mathrm{d} A,
\end{aligned}
$$

writing the first two integrals as the union of their domains via $\partial \mathcal{B}_{0}^{-}=\partial \mathcal{B}_{0} \cup \mathcal{I}_{0}^{-}$, utilizing the non-standard product $\bar{\otimes}$ between a vector and a second-order tensor as $[\boldsymbol{P} \cdot \widehat{N}] \otimes X=[\boldsymbol{X} \bar{\otimes} \boldsymbol{P}] \cdot \widehat{\boldsymbol{N}}$, splitting the last integral applying $\left.\widehat{\boldsymbol{N}}\right|_{\mathcal{I}_{0}^{+}}=-\overline{\boldsymbol{N}}$ and that $\mathcal{I}_{0}=\mathcal{I}_{0}^{+}=\mathcal{I}_{0}^{-}$holds geometrically,

$$
\frac{1}{\mathscr{V}_{0}} \int_{\partial \mathcal{B}_{0}} \widehat{\boldsymbol{b}}_{0} \otimes \boldsymbol{X} \mathrm{d} A=\frac{1}{\mathscr{V}_{0}} \int_{\partial \mathcal{B}_{0}^{-}}[\boldsymbol{X} \bar{\otimes} \boldsymbol{P}] \cdot \widehat{\boldsymbol{N}} \mathrm{d} A-\frac{1}{\mathscr{V}_{0}} \int_{\mathcal{I}_{0}} \overline{\operatorname{Div}} \overline{\boldsymbol{P}} \otimes \overline{\boldsymbol{X}} \mathrm{d} A+\frac{1}{\mathscr{V}_{0}} \int_{\mathcal{I}_{0}+}\left[\boldsymbol{P}^{+} \cdot \widehat{\boldsymbol{N}}\right] \otimes \overline{\boldsymbol{X}} \mathrm{d} A,
$$

using the identity $\overline{\operatorname{Div}} \overline{\boldsymbol{P}} \otimes \overline{\boldsymbol{X}}=\overline{\operatorname{Div}}(\overline{\boldsymbol{X}} \bar{\otimes} \overline{\boldsymbol{P}})-\overline{\boldsymbol{P}} \cdot \overline{\operatorname{Grad}} \overline{\boldsymbol{X}}=\overline{\operatorname{Div}}(\overline{\boldsymbol{X}} \bar{\otimes} \overline{\boldsymbol{P}})-\overline{\boldsymbol{P}}$ on the second term and replacing $\mathcal{I}_{0}^{+}$ with $\partial \mathcal{B}_{0}^{+}$with $[\boldsymbol{P} \cdot \widehat{N}] \otimes X=[\boldsymbol{X} \bar{\otimes}] \cdot \widehat{\boldsymbol{N}}$,

$$
\frac{1}{\mathscr{V}_{0}} \int_{\partial \mathcal{B}_{0}} \widehat{\boldsymbol{b}}_{0} \otimes \boldsymbol{X} \mathrm{d} A=\frac{1}{\mathscr{V}_{0}} \int_{\partial \mathcal{B}_{0}^{-}}[\boldsymbol{X} \bar{\otimes} \boldsymbol{P}] \cdot \widehat{\boldsymbol{N}} \mathrm{d} A-\frac{1}{\mathscr{V}_{0}} \int_{\mathcal{I}_{0}} \overline{\operatorname{Div}}(\overline{\boldsymbol{X}} \bar{\otimes} \overline{\boldsymbol{P}})-\overline{\boldsymbol{P}} \mathrm{d} A+\frac{1}{\mathscr{V}_{0}} \int_{\partial \mathcal{B}_{0}^{+}}[\boldsymbol{X} \bar{\otimes} \boldsymbol{P}] \cdot \widehat{\boldsymbol{N}} \mathrm{d} A,
$$

applying the divergence theorem on the first and the last terms and splitting the second term,

$$
\begin{aligned}
\frac{1}{\mathscr{V}_{0}} \int_{\partial \mathcal{B}_{0}} \widehat{\boldsymbol{b}}_{0} \otimes \boldsymbol{X} \mathrm{d} A= & \frac{1}{\mathscr{V}_{0}} \int_{\mathcal{B}_{0}^{-}} \operatorname{Div}(\boldsymbol{X} \bar{\otimes} \boldsymbol{P}) \mathrm{d} V-\frac{1}{\mathscr{V}_{0}} \int_{\mathcal{I}_{0}} \overline{\operatorname{Div}}(\overline{\boldsymbol{X}} \bar{\otimes} \overline{\boldsymbol{P}}) \mathrm{d} A+\frac{1}{\mathscr{V}_{0}} \int_{\mathcal{I}_{0}} \overline{\boldsymbol{P}} \mathrm{d} A \\
& +\frac{1}{\mathscr{V}_{0}} \int_{\mathcal{B}_{0}^{+}} \operatorname{Div}(\boldsymbol{X} \bar{\otimes} \boldsymbol{P}) \mathrm{d} V,
\end{aligned}
$$

with the identity $\operatorname{Div}(\boldsymbol{X} \bar{\otimes} \boldsymbol{P})=\operatorname{Div} \boldsymbol{P} \otimes \boldsymbol{X}+\boldsymbol{P} \cdot \operatorname{Grad} \boldsymbol{X}=\boldsymbol{P}$ due to the balance of linear momentum in the bulk, on the first and the last terms and using the interface divergence theorem (B.3) on the second term,

$$
\begin{aligned}
\frac{1}{\mathscr{V}_{0}} \int_{\partial \mathcal{B}_{0}} \widehat{\boldsymbol{b}}_{0} \otimes \boldsymbol{X} \mathrm{d} A= & \frac{1}{\mathscr{V}_{0}} \int_{\mathcal{B}_{0}^{-}} \boldsymbol{P} \mathrm{d} V-\frac{1}{\mathscr{V}_{0}} \int_{\partial \mathcal{I}_{0}}[\overline{\boldsymbol{X}} \bar{\otimes} \overline{\boldsymbol{P}}] \cdot \tilde{\boldsymbol{N}} \mathrm{d} L \\
& +\frac{1}{\mathscr{V}_{0}} \int_{\mathcal{I}_{0}} \bar{K}[\overline{\boldsymbol{X}} \bar{\otimes} \overline{\boldsymbol{P}}] \cdot \overline{\boldsymbol{N}} \mathrm{d} A+\frac{1}{\mathscr{V}_{0}} \int_{\mathcal{I}_{0}} \overline{\boldsymbol{P}} \mathrm{d} A+\frac{1}{\mathscr{V}_{0}} \int_{\mathcal{B}_{0}^{+}} \boldsymbol{P} \mathrm{d} V,
\end{aligned}
$$

thereby the second integral vanishes due to $\partial \mathcal{I}_{0}=\emptyset$ and the third integral vanishes due to the superficiality of the interface Piola stress $\overline{\boldsymbol{P}} \cdot \overline{\boldsymbol{N}}=\mathbf{0}$ and finally we arrive at

$$
\begin{aligned}
\frac{1}{\mathscr{V}_{0}} \int_{\partial \mathcal{B}_{0}} \widehat{\boldsymbol{b}}_{0} \otimes \boldsymbol{X} \mathrm{d} A & =\frac{1}{\mathscr{V}_{0}} \int_{\mathcal{B}_{0}^{-}} \boldsymbol{P} \mathrm{d} V+\frac{1}{\mathscr{V}_{0}} \int_{\mathcal{I}_{0}} \overline{\boldsymbol{P}} \mathrm{d} A+\frac{1}{\mathscr{V}_{0}} \int_{\mathcal{B}_{0}^{+}} \boldsymbol{P} \mathrm{d} V \\
& =\langle\boldsymbol{P}\rangle_{\mathcal{B}_{0}^{-}}+\langle\overline{\boldsymbol{P}}\rangle_{\mathcal{I}_{0}}+\langle\boldsymbol{P}\rangle_{\mathcal{B}_{0}^{+}} \\
& =\langle\boldsymbol{P}\rangle_{\mathcal{B}_{0}}+\langle\overline{\boldsymbol{P}}\rangle_{\mathcal{I}_{0}} .
\end{aligned}
$$

\section{C.3. Proof of the lemma (32)}

In order to prove the lemma

$$
\frac{1}{\mathscr{V}_{0}} \int_{\partial \mathcal{B}_{0}} \widehat{\boldsymbol{b}}_{0} \cdot \delta \boldsymbol{\varphi} \mathrm{d} A=\langle\boldsymbol{P}: \delta \boldsymbol{F}\rangle_{\mathcal{B}_{0}}+\langle\overline{\boldsymbol{P}}: \delta \overline{\boldsymbol{F}}\rangle_{\mathcal{I}_{0}}+\langle\{\{\boldsymbol{t}\}] \cdot \llbracket \delta \boldsymbol{\varphi} \rrbracket\rangle_{\mathcal{I}_{0}},
$$


we start from the left-hand side as

$$
\begin{aligned}
\frac{1}{\mathscr{V}_{0}} \int_{\partial \mathcal{B}_{0}} \widehat{\boldsymbol{b}}_{0} \cdot \delta \boldsymbol{\varphi} \mathrm{d} A= & \frac{1}{\mathscr{V}_{0}} \int_{\partial \mathcal{B}_{0}}[\delta \boldsymbol{\varphi} \cdot \boldsymbol{P}] \cdot \widehat{\boldsymbol{N}} \mathrm{d} A+\frac{1}{\mathscr{V}_{0}} \int_{\mathcal{I}_{0}^{-}}[\delta \boldsymbol{\varphi} \cdot \boldsymbol{P}] \cdot \widehat{\boldsymbol{N}} \mathrm{d} A-\frac{1}{\mathscr{V}_{0}} \int_{\mathcal{I}_{0}^{-}}[\delta \boldsymbol{\varphi} \cdot \boldsymbol{P}] \cdot \widehat{\boldsymbol{N}} \mathrm{d} A \\
= & \frac{1}{\mathscr{V}_{0}} \int_{\partial \mathcal{B}_{0}^{-}}[\delta \boldsymbol{\varphi} \cdot \boldsymbol{P}] \cdot \widehat{\boldsymbol{N}} \mathrm{d} A-\frac{1}{\mathscr{V}_{0}} \int_{\mathcal{I}_{0}^{-}}[\delta \boldsymbol{\varphi} \cdot \boldsymbol{P}] \cdot \overline{\boldsymbol{N}} \mathrm{d} A \pm \frac{1}{\mathscr{V}_{0}} \int_{\mathcal{I}_{0}^{+}}[\delta \boldsymbol{\varphi} \cdot \boldsymbol{P}] \cdot \overline{\boldsymbol{N}} \mathrm{d} A \\
= & \frac{1}{\mathscr{V}_{0}} \int_{\partial \mathcal{B}_{0}^{-}}[\delta \boldsymbol{\varphi} \cdot \boldsymbol{P}] \cdot \widehat{\boldsymbol{N}} \mathrm{d} A+\frac{1}{\mathscr{V}_{0}} \int_{\mathcal{I}_{0}} \llbracket \delta \boldsymbol{\varphi} \cdot \boldsymbol{P} \rrbracket \cdot \overline{\boldsymbol{N}} \mathrm{d} A-\frac{1}{\mathscr{V}_{0}} \int_{\mathcal{I}_{0}^{+}}[\delta \boldsymbol{\varphi} \cdot \boldsymbol{P}] \cdot \overline{\boldsymbol{N}} \mathrm{d} A \\
= & \frac{1}{\mathscr{V}_{0}} \int_{\partial \mathcal{B}_{0}^{-}}[\delta \boldsymbol{\varphi} \cdot \boldsymbol{P}] \cdot \widehat{\boldsymbol{N}} \mathrm{d} A+\frac{1}{\mathscr{V}_{0}} \int_{\mathcal{I}_{0}} \llbracket \delta \boldsymbol{\varphi} \cdot \boldsymbol{P} \rrbracket \cdot \overline{\boldsymbol{N}} \mathrm{d} A+\frac{1}{\mathscr{V}_{0}} \int_{\partial \mathcal{B}_{0}^{+}}[\delta \boldsymbol{\varphi} \cdot \boldsymbol{P}] \cdot \widehat{\boldsymbol{N}} \mathrm{d} A \\
= & \frac{1}{\mathscr{V}_{0}} \int_{\mathcal{B}_{0}^{-}} \operatorname{Div}(\delta \boldsymbol{\varphi} \cdot \boldsymbol{P}) \mathrm{d} V+\frac{1}{\mathscr{V}_{0}} \int_{\mathcal{I}_{0}}[\llbracket \delta \boldsymbol{\varphi} \rrbracket \cdot\{\boldsymbol{P}\}]+\llbracket \delta \boldsymbol{\varphi} \rrbracket \cdot\{\{\boldsymbol{P}\}] \cdot \overline{\boldsymbol{N}} \mathrm{d} A \\
& +\frac{1}{\mathscr{V}_{0}} \int_{\mathcal{B}_{0}^{+}} \operatorname{Div}(\delta \boldsymbol{\varphi} \cdot \boldsymbol{P}) \mathrm{d} V \\
= & \frac{1}{\mathscr{V}_{0}} \int_{\mathcal{B}_{0}^{-}} \boldsymbol{P}: \delta \boldsymbol{F} \mathrm{d} V+\frac{1}{\mathscr{V}_{0}} \int_{\mathcal{I}_{0}}\{[\boldsymbol{t}\}] \cdot \llbracket \delta \boldsymbol{\varphi} \rrbracket \mathrm{d} A+\frac{1}{\mathscr{V}_{0}} \int_{\mathcal{I}_{0}}\{\{\delta \boldsymbol{\varphi}\}] \cdot \llbracket \boldsymbol{t} \rrbracket \mathrm{d} A \\
& +\frac{1}{\mathscr{V}_{0}} \int_{\mathcal{B}_{0}^{+}} \boldsymbol{P}: \delta \boldsymbol{F} \mathrm{d} V
\end{aligned}
$$

using the balance of linear momentum on the interface and the assumption $\bar{\varphi}=\{\{\varphi\}$,

$$
\begin{aligned}
\frac{1}{\mathscr{V}_{0}} \int_{\partial \mathcal{B}_{0}} \widehat{\boldsymbol{b}}_{0} \cdot \delta \boldsymbol{\varphi} \mathrm{d} A= & \langle\boldsymbol{P}: \delta \boldsymbol{F}\rangle_{\mathcal{B}_{0}^{-}}+\langle\{\{\boldsymbol{t}\}] \cdot \llbracket \delta \boldsymbol{\varphi} \rrbracket\rangle_{\mathcal{I}_{0}}-\frac{1}{\mathscr{V}_{0}} \int_{\mathcal{I}_{0}} \delta \overline{\boldsymbol{\varphi}} \cdot \overline{\operatorname{Div}} \overline{\boldsymbol{P}} \mathrm{d} A+\langle\boldsymbol{P}: \delta \boldsymbol{F}\rangle_{\mathcal{B}_{0}^{+}} \\
= & \langle\boldsymbol{P}: \delta \boldsymbol{F}\rangle_{\mathcal{B}_{0}}+\langle\{\{\boldsymbol{t}\}] \cdot \llbracket \delta \boldsymbol{\varphi} \rrbracket\rangle_{\mathcal{I}_{0}}-\frac{1}{\mathscr{V}_{0}} \int_{\mathcal{I}_{0}} \overline{\operatorname{Div}}(\delta \overline{\boldsymbol{\varphi}} \cdot \overline{\boldsymbol{P}})-\overline{\boldsymbol{P}}: \delta \overline{\boldsymbol{F}} \mathrm{d} A \\
= & \langle\boldsymbol{P}: \delta \boldsymbol{F}\rangle_{\mathcal{B}_{0}}+\langle\{[\boldsymbol{t}\}] \cdot \llbracket \delta \boldsymbol{\varphi} \rrbracket\rangle_{\mathcal{I}_{0}}+\langle\overline{\boldsymbol{P}}: \delta \overline{\boldsymbol{F}}\rangle_{\mathcal{I}_{0}}-\frac{1}{\mathscr{V}_{0}} \int_{\partial \mathcal{I}_{0}}[\delta \overline{\boldsymbol{\varphi}} \cdot \overline{\boldsymbol{P}}] \cdot \tilde{\boldsymbol{N}} \mathrm{d} L \\
& +\frac{1}{\mathscr{V}_{0}} \int_{\mathcal{I}_{0}} K \delta \overline{\boldsymbol{\varphi}} \cdot \overline{\boldsymbol{P}} \cdot \overline{\boldsymbol{N}} \mathrm{d} A,
\end{aligned}
$$

thereby the last two integrals vanish due to $\partial \mathcal{I}_{0}=\emptyset$ and the superficiality of the interface Piola stress $\overline{\boldsymbol{P}} \cdot \overline{\boldsymbol{N}}=\mathbf{0}$ and finally we arrive at

$$
\frac{1}{\mathscr{V}_{0}} \int_{\partial \mathcal{B}_{0}} \widehat{\boldsymbol{b}}_{0} \cdot \delta \boldsymbol{\varphi} \mathrm{d} A=\langle\boldsymbol{P}: \delta \boldsymbol{F}\rangle_{\mathcal{B}_{0}}+\langle\{\{\boldsymbol{t}\}] \cdot \llbracket \delta \boldsymbol{\varphi} \rrbracket\rangle_{\mathcal{I}_{0}}+\langle\overline{\boldsymbol{P}}: \delta \overline{\boldsymbol{F}}\rangle_{\mathcal{I}_{0}}
$$

\section{C.4. Proof of the extended Hill identity (33)}

In order to prove the Hill identity

$$
\frac{1}{\mathscr{V}_{0}} \int_{\partial \mathcal{B}_{0}} \widehat{\boldsymbol{b}}_{0} \cdot \delta \boldsymbol{\varphi} \mathrm{d} A-{ }^{\mathrm{M}} \boldsymbol{P}: \delta^{\mathrm{M}} \boldsymbol{F}=\frac{1}{\mathscr{V}_{0}} \int_{\partial \mathcal{B}_{0}}\left[\delta \boldsymbol{\varphi}-\delta^{\mathrm{M}} \boldsymbol{F} \cdot \boldsymbol{X}\right] \cdot\left[\widehat{\boldsymbol{b}}_{0}-{ }^{\mathrm{M}} \boldsymbol{P} \cdot \widehat{\boldsymbol{N}}\right] \mathrm{d} A,
$$

by expanding the right-hand side and manipulating it until we remain with the left-hand side as

$$
\begin{aligned}
\frac{1}{\mathscr{V}_{0}} & \int_{\partial \mathcal{B}_{0}}\left[\delta \boldsymbol{\varphi}-\delta^{\mathrm{M}} \boldsymbol{F} \cdot \boldsymbol{X}\right] \cdot\left[\widehat{\boldsymbol{b}}_{0}-{ }^{\mathrm{M}} \boldsymbol{P} \cdot \widehat{\boldsymbol{N}}\right] \mathrm{d} A \\
& =\frac{1}{\mathscr{V}_{0}} \int_{\partial \mathcal{B}_{0}} \delta \boldsymbol{\varphi} \cdot \widehat{\boldsymbol{b}}_{0}-\delta \boldsymbol{\varphi} \cdot{ }^{\mathrm{M}} \boldsymbol{P} \cdot \widehat{\boldsymbol{N}}-\delta^{\mathrm{M}} \boldsymbol{F} \cdot \boldsymbol{X} \cdot \widehat{\boldsymbol{b}}_{0}+\left[\delta^{\mathrm{M}} \boldsymbol{F} \cdot \boldsymbol{X}\right] \cdot\left[{ }^{\mathrm{M}} \boldsymbol{P} \cdot \widehat{\boldsymbol{N}}\right] \mathrm{d} A \\
& =\frac{1}{\mathscr{V}_{0}} \int_{\partial \mathcal{B}_{0}} \delta \boldsymbol{\varphi} \cdot \widehat{\boldsymbol{b}}_{0}-\frac{1}{\mathscr{V}_{0}} \int_{\partial \mathcal{B}_{0}} \delta \boldsymbol{\varphi} \cdot{ }^{\mathrm{M}} \boldsymbol{P} \cdot \widehat{\boldsymbol{N}} \mathrm{d} A-\frac{1}{\mathscr{V}_{0}} \int_{\partial \mathcal{B}_{0}}\left[\delta^{\mathrm{M}} \boldsymbol{F} \cdot \boldsymbol{X}\right] \cdot \widehat{\boldsymbol{b}}_{0} \mathrm{~d} A
\end{aligned}
$$




$$
\begin{aligned}
& +\frac{1}{\mathscr{V}_{0}} \int_{\partial \mathcal{B}_{0}}\left[\delta^{\mathrm{M}} \boldsymbol{F} \cdot \boldsymbol{X}\right] \cdot\left[{ }^{\mathrm{M}} \boldsymbol{P} \cdot \widehat{\boldsymbol{N}}\right] \mathrm{d} A \\
= & \frac{1}{\mathscr{V}_{0}} \int_{\partial \mathcal{B}_{0}} \widehat{\boldsymbol{b}}_{0} \cdot \delta \boldsymbol{\varphi}-\frac{1}{\mathscr{V}_{0}}{ }^{\mathrm{M}} \boldsymbol{P}: \int_{\partial \mathcal{B}_{0}} \delta \boldsymbol{\varphi} \otimes \widehat{\boldsymbol{N}} \mathrm{d} A-\frac{1}{\mathscr{V}_{0}} \delta^{\mathrm{M}} \boldsymbol{F}: \int_{\partial \mathcal{B}_{0}} \widehat{\boldsymbol{b}}_{0} \otimes \boldsymbol{X} \mathrm{d} A \\
& +\frac{1}{\mathscr{V}_{0}} \delta^{\mathrm{M}} \boldsymbol{F} \cdot{ }^{\mathrm{M}} \boldsymbol{P}: \int_{\partial \mathcal{B}_{0}} \boldsymbol{X} \otimes \widehat{\boldsymbol{N}} \mathrm{d} A,
\end{aligned}
$$

using the definition of the macro deformation gradient (27) on the second term, the definition of the macro Piola stress (30) on the third term and the identity (24) on the last term,

$$
\frac{1}{\mathscr{V}_{0}} \int_{\partial \mathcal{B}_{0}}\left[\delta \boldsymbol{\varphi}-\delta^{\mathrm{M}} \boldsymbol{F} \cdot \boldsymbol{X}\right] \cdot\left[\widehat{\boldsymbol{b}}_{0}-{ }^{\mathrm{M}} \boldsymbol{P} \cdot \widehat{\boldsymbol{N}}\right] \mathrm{d} A=\frac{1}{\mathscr{V}_{0}} \int_{\partial \mathcal{B}_{0}} \widehat{\boldsymbol{b}}_{0} \cdot \delta \boldsymbol{\varphi}-\mathrm{M}_{\boldsymbol{P}}: \delta^{\mathrm{M}} \boldsymbol{F}-\delta^{\mathrm{M}} \boldsymbol{F}: \mathrm{M}^{\mathrm{P}}+\left[\delta^{\mathrm{M}} \boldsymbol{F} \cdot \mathrm{M}_{\boldsymbol{P}}\right]: \boldsymbol{I},
$$

since $\left[\delta^{\mathrm{M}} \boldsymbol{F} \cdot{ }^{\mathrm{M}} \boldsymbol{P}\right]: \boldsymbol{I}={ }^{\mathrm{M}} \boldsymbol{P}: \delta^{\mathrm{M}} \boldsymbol{F}$, the last two terms vanish together and finally

$$
\frac{1}{\mathscr{V}_{0}} \int_{\partial \mathcal{B}_{0}}\left[\delta \boldsymbol{\varphi}-\delta^{\mathrm{M}} \boldsymbol{F} \cdot \boldsymbol{X}\right] \cdot\left[\widehat{\boldsymbol{b}}_{0}-{ }^{\mathrm{M}} \boldsymbol{P} \cdot \widehat{\boldsymbol{N}}\right] \mathrm{d} A=\frac{1}{\mathscr{V}_{0}} \int_{\partial \mathcal{B}_{0}} \widehat{\boldsymbol{b}}_{0} \cdot \delta \boldsymbol{\varphi}-{ }^{\mathrm{M}} \boldsymbol{P}: \delta^{\mathrm{M}} \boldsymbol{F} .
$$

Alternatively, one could start by employing the divergence theorem and recast everything in terms of bulk and interface integrals instead of the boundary integral above. This alternative procedure renders directly the alternative format of the Hill lemma (33b) and is far more cumbersome. The aforementioned proof is not only more elegant but also more general since it is independent of the complexity of the micro-structure. The two formats of the Hill lemma (33a) and (33b) are readily connected via the lemma (32) proven in Appendix C.3.

\section{Appendix D. Detailed derivation of tangents}

In this section, detailed derivations to compute the tangents of the interface elements (48) from their corresponding residuals are given. The derivations of the tangent for the bulk (45) are standard and are omitted. The tangent stiffness of the interface is essentially composed of four similar parts. We limit the discussion to only one of them and via the relations below, it is straightforward to derive the remaining ones in a similar manner.

$$
\frac{\partial \bar{\varphi}}{\partial \varphi^{+}}=\frac{\partial\{\{\varphi\}}{\partial \varphi^{+}}=\frac{1}{2} \boldsymbol{I}, \quad \frac{\partial \bar{\varphi}}{\partial \varphi^{-}}=\frac{\partial\{\{\varphi\}}{\partial \varphi^{-}}=\frac{1}{2} \boldsymbol{I}, \quad \frac{\partial \llbracket \varphi \rrbracket}{\partial \varphi^{+}}=\boldsymbol{I}, \quad \frac{\partial \llbracket \varphi \rrbracket}{\partial \varphi^{-}}=-\boldsymbol{I},
$$

which follow from the definition of the jump and average operator on the interface. Thus the interface tangent expands as

$$
\begin{aligned}
& \overline{\mathbf{K}}_{++}^{I J}=\frac{\partial \overline{\mathbf{R}}_{+}^{I}}{\partial \varphi_{+}^{J}}=\frac{\partial}{\partial \varphi_{+}^{J}} \int_{\mathcal{I}_{0}} \frac{1}{2} \overline{\boldsymbol{P}} \cdot \overline{\operatorname{Grad}} \bar{N}^{I}+\{\{t\}\} \cdot \overline{\operatorname{Grad}} \bar{N}^{I} \mathrm{~d} A \\
& =\int_{\mathcal{I}_{0}} \frac{\partial}{\partial \varphi_{+}^{J}}\left(\frac{1}{2} \overline{\boldsymbol{P}} \cdot \overline{\operatorname{Grad}} \bar{N}^{I}+\{\{\boldsymbol{t}\}] \cdot \overline{\operatorname{Grad}} \bar{N}^{I}\right) \mathrm{d} A \\
& =\int_{\mathcal{I}_{0}} \overline{\operatorname{Grad}} \bar{N}^{I} \cdot \frac{\partial}{\partial \varphi_{+}^{J}}\left(\frac{1}{2} \overline{\boldsymbol{P}}+\{\{\boldsymbol{t}\}\}\right) \mathrm{d} A \\
& =\int_{\mathcal{I}_{0}} \overline{\operatorname{Grad}} \bar{N}^{I}=\left[\frac{\partial}{\partial \overline{\boldsymbol{F}}}\left(\frac{1}{2} \overline{\boldsymbol{P}}+\{\{\boldsymbol{t}\})\right): \frac{\partial \overline{\boldsymbol{F}}}{\partial \boldsymbol{\varphi}_{+}^{J}}\right]+\overline{\operatorname{Grad}} \bar{N}^{I}=\left[\frac{\partial}{\partial \llbracket \boldsymbol{\varphi} \rrbracket}\left(\frac{1}{2} \overline{\boldsymbol{P}}+\{\{\boldsymbol{t}\}\}\right): \frac{\partial \llbracket \boldsymbol{} \boldsymbol{\partial} \rrbracket}{\partial \boldsymbol{\varphi}_{+}^{J}}\right] \mathrm{d} A \\
& =\int_{\mathcal{I}_{0}} \overline{\operatorname{Grad}} \bar{N}^{I}=\left[\frac{1}{2} \frac{\partial \overline{\boldsymbol{P}}}{\partial \overline{\boldsymbol{F}}}+\frac{\partial\{\{\boldsymbol{t}\}\}}{\partial \overline{\boldsymbol{F}}}\right] \cdot \overline{\operatorname{Grad}} \bar{N}^{J}+\overline{\operatorname{Grad}}_{\bar{N}}^{I}=\left[\frac{1}{2} \frac{\partial \overline{\boldsymbol{P}}}{\partial \llbracket \boldsymbol{} \llbracket}+\frac{\partial\{\{\boldsymbol{t}\}\}}{\partial \llbracket \varphi \rrbracket}\right] \bar{N}^{J} \mathrm{~d} A,
\end{aligned}
$$

in which we have used the relation

$$
\frac{\partial \overline{\boldsymbol{F}}}{\partial \boldsymbol{\varphi}_{+}^{J}}=\frac{\partial \overline{\boldsymbol{F}}}{\partial \overline{\boldsymbol{\varphi}}^{J}} \cdot \frac{\partial \overline{\boldsymbol{\varphi}}^{J}}{\partial \boldsymbol{\varphi}_{+}^{J}}=\frac{1}{2} \frac{\partial \overline{\boldsymbol{F}}}{\partial \overline{\boldsymbol{\varphi}}^{J}} \cdot \boldsymbol{I}=\frac{1}{2} \frac{\partial}{\partial \overline{\boldsymbol{\varphi}}^{J}}\left(\overline{\boldsymbol{\varphi}}^{s} \otimes \overline{\operatorname{Grad}}^{s}\right)=\delta_{s J} \boldsymbol{I} \bar{\otimes} \overline{\operatorname{Grad}}_{N^{s}}^{s}=\boldsymbol{I} \bar{\otimes} \overline{\operatorname{Grad}} \bar{N}^{J} .
$$




\section{Appendix E. Further discussions on the choice of the material model}

Here, we elaborate on the choice of the specific free energy (58) for the interface. A basic model to capture the behavior of the interface is the isotropic interface model for the in-plane response and a cohesive type model for the orthogonal response. In order to propose a physically sound interface model, one needs to guarantee that (i) the interface free energy is invariant with respect to rigid body motions and (ii) the interface free energy satisfies certain (poly)convexity conditions. Following the representation theorem for isotropic functions, we choose the interface free energy to be a function of invariants of the interface right Cauchy-Green tensor $\overline{\boldsymbol{C}}$ and also the norm of the motion jump across the interface $\|\llbracket \varphi \rrbracket\|$. Clearly, the interface right Cauchy-Green tensor $\bar{C}$ captures the in-plane response of the interface while $\llbracket \varphi \rrbracket$ recovers the orthogonal response. Thus, the interface behavior shall be expressed as $\bar{\psi}_{0}\left(\bar{I}_{1}, \bar{I}_{2}, \bar{I}_{3}\right)$ with $\bar{I}_{1}=\overline{\boldsymbol{C}}: \overline{\boldsymbol{I}}, \bar{I}_{2}=\overline{\operatorname{Det}} \overline{\boldsymbol{C}}$ and $\bar{I}_{3}=\|\llbracket \boldsymbol{\varphi} \rrbracket\|$ being the invariants of $\overline{\boldsymbol{C}}$ and $\llbracket \boldsymbol{\varphi} \rrbracket$, respectively. An interesting consequence of isotropic interface response is that the interface stress and traction, without loss of generality, simplify to

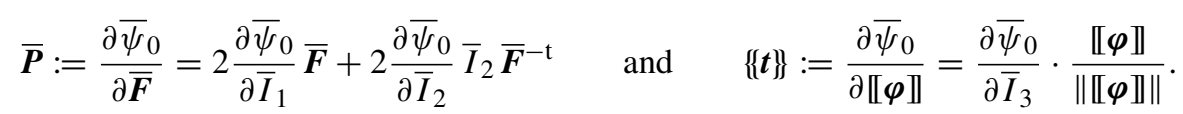

Furthermore, we assume that the interface behavior can be decomposed into its tangential and normal behavior as

$$
\bar{\psi}_{0}(\overline{\boldsymbol{F}}, \llbracket \varphi \rrbracket)=\bar{\psi}_{0}^{\|}(\overline{\boldsymbol{F}})+\bar{\psi}_{0}^{\perp}(\llbracket \varphi \rrbracket),
$$

which, in turn, can be recast into a function of invariants as

$$
\bar{\psi}_{0}\left(\bar{I}_{1}, \bar{I}_{2}, \bar{I}_{3}\right)=\bar{\psi}_{0}\left(\bar{I}_{1}, \bar{I}_{2}\right)+\bar{\psi}_{0}^{\perp}\left(\bar{I}_{3}\right) \text {. }
$$

In order to a priori satisfy the relevant (poly)convexity conditions for the interface and to fulfill the corresponding growth conditions on the interface, we choose the specific free energy

$$
\bar{\psi}_{0}\left(\bar{I}_{1}, \bar{I}_{2}, \bar{I}_{3}\right)=\frac{1}{2} \bar{\mu}\left[\bar{I}_{1}-\overline{\mathrm{PD}}-2 \ln \sqrt{\bar{I}_{2}}\right]+\frac{1}{2} \bar{\lambda}\left[\frac{1}{2}\left[\bar{I}_{2}-1\right]-\ln \sqrt{\bar{I}_{2}}\right]+\frac{1}{2} \bar{k} \bar{I}_{3}^{2},
$$

in which $\bar{\mu}, \bar{\lambda}$ and $\bar{k}$ are the interface material parameters and $\overline{\mathrm{PD}}$ stands for the interface problem dimension. In a threedimensional setting, the interface is a two-dimensional manifold and in a two-dimensional setting a one-dimensional manifold and therefore, $\overline{\mathrm{PD}}=\mathrm{PD}-1$. The free energy (E.4) is precisely the same as the interface free energy (58).

\section{References}

[1] R. Hill, Elastic properties of reinforced solids: Some theoretical principles, J. Mech. Phys. Solids 11 (1963) 357-372.

[2] R. Hill, On constitutive macro-variables for heterogeneous solids at finite strain, Proc. R. Soc. Lond. Ser. A Math. Phys. Eng. Sci. 326 (1972) $131-147$

[3] N. Daher, G.A. Maugin, The method of virtual power in continuum mechanics application to media presenting singular surfaces and interfaces, Acta Mech. 60 (1986) 217-240.

[4] G.I. Barenblatt, The formation of equilibrium cracks during brittle fracture. General ideas and hypotheses. Axially-symmetric cracks, J. Appl. Math. Mech. 23 (1959) 622-636.

[5] G.I. Barenblatt, The mathematical theory of equilibrium cracks in brittle fracture, Adv. Appl. Mech. 7 (1962) 55-129.

[6] D. Dugdale, Yielding of steel sheets containing slits, J. Mech. Phys. Solids 8 (1960) 100-104.

[7] A. Needleman, A continuum model for void nucleation by inclusion debonding, J. Appl. Mech. 54 (1987) 525-531.

[8] X.-P. Xu, A. Needleman, Numerical simulations of fast crack growth in brittle solids, J. Mech. Phys. Solids 42 (1994) 1397-1434.

[9] M. Ortiz, A. Pandolfi, Finite-deformation irreversible cohesive elements for three-dimensional crack-propagation analysis, Internat. J. Numer. Methods Engrg. 44 (1999) 1267-1282.

[10] M. Charlotte, J. Laverne, J.J. Marigo, Initiation of cracks with cohesive force models: a variational approach, Eur. J. Mech. A Solids 25 (2006) 649-669.

[11] G. Alfano, M.A. Crisfield, Finite element interface models for the delamination analysis of laminated composites: mechanical and computational issues, Internat. J. Numer. Methods Engrg. 50 (2001) 1701-1736.

[12] T.C. Gasser, G.A. Holzapfel, Geometrically non-linear and consistently linearized embedded strong discontinuity models for 3D problems with an application to the dissection analysis of soft biological tissues, Comput. Methods Appl. Mech. Engrg. 192 (2003) $5059-5098$.

[13] M.J. van den Bosch, P.J.G. Schreurs, M.G.D. Geers, An improved description of the exponential Xu and Needleman cohesive zone law for mixed-mode decohesion, Eng. Fract. Mech. 73 (2006) 1220-1234.

[14] M. Fagerström, R. Larsson, Theory and numerics for finite deformation fracture modelling using strong discontinuities, Internat. J. Numer. Methods Engrg. 66 (2006) 911-948. 
[15] K. Park, G.H. Paulino, J.R. Roesler, A unified potential-based cohesive model of mixed-mode fracture, J. Mech. Phys. Solids 57 (2009) 891-908.

[16] J. Mosler, I. Scheider, A thermodynamically and variationally consistent class of damage-type cohesive models, J. Mech. Phys. Solids 59 (2011) 1647-1668.

[17] K. Park, G.H. Paulino, Cohesive zone models: A critical review of traction-separation relationships across fracture surfaces, Appl. Mech. Rev. 64 (2013) 20.

[18] R. Dimitri, M. Trullo, L. De Lorenzis, G. Zavarise, Coupled cohesive zone models for mixed-mode fracture: A comparative study, Eng. Fract. Mech. 148 (2015) 145-179.

[19] A.I. Murdoch, A thermodynamical theory of elastic material interfaces, Quart. J. Mech. Appl. Math. 29 (1976) 245-275.

[20] G.P. Moeckel, Thermodynamics of an interface, Arch. Ration. Mech. Anal. 57 (1975) 255-280.

[21] F. dell'Isola, A. Romano, On the derivation of thermomechanical balance equations for continuous systems with a nonmaterial interface, Internat. J. Engrg. Sci. 25 (1987) 1459-1468.

[22] E. Fried, M.E. Gurtin, Thermomechanics of the interface between a body and its environment, Contin. Mech. Thermodyn. 19 (2007) $253-271$.

[23] M.E. Gurtin, A. Ian Murdoch, A continuum theory of elastic material surfaces, Arch. Ration. Mech. Anal. 57 (1975) $291-323$.

[24] D.J. Steigmann, R.W. Ogden, Elastic surface-substrate interactions, Proc. R. Soc. Lond. Ser. A Math. Phys. Eng. Sci. 455 (1999) $437-474$.

[25] E. Fried, R.E. Todres, Mind the gap: The shape of the free surface of a rubber-like material in proximity to a rigid contactor, J. Elasticity 80 (2005) 97-151.

[26] P. Chhapadia, P. Mohammadi, P. Sharma, Curvature-dependent surface energy and implications for nanostructures, J. Mech. Phys. Solids 59 (2011) 2103-2115.

[27] T. Chen, M.S. Chiu, C.N. Weng, Derivation of the generalized Young-Laplace equation of curved interfaces in nanoscaled solids, J. Appl. Phys. 100 (2006) 074308.

[28] A. Javili, A. Mcbride, P. Steinmann, Thermomechanics of solids with lower-dimensional energetics: On the importance of surface, interface, and curve structures at the nanoscale. A unifying review, Appl. Mech. Rev. 65 (2013) 010802.

[29] R. Dingreville, A. Hallil, S. Berbenni, From coherent to incoherent mismatched interfaces: A generalized continuum formulation of surface stresses, J. Mech. Phys. Solids 72 (2014) 40-60.

[30] P. Bövik, On the modelling of thin interface layers in elastic and acoustic scattering problems, Quart. J. Mech. Appl. Math. 47 (1994) $17-42$.

[31] Z. Hashin, Thin interphase/imperfect interface in elasticity with application to coated fiber composites, J. Mech. Phys. Solids 50 (2002) $2509-2537$.

[32] Y. Benveniste, T. Miloh, Imperfect soft and stiff interfaces in two-dimensional elasticity, Mech. Mater. 33 (2001) 309-323.

[33] V. Monchiet, G. Bonnet, Interfacial models in viscoplastic composites materials, Internat. J. Engrg. Sci. 48 (2010) $1762-1768$.

[34] Y. Benveniste, Models of thin interphases with variable moduli in plane-strain elasticity, Math. Mech. Solids 18 (2013) $119-134$.

[35] N.S. Ottosen, M. Ristinmaa, J. Mosler, Framework for non-coherent interface models at finite displacement jumps and finite strains, J. Mech. Phys. Solids 90 (2016) 124-141.

[36] P. Steinmann, O. Häsner, On material interfaces in thermomechanical solids, Arch. Appl. Mech. 75 (2005) 31-41.

[37] S.T. Gu, E. Monteiro, Q.C. He, Coordinate-free derivation and weak formulation of a general imperfect interface model for thermal conduction in composites, Compos. Sci. Technol. 71 (2011) 1209-1216.

[38] A. Javili, S. Kaessmair, P. Steinmann, General imperfect interfaces, Comput. Methods Appl. Mech. Engrg. 275 (2014) $76-97$.

[39] Z. Hashin, Thin interphase/imperfect interface in conduction, J. Appl. Phys. 89 (2001) 2261-2267.

[40] E. Sanchez-Palencia, Comportements local et macroscopique d'un type de milieux physiques heterogenes, Internat. J. Engrg. Sci. 12 (1974) $331-351$

[41] R. Ogden, On the overall moduli of non-linear elastic composite materials, J. Mech. Phys. Solids 22 (1974) 541-553.

[42] J.D. Eshelby, The determination of the elastic field of an ellipsoidal inclusion, and related problems, Proc. R. Soc. Lond. Ser. A Math. Phys. Eng. Sci. 241 (1957) 376-396.

[43] Z. Hashin, The moduli of an elastic solid containing spherical particles of another elastic material, in: Proceedings of I.U.T.A.M. Symposium on Non-Homogeneity in Elasticity and Plasticity, 1959, pp. 463-478.

[44] Z. Hashin, S. Shtrikman, A variational approach to the theory of the elastic behaviour of multiphase materials, J. Mech. Phys. Solids 11 (1963) $127-140$.

[45] T. Mori, K. Tanaka, Average stress in matrix and average elastic energy of materials with misfitting inclusions, Acta Metall. 21 (1973) $571-574$.

[46] R. Christensen, K. Lo, Solutions for effective shear properties in three phase sphere and cylinder models, J. Mech. Phys. Solids 27 (1979) $315-330$.

[47] M. Hori, S. Nemat-Nasser, Double-inclusion model and overall moduli of multi-phase composites, Mech. Mater. 14 (1993) $189-206$.

[48] Z. Hashin, Thermoelastic properties of fiber composites with imperfect interface, Mech. Mater. 8 (1990) 333-348.

[49] P. Ponte Castañeda, The effective mechanical properties of nonlinear isotropic composites, J. Mech. Phys. Solids 39 (1991) $45-71$.

[50] T. Mura, H.M. Shodja, Y. Hirose, Inclusion problems, Appl. Mech. Rev. 49 (1996) 118-127.

[51] W.J. Drugan, J.R. Willis, A micromechanics-based nonlocal constitutive equation and estimates of representative volume element size for elastic composites, J. Mech. Phys. Solids 44 (1996) 497-524.

[52] P.M. Suquet, Elements of homogenization theory for inelastic solid mechanics, homogenization techniques for composite media (1987) 194-278.

[53] S. Ghosh, K. Lee, S. Moorthy, Multiple scale analysis of heterogeneous elastic structures using homogenization theory and voronoi cell finite element method, Int. J. Solids Struct. 32 (1995) 27-62.

[54] H. Moulinec, P. Suquet, A numerical method for computing the overall response of nonlinear composites with complex micro-structure, Comput. Methods Appl. Mech. Engrg. 157 (1998) 69-94. 
[55] F. Feyel, Multiscale FE 2 elastoviscoplastic analysis of composite structures, Comput. Mater. Sci. 16 (1999) $344-354$.

[56] T. Kanit, S. Forest, I. Galliet, V. Mounoury, D. Jeulin, Determination of the size of the representative volume element for random composites: Statistical and numerical approach, Int. J. Solids Struct. 40 (2003) 3647-3679.

[57] M. Ostoja-Starzewski, Material spatial randomness: From statistical to representative volume element, Probab. Eng. Mech. 21 (2006) 112-132.

[58] F. Larsson, K. Runesson, S. Saroukhani, R. Vafadari, Computational homogenization based on a weak format of micro-periodicity for RVE-problems, Comput. Methods Appl. Mech. Engrg. 200 (2011) 11-26.

[59] A. Gusev, Representative volume element size for elastic composites: A numerical study, J. Mech. Phys. Solids 45 (1997) 1449-1459.

[60] T.I. Zohdi, P. Wriggers, Computational micro-macro material testing, Arch. Comput. Methods Eng. 8 (2001) 131-228.

[61] X. Chen, J.W. Hutchinson, A family of herringbone patterns in thin films, Scr. Mater. 50 (2004) 797-801.

[62] C. Miehe, J. Schotte, J. Schröder, Computational micro-macro transitions and overall moduli in the analysis of polycrystals at large strains, Comput. Mater. Sci. 16 (1999) 372-382.

[63] C. Miehe, Computational micro-to-macro transitions for discretized micro-structures of heterogeneous materials at finite strains based on the minimization of averaged incremental energy, Comput. Methods Appl. Mech. Engrg. 192 (2003) 559-591.

[64] J. Yvonnet, Q.C. He, The reduced model multiscale method (R3M) for the non-linear homogenization of hyperelastic media at finite strains, J. Comput. Phys. 223 (2007) 341-368.

[65] I. Temizer, P. Wriggers, Homogenization in finite thermoelasticity, J. Mech. Phys. Solids 59 (2011) 344-372.

[66] A. Javili, G. Chatzigeorgiou, P. Steinmann, Computational homogenization in magneto-mechanics, Int. J. Solids Struct. 50 (2013) 4197-4216.

[67] D.M. Kochmann, G.N. Venturini, Homogenized mechanical properties of auxetic composite materials in finite-strain elasticity, Smart Mater. Struct. 22 (2013) 084004.

[68] A. Javili, S. Saeb, P. Steinmann, Aspects of implementing constant traction boundary conditions in computational homogenization via semi-Dirichlet boundary conditions, Comput. Mech. http://dx.doi.org/10.1007/s00466-016-1333-8.

[69] P. Kanouté, D.P. Boso, J.L. Chaboche, B.A. Schrefler, Multiscale methods for composites: A review, Arch. Comput. Methods Eng. 16 (2009) 31-75.

[70] M.G.D. Geers, V.G. Kouznetsova, W.A.M. Brekelmans, Multi-scale computational homogenization: Trends and challenges, J. Comput. Appl. Math. 234 (2010) 2175-2182.

[71] M. Ostoja-Starzewski, S. Kale, P. Karimi, A. Malyarenko, B. Raghavan, S.I. Ranganathan, J. Zhang, Scaling to RVE in random media, Advances in Applied Mechanics 49 (2016) 111-211.

[72] S. Saeb, A. Javili, P. Steinmann, Aspects of computational homogenization at finite deformations. A unifying review from Reuss' to Voigt's bound, Appl. Mech. Rev. 68 (2016) 050801.

[73] K. Matous, M.G.D. Geers, V.G. Kouznetsova, A. Gillman, A review of predictive nonlinear theories for multiscale modeling of heterogeneous materials, J. Comput. Phys. 330 (2017) 192-220.

[74] V. Kouznetsova, M.G.D. Geers, W.A.M. Brekelmans, Multi-scale constitutive modelling of heterogeneous materials with a gradientenhanced computational homogenization scheme, Internat. J. Numer. Methods Engrg. 54 (2002) 1235-1260.

[75] R. Toupin, Elastic materials with couple-stresses, Arch. Ration. Mech. Anal. 11 (1962) 385-414.

[76] R. Mindlin, Second gradient of strain and surface-tension in linear elasticity, Int. J. Solids Struct. 1 (1965) 417-438.

[77] A.S.J. Suiker, C.S. Chang, Application of higher-order tensor theory for formulating enhanced continuum models, Acta Mech. 142 (2000) 223-234.

[78] P. Neff, J. Jeong, H. Ramezani, Subgrid interaction and micro-randomness-Novel invariance requirements in infinitesimal gradient elasticity, Int. J. Solids Struct. 46 (2009) 4261-4276.

[79] B. Svendsen, P. Neff, A. Menzel, On constitutive and configurational aspects of models for gradient continua with microstructure, ZAMM Z. Angew. Math. Mech. 89 (2009) 687-697.

[80] S. Forest, E.C. Aifantis, Some links between recent gradient thermo-elasto-plasticity theories and the thermomechanics of generalized continua, Int. J. Solids Struct. 47 (2010) 3367-3376.

[81] F. Dell'Isola, D. Steigmann, A two-dimensional gradient-elasticity theory for woven fabrics, J. Elasticity 118 (2014) $113-125$.

[82] S. Rudraraju, A.V. Ven, K. Garikipati, Three-dimensional iso-geometric solutions to general boundary value problems of Toupin's gradient elasticity theory at finite strains, Comput. Methods Appl. Mech. Engrg. 278 (2014) 705-728.

[83] A. Bertram, Finite gradient elasticity and plasticity: a constitutive mechanical framework, Contin. Mech. Thermodyn. 27 (2015) 1039-1058.

[84] A. Carcaterra, F. Dell'Isola, R. Esposito, M. Pulvirenti, Macroscopic description of microscopically strongly inhomogenous systems: A mathematical basis for the synthesis of higher gradients metamaterials, Arch. Ration. Mech. Anal. 218 (2015) 1239-1262.

[85] N. Auffray, F. Dell'Isola, V.A. Eremeyev, A. Madeo, G. Rosi, Analytical continuum mechanics á la Hamilton-Piola least action principle for second gradient continua and capillary fluids, Math. Mech. Solids 20 (2015) 375-417.

[86] F. Dell'Isola, I. Giorgio, M. Pawlikowski, N.L. Rizzi, Large deformations of planar extensible beams and pantographic lattices: heuristic homogenization, experimental and numerical examples of equilibrium, Proc. R. Soc. Lond. Ser. A Math. Phys. Eng. Sci. 472 (2016) 20150790.

[87] U. Andreaus, F. Dell'Isola, I. Giorgio, L. Placidi, T. Lekszycki, N.L. Rizzi, Numerical simulations of classical problems in two-dimensional (non) linear second gradient elasticity, Internat. J. Engrg. Sci. 108 (2016) 34-50.

[88] P. Sharma, S. Ganti, N. Bhate, Effect of surfaces on the size-dependent elastic state of nano-inhomogeneities, Appl. Phys. Lett. 82 (2003) $535-537$.

[89] H.L. Duan, J. Wang, B.L. Karihaloo, Theory of elasticity at the nanoscale, Adv. Appl. Mech. 42 (2009) 1-68.

[90] G. Chatzigeorgiou, A. Javili, P. Steinmann, Multiscale modelling for composites with energetic interfaces at the micro- or nanoscale, Math. Mech. Solids 20 (2015) 1130-1145. 
[91] J. Yvonnet, H.L. Quang, Q.C. He, An XFEM/level set approach to modelling surface/interface effects and to computing the size-dependent effective properties of nanocomposites, Comput. Mech. 42 (2008) 119-131.

[92] A. Javili, A. Mcbride, J. Mergheim, P. Steinmann, U. Schmidt, Micro-to-macro transitions for continua with surface structure at the microscale, Int. J. Solids Struct. 50 (2013) 2561-2572.

[93] A. Javili, G. Chatzigeorgiou, A.T. McBride, P. Steinmann, C. Linder, Computational homogenization of nano-materials accounting for size effects via surface elasticity, GAMM-Mitt. 38 (2015) 285-312.

[94] A. Mitrushchenkov, G. Chambaud, J. Yvonnet, Q.-C. He, Towards an elastic model of wurtzite AlN nanowires, Nanotechnology 21 (2010) 255702.

[95] H.S. Park, P.A. Klein, G. Wagner, A surface Cauchy-Born model for nanoscale materials, Internat. J. Numer. Methods Engrg. 68 (2006) 1072-1095.

[96] H.S. Park, P.A. Klein, Surface Cauchy-Born analysis of surface stress effects on metallic nanowires, Phys. Rev. B 75 (2007) 085408.

[97] D. Davydov, A. Javili, P. Steinmann, On molecular statics and surface-enhanced continuum modeling of nano-structures, Comput. Mater. Sci. 69 (2013) 510-519.

[98] N.M. Cordero, S. Forest, E.P. Busso, Second strain gradient elasticity of nano-objects, J. Mech. Phys. Solids 97 (2016) $92-124$.

[99] F. Fritzen, M. Leuschner, Nonlinear reduced order homogenization of materials including cohesive interfaces, Comput. Mech. 56 (2015) $131-151$.

[100] W. Tu, M.-J. Pindera, Cohesive zone-based damage evolution in periodic materials via finite-volume homogenization, J. Appl. Mech. 81 (2014) 101005.

[101] Z.F. Khisaeva, M. Ostoja-Starzewski, On the size of RVE in finite elasticity of random composites, J. Elasticity 85 (2006) $153-173$.

[102] J.E. Marsden, T.J. Hughes, Mathematical Foundations of Elasticity, Dover, 1994.

[103] G.A. Holzapfel, Nonlinear Solid Mechanics: A Continuum Approach for Engineering, John Wiley \& Sons, 2000.

[104] M.E. Gurtin, E. Fried, L. Anand, The Mechanics and Thermodynamics of Continua, Cambridge University Press, 2009.

[105] M.E. Gurtin, Configurational Forces as Basic Concepts of Continuum Physics, Springer, 2000.

[106] A. Javili, F. Dell'Isola, P. Steinmann, Geometrically nonlinear higher-gradient elasticity with energetic boundaries, J. Mech. Phys. Solids 61 (2013) 2381-2401.

[107] B.G. Vossen, P.J.D. Schreurs, O. van der Sluis, M.G.D. Geers, On the lack of rotational equilibrium in cohesive zone elements, Comput. Methods Appl. Mech. Engrg. 254 (2013) 146-153.

[108] N.S. Ottosen, M. Ristinmaa, J. Mosler, Fundamental physical principles and cohesive zone models at finite displacements- limitations and possibilities, Int. J. Solids Struct. 53 (2015) 70-79.

[109] S. Angenent, M.E. Gurtin, Multiphase thermomechanics with interfacial structure 2. Evolution of an isothermal interface, Arch. Ration. Mech. Anal. 108 (1989) 323-391.

[110] M.E. Gurtin, A. Struthers, Multiphase thermomechanics with interfacial structure-3. Evolving phase boundaries in the presence of bulk deformation, Arch. Ration. Mech. Anal. 112 (1990) 97-160.

[111] N.K. Simha, K. Bhattacharya, Kinetics of phase boundaries with edges and junctions in a three-dimensional multi-phase body, J. Mech. Phys. Solids 48 (2000) 2619-2641.

[112] P. Steinmann, On boundary potential energies in deformational and configurational mechanics, J. Mech. Phys. Solids 56 (2008) $772-800$.

[113] L. Mullins, Effect of stretching on the properties of rubber, Rubber Chem. Technol. 21 (1948) 281-300.

[114] R.W. Ogden, D.G. Roxburgh, A pseudo-elastic model for the Mullins effect in filled rubber, Proc. R. Soc. Lond. Ser. A Math. Phys. Eng. Sci. 455 (1999) 2861-2877.

[115] A. Dorfmann, R.W. Ogden, A constitutive model for the Mullins effect with permanent set in particle-reinforced rubber, Int. J. Solids Struct. 41 (2004) 1855-1878.

[116] A. Javili, A. McBride, P. Steinmann, B.D. Reddy, A unified computational framework for bulk and surface elasticity theory: A curvilinearcoordinate-based finite element methodology, Comput. Mech. 54 (2014) 745-762.

[117] T.J. Hughes, The Finite Element Method: Linear Static and Dynamic Finite Element Analysis, Dover, 2000.

[118] P. Wriggers, Nonlinear Finite Element Methods, Springer, 2008.

[119] R.W. Ogden, Large deformation isotropic elasticity-On the correlation of theory and experiment for incompressible rubberlike solids, Proc. R. Soc. Lond. Ser. A Math. Phys. Eng. Sci. 326 (1972) 565-584.

[120] C. Linder, M. Tkachuk, C. Miehe, A micromechanically motivated diffusion-based transient network model and its incorporation into finite rubber viscoelasticity, J. Mech. Phys. Solids 59 (2011) 2134-2156.

[121] M. Tkachuk, C. Linder, The maximal advance path constraint for the homogenization of materials with random network micro-structure, Phil. Mag. 92 (2012) 2779-2808.

[122] A. Raina, C. Linder, A homogenization approach for nonwoven materials based on fiber undulations and reorientation, J. Mech. Phys. Solids 65 (2014) 12-34.

[123] V.I. Levitas, J.A. Warren, Phase field approach with anisotropic interface energy and interface stresses: Large strain formulation, J. Mech. Phys. Solids 91 (2016) 94-125.

[124] M. Ohman, F. Larsson, K. Runesson, Computational homogenization of liquid-phase sintering based on a mixed variational format, GAMMMitt. 39 (2016) 189-209.

[125] R.M. Bowen, C.C. Wang, Introduction to Vectors and Tensors: Linear and Multilinear Algebra, 1976.

[126] E. Kreyszig, Differential Geometry, Dover, 1991.

[127] P.G. Ciarlet, An Introduction to Differential Geometry with Applications to Elasticity, 2005.

[128] P. Steinmann, Geometrical Foundations of Continuum Mechanics: An Application to First- and Second-Order Elasticity and ElastoPlasticity, Springer, 2015. 